\title{
Search for physics beyond the standard model in final states with a lepton and missing transverse energy in proton-proton collisions at $\sqrt{s}=8 \mathrm{TeV}$
}

\author{
V. Khachatryan et al. \\ (CMS Collaboration) \\ (Received 12 August 2014; published 22 May 2015)
}

\begin{abstract}
A search for new physics in proton-proton collisions having final states with an electron or muon and missing transverse energy is presented. The analysis uses data collected in 2012 with the CMS detector, at an LHC center-of-mass energy of $8 \mathrm{TeV}$, and corresponding to an integrated luminosity of $19.7 \mathrm{fb}^{-1}$. No significant deviation of the transverse mass distribution of the charged lepton-neutrino system from the standard model prediction is found. Mass exclusion limits of up to $3.28 \mathrm{TeV}$ at $95 \%$ confidence level for a $W^{\prime}$-boson with the same couplings as that of the standard model $W$-boson are determined. Results are also derived in the framework of split universal extra dimensions, and exclusion limits on Kaluza-Klein $W_{\mathrm{KK}}^{(2)}$ states are found. The final state with large missing transverse energy also enables a search for dark matter production with a recoiling $W$-boson, with limits set on the mass and the production cross section of potential candidates. Finally, limits are established for a model including interference between a left-handed $W^{\prime}$-boson and the standard model $W$-boson and for a compositeness model.
\end{abstract}

DOI: 10.1103/PhysRevD.91.092005

PACS numbers: 14.70.Pw, 12.60.Cn, 13.85.Rm

\section{INTRODUCTION}

The standard model (SM) of particle physics is a theory of the structure of matter, describing the properties of all known elementary particles and the forces between them. Having been studied experimentally for five decades, its predictions have been verified with very high precision. Despite the great success of the SM, beyond the SM (BSM) physics addresses a variety of open issues. To name a few examples, the relationship of the electroweak and gravitational energy scales must be understood and incorporated in the theory, an underlying concept is needed to explain the origin of the observed three fermion families, and astrophysical observations indicate the presence of dark matter (DM) not described in the standard model. Many SM extensions predict additional heavy gauge bosons, including models with extended gauge sectors, designed to achieve gauge coupling unification, and theories with extra spatial dimensions. BSM physics can be detected through observation of significant deviations from SM predictions.

The search presented in this paper is sensitive to deviations from the SM prediction for the transverse mass spectrum of events with a charged lepton (electron or muon) and one or more particles that cannot be directly detected (neutrino, dark matter particle) in the final state. Additionally, events are allowed to include an arbitrary number of jets, as they may originate from initial state

*Full author list given at the end of the article.

Published by the American Physical Society under the terms of the Creative Commons Attribution 3.0 License. Further distribution of this work must maintain attribution to the author(s) and the published article's title, journal citation, and DOI. radiation. Interpretations of the observations are made in the context of various theoretical models: the sequential standard model (SSM) with a $W^{\prime}$-boson [1], a helicitynonconserving contact interaction model (HNC-CI) [2], a DM model with a DM particle recoiling against a $W$-boson [3-5], namely the sequential standard model with same-sign couplings (SSMS) and the sequential standard model with opposite-sign couplings (SSMO) [6-8], split universal extra dimensions (split-UED) $[9,10]$, and $\mathrm{a} \mathrm{TeV}^{-1}$ model [11-14], the latter two predicting an additional spatial dimension.

Since the discovery of the $W$-boson, experiments have scrutinized the lepton and missing transverse energy channel for evidence of physics beyond the standard model. Neither searches by the Tevatron experiments D0 [15] and CDF [16] nor searches carried out previously at the LHC experiments ATLAS [17-20] and CMS [21-24] have found any indication for such a deviation. The present analysis improves upon the discovery potential of its predecessors. It is based on data from an integrated luminosity of $19.7 \pm$ $0.5 \mathrm{fb}^{-1}$ [25] of proton-proton collisions at a center-of mass energy of $8 \mathrm{TeV}$, recorded in 2012 with the CMS detector [26] at the CERN LHC.

The search for new physics is carried out in the transverse invariant mass distribution. The shape of the distribution is taken into account by using a binned likelihood method. This approach is especially important as the examined theories predict very different signal event distributions. While the SSM $W^{\prime}$-boson can be discovered at very high transverse mass, the DM and CI models manifest themselves as event excesses at lower values of the transverse mass. A $W^{\prime}$-boson interfering with the standard model $W$-boson can even lead to a deficit of events in some regions compared to the SM prediction. 
This paper is structured as follows. Section II describes the experimental setup. The theoretical models are explained in Sec. III. A discussion of the event reconstruction and selection criteria in Sec. IV is followed by a presentation of the transverse mass distribution of the selected events in Sec. V. In Sec. VI detailed information about the relevant background processes and their prediction is given. A thorough determination of the uncertainties (Sec. VII) is essential in order to interpret the results. Limitsetting procedures are explained in Sec. VIII. In Sec. IX the limits in terms of the different signal models are derived. A summary of the results is given in Sec. X.

\section{CMS DETECTOR}

The central feature of the CMS apparatus is a superconducting solenoid of $6 \mathrm{~m}$ internal diameter, providing a magnetic field of $3.8 \mathrm{~T}$. Within the superconducting solenoid volume are a silicon pixel and strip tracker, a lead tungstate crystal electromagnetic calorimeter (ECAL), and a brass/scintillator hadron calorimeter, each composed of a barrel and two end cap sections. Muons are measured in gas-ionization detectors embedded in the steel fluxreturn yoke outside the solenoid. Extensive forward calorimetry complements the coverage provided by the barrel and end cap detectors.

The electromagnetic calorimeter consists of 75848 lead tungstate crystals which provide coverage in pseudorapidity $|\eta|<1.479$ in a barrel region and $1.479<|\eta|<3.0$ in two end cap regions.

The ECAL energy resolution for electrons with a transverse energy $E_{\mathrm{T}} \approx 45 \mathrm{GeV}$ from $Z \rightarrow e e$ decays is better than $2 \%$ in the central region of the ECAL barrel $(|\eta|<0.8)$ and is between $2 \%$ and 5\% elsewhere [27]. For high energies, which are relevant for this analysis, the electron energy resolution slightly improves [28].

Muons are measured in the pseudorapidity range $|\eta|<2.4$, with detection planes made using three technologies: drift tubes, cathode strip chambers, and resistive plate chambers. Matching muons to tracks measured in the silicon tracker results in a relative transverse momentum resolution in the barrel of about $1 \%$ for muons with a transverse momentum $p_{\mathrm{T}}$ of up to about $200 \mathrm{GeV}$ and better than $10 \%$ for high momentum muons of $p_{\mathrm{T}} \sim 1 \mathrm{TeV}$ [29].

A more detailed description of the CMS detector, together with a definition of the coordinate system used and the relevant kinematic variables, can be found in Ref. [26].

\section{PHYSICS MODELS AND SIGNAL SIMULATION}

Many models of new physics predict $W$-boson-like particles decaying with an experimental signature of a charged lepton $\ell$ and missing transverse energy $E_{\mathrm{T}}^{\text {miss }}$, which may flag the presence of a noninteracting particle.
$\vec{p}_{\mathrm{T}}^{\text {miss }}$ is defined as $-\sum \vec{p}_{\mathrm{T}}$ of all reconstructed particles with $E_{\mathrm{T}}^{\text {miss }}$ being the modulus of $\vec{p}_{\mathrm{T}}^{\text {miss }}$. These additional heavy vector bosons may arise in models with more symmetry groups, extra dimensions, compositeness, or other scenarios. Their presence may be detected as a feature in the observed spectrum of transverse mass, defined as

$$
M_{\mathrm{T}}=\sqrt{2 p_{\mathrm{T}}^{\ell} E_{\mathrm{T}}^{\mathrm{miss}}\left(1-\cos \left[\Delta \phi\left(\ell, \vec{p}_{\mathrm{T}}^{\mathrm{miss}}\right)\right]\right)},
$$

where $\Delta \phi\left(\ell, \vec{p}_{\mathrm{T}}^{\text {miss }}\right)$ is the azimuthal opening angle between the directions of the missing transverse energy and that of the charged lepton. The spectrum is expected to be dominated by the $W$-boson background, which has a very small cross section at high $M_{\mathrm{T}}$. Most new physics models predict high- $p_{\mathrm{T}}$ leptons, which should be identifiable in the low-background region.

This section summarizes the new physics models used for interpretation of the observations, along with modelspecific assumptions and details of the generator programs used for production of simulated signal event samples. All generated signal events are processed through a full simulation of the CMS detector based on GEANT4 [30,31], a trigger emulation, and the event reconstruction chain. An overview of the models considered is given in Table I. Three representative signal examples (sequential standard model, contact interactions, and dark matter) are used as examples in the distributions throughout the paper. Diagrams of these three signals are shown in Fig. 1. In addition, specific model variations are discussed: the sequential standard model with $W-W^{\prime}$ interference (SSMO and SSMS) and two models with one extra dimension and selective particle couplings to this dimension. All limits are given at 95\% C.L. unless stated otherwise.

The analysis is performed in two channels, the $e+E_{\mathrm{T}}^{\text {miss }}$ and the $\mu+E_{\mathrm{T}}^{\text {miss }}$ channel, where the charged lepton is required to be prompt. Final states where the electron or muon originates from, e.g., a $\tau$ decay, are not considered as a signal. Therefore, the results can be interpreted for each coupling individually. Only the dark matter model is exempt from that rule. In this model the lepton is produced via a standard model interaction as shown in Fig. 1, and thus events with nonprompt leptons originating from $\tau$ decays are also considered.

\section{A. Sequential standard model $W^{\prime}$-boson}

In the SSM, the $W^{\prime}$-boson, as shown in Fig. 1 (left), is considered to be a heavy analog of the SM $W$-boson, with similar decay modes and branching fractions. These are modified by the presence of the $t \bar{b}$ decay channel, which opens up for $W^{\prime}$-boson masses above $180 \mathrm{GeV}$. Dedicated searches in this channel are described in Refs. [32-37]. This analysis considers $W^{\prime}$-boson masses of $\geq 300 \mathrm{GeV}$, yielding a predicted branching fraction $(\mathcal{B})$ of about $8.5 \%$ 
TABLE I. Overview of models considered, with the relevant model parameters. The models are explained in the text with more detail.

\begin{tabular}{|c|c|c|}
\hline Model name & Parameters & Description \\
\hline $\begin{array}{l}\text { Sequential standard model } \\
\text { without interference [1] }\end{array}$ & $M_{W^{\prime}}$ & $\begin{array}{l}\text { The SSM } W^{\prime} \text {-boson does not interfere with the } W \text {-boson. It has the same coupling strength } \\
\text { to fermions as the } W \text {-boson, and its decay width is determined by its mass. }\end{array}$ \\
\hline $\begin{array}{l}\text { Helicity-non-conserving } \\
\text { contact interaction } \\
\text { model [2] }\end{array}$ & $\Lambda$ & $\begin{array}{l}\text { A four-fermion contact interaction model. Quarks and leptons are composite objects of } \\
\text { fundamental constituents. No interference effects occur in this model. }\end{array}$ \\
\hline $\begin{array}{l}\text { Dark matter effective } \\
\text { theory }[3-5]\end{array}$ & $M_{\chi}, \Lambda, \xi$ & $\begin{array}{l}\text { A dark matter model with } W \text {-boson radiation. Fermionic dark matter particles have an } \\
\text { effective coupling to quarks. An SM } W \text {-boson recoils against the pair of dark matter } \\
\text { particles. }\end{array}$ \\
\hline $\begin{array}{l}\text { Sequential standard model } \\
\text { with same sign } \\
\text { couplings }[6-8]\end{array}$ & $M_{W^{\prime}}$ & $\begin{array}{l}\text { The SSMS } W^{\prime} \text {-boson interferes with the SM } W \text {-boson and couples in the same way to } \\
\text { fermions. This leads to a destructive interference for } M_{W}<M_{\mathrm{T}}<M_{W^{\prime}} \text { and to a } \\
\text { constructive interference for } M_{\mathrm{T}}>M_{W^{\prime}} \text {. The coupling strength can vary, resulting in } \\
\text { different widths. }\end{array}$ \\
\hline $\begin{array}{l}\text { Sequential standard model } \\
\text { with opposite sign } \\
\text { couplings }\end{array}$ & $M_{W^{\prime}}$ & $\begin{array}{l}\text { Similar to SSMS, with the } W \text { - and } W^{\prime} \text {-boson couplings to quarks having the opposite sign. } \\
\text { This leads to a constructive interference for } M_{W}<M_{\mathrm{T}}<M_{W^{\prime}} \text {. The coupling strength } \\
\text { can vary, resulting in different widths. }\end{array}$ \\
\hline $\begin{array}{l}\text { Split universal extra } \\
\text { dimensions model }[9,10]\end{array}$ & $\mu, R$ & $\begin{array}{l}\text { The tower of } W_{\mathrm{KK}}^{(\mathrm{n})} \text { Kaluza-Klein excitations has the same couplings as the } W \text {-boson. Only } \\
\text { if the degree of excitation } n \text { is even, the } W_{\mathrm{KK}}^{(\mathrm{n})} \text {-boson couples to SM fermions. The LHC } \\
\text { is expected to be sensitive only to the second excitation in the tower }(n=2) \text {. The size of } \\
\text { the extra dimension } R \text { determines the mass of the } W_{\mathrm{KK}}^{(\mathrm{n})} \text {-boson. Interference with the SM } \\
W \text {-boson is not considered. }\end{array}$ \\
\hline $\begin{array}{l}\mathrm{TeV}^{-1} \text { model with a single } \\
\text { additional spatial } \\
\text { dimension }[11-14]\end{array}$ & $M_{\mathrm{C}}$ & $\begin{array}{l}\text { SM } W \text {-bosons propagate into the additional dimension as Kaluza-Klein states. Their } \\
\text { coupling constant to fermions is } \sqrt{2} \text { times larger than that of the SM } W \text {-boson. The } \\
\text { compactification scale is denoted } M_{\mathrm{C}} \text { in this model. }\end{array}$ \\
\hline
\end{tabular}

for each of the leptonic channels studied. Under these assumptions, the width of a $1 \mathrm{TeV} W^{\prime}$-boson would be about $33 \mathrm{GeV}$. Decays of the $W^{\prime}$-boson via $W Z$ are assumed to be suppressed; dedicated searches for these decays can be found in Refs. [38,39].

The SSM [1] is a benchmark model used as a reference point for experimental $W^{\prime}$-boson searches for more than two decades. The Tevatron experiments D0 and CDF established mass exclusion limits of around $1.00 \mathrm{TeV}$ [15] and $1.12 \mathrm{TeV}$ [16], respectively. The $W^{\prime}$-boson searches were among the first analyses to be performed at the LHC, exploiting the large center-of-mass energy. The LHC experiments ATLAS and CMS have more recently raised the $W^{\prime}$-boson mass exclusion to values around $2.5 \mathrm{TeV}$ [17-19,21-24].

In accordance with previous analyses [15-19,21-24], no interference with the SM $W$-boson is considered. The absence of interference can be interpreted as the result of a $\mathrm{V}+\mathrm{A}$ coupling of the SSM $W^{\prime}$-boson. The signature of a charged high-momentum lepton and missing transverse energy would be observed in the decays of such a $W^{\prime}$ boson predicted by left-right symmetric models [40-43]. This particle is typically assumed to have a heavy righthanded neutrino among its decay products [44-46]. However, the mass of the right-handed neutrino is not constrained, and it could be light as long as it does not couple to SM weak bosons. The transverse-mass signature is a Jacobian peak, similar to that of the SM $W$-boson but at much higher masses, as shown by the blue line in Fig. 3 (top left). With increasing $W^{\prime}$-boson masses, the phase space for production in $p p$ collisions at $8 \mathrm{TeV}$ decreases, because of constraints from the parton distribution functions (PDFs), leading to a growing fraction produced off shell at lower masses.

The simulation of data samples in the SSM is performed at leading order (LO) with PYTHIA 6.4.26 [47], using the
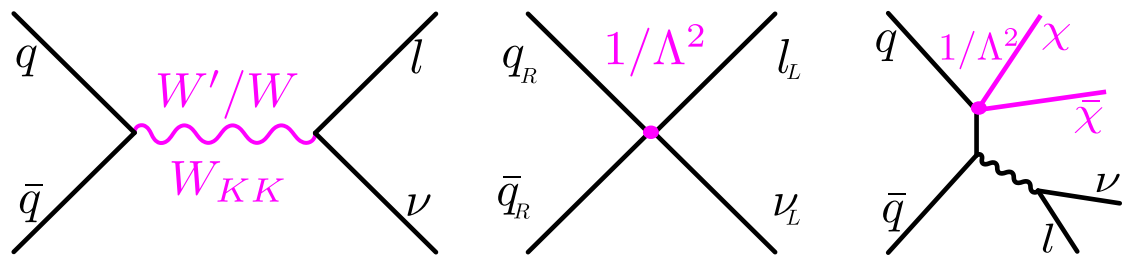

FIG. 1 (color online). Production and decay of an SSM $W^{\prime}$ or $W_{\mathrm{KK}}$-boson (left), HNC-CI (center), DM single $W$ - boson production (right). 
CTEQ6L1 PDFs [48]. A $W^{\prime}$-boson mass-dependent Kfactor is used to correct for next-to-next-to-leading-order (NNLO) QCD cross sections, calculated using FEWZ $[49,50]$. The K-factors vary from 1.363 to 1.140 .
Table II shows the LO and NNLO cross sections for this model. The NNLO corrections decrease with $W^{\prime}$-boson masses up to around $2.5 \mathrm{TeV}$. For higher masses, the Kfactor increases and becomes similar to the low-mass

TABLE II. Signal production cross sections.

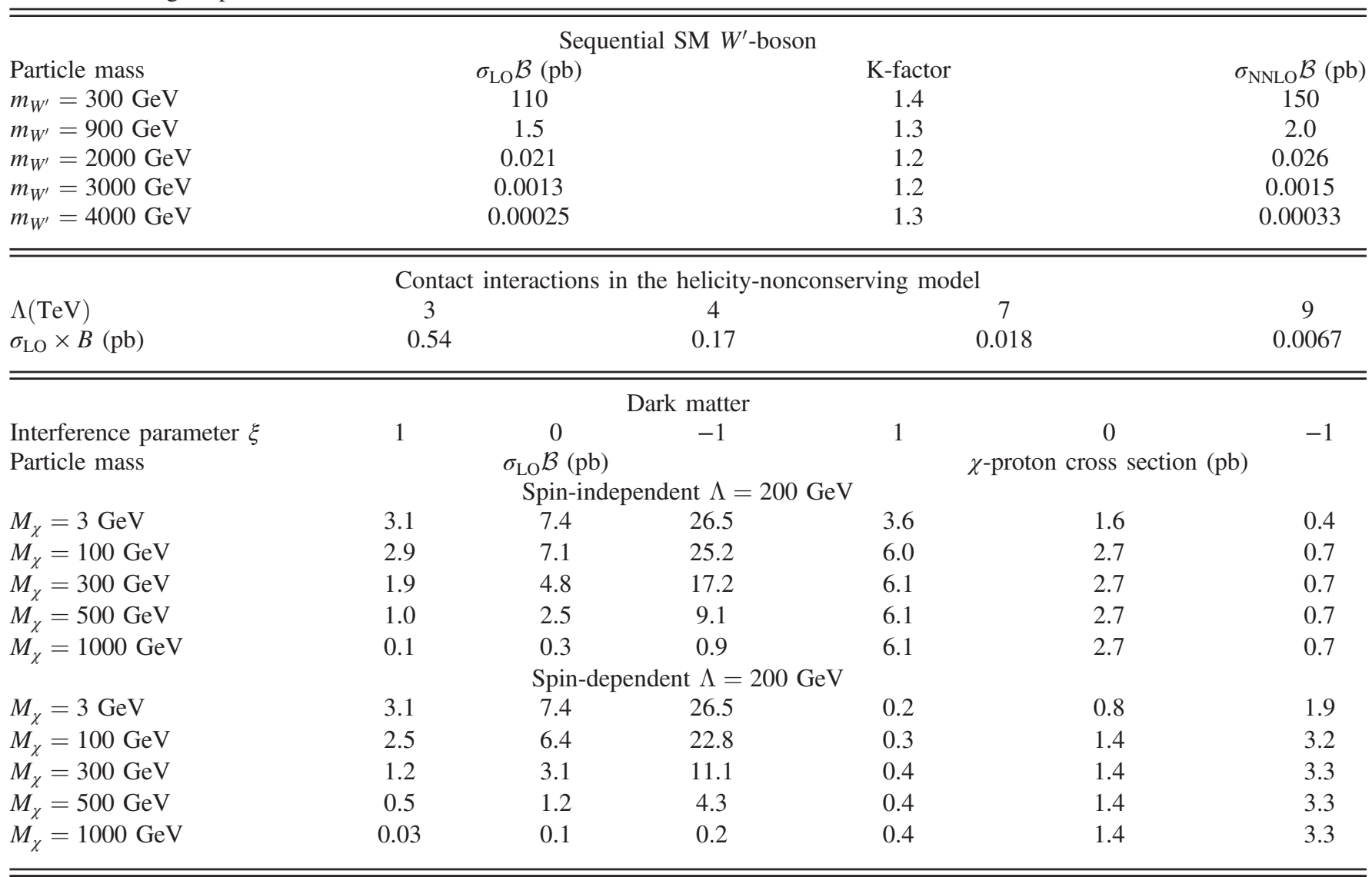

Particle mass

$m_{W^{\prime}}=300 \mathrm{GeV}$

$m_{W^{\prime}}=500 \mathrm{GeV}$

$m_{W^{\prime}}=1000 \mathrm{GeV}$

$m_{W^{\prime}}=2000 \mathrm{GeV}$

$m_{W^{\prime}}=3000 \mathrm{GeV}$

Models with interference of $W$ - and $W^{\prime}$-bosons ( $W$ - boson LO cross section subtracted)

$$
\begin{array}{cc}
\text { SSMS } \sigma_{\mathrm{LO}} \mathcal{B}(\mathrm{pb}) & \mathrm{SSMO} \sigma_{\mathrm{LO}} \mathcal{B}(\mathrm{pb}) \\
33 \pm 59 & 90 \pm 81 \\
11 \pm 58 & 21 \pm 57 \\
0.12 \pm 0.85 & 1.562 \pm 0.099 \\
-0.030 \pm 0.040 & 0.0460 \pm 0.0079 \\
-0.0064 \pm 0.0013 & 0.0125 \pm 0.0018
\end{array}
$$

\begin{tabular}{lcccc}
\hline \hline & & Split-UED $W_{\mathrm{KK}}^{(2)}$-boson & & \\
& & $\mu=0.05 \mathrm{TeV}$ & & \\
Particle mass & $\sigma_{\mathrm{LO}} \mathcal{B}(\mathrm{pb})$ & $\sigma_{\mathrm{NNLO}} \mathcal{B}(\mathrm{pb})$ & $\sigma_{\mathrm{LO}} \mathcal{B}(\mathrm{pb})$ & 250 \\
$m_{W_{\mathrm{KK}}^{(2)}}=300 \mathrm{GeV}$ & 42 & 56 & 37 & $\sigma_{\mathrm{NNLO}} \mathcal{B}(\mathrm{pb})$ \\
$m_{W_{\mathrm{KK}}^{(2)}}=500 \mathrm{GeV}$ & 2.3 & 3.1 & 2.0 & 340 \\
$m_{W_{\mathrm{KK}}^{(2)}}=1000 \mathrm{GeV}$ & 0.030 & 0.040 & 0.050 & 0.061 \\
$m_{W_{\mathrm{KK}}^{(2)}}=2000 \mathrm{GeV}$ & 0.00013 & 0.00016 & 0.0042 & 0.0048 \\
$m_{W_{\mathrm{KK}}^{(2)}}=3000 \mathrm{GeV}$ & 0.0000014 & 0.0000016 & & \\
\hline \hline
\end{tabular}

Particle mass

$\mathrm{TeV}^{-1}$ model ( $W$-boson LO cross section subtracted)

$M_{C}=2000 \mathrm{GeV}$

$\sigma_{\mathrm{LO}} \mathcal{B}(\mathrm{pb})$

$M_{C}=2600 \mathrm{GeV}$ 
values, because of the increased fraction of off-shell production (see Table II).

\section{B. Contact interactions in the helicity-nonconserving model}

Another interpretation of an enhancement in the $\ell+$ $E_{\mathrm{T}}^{\text {miss }}$ final state can be made in terms of a specific fourfermion contact interaction (CI), shown schematically in Fig. 1 (middle). The considered model assumes that quarks and leptons are composite objects with fundamental constituents [51], motivated by the observation of mass hierarchies in the fermion sector.

At energies much lower than the binding energy, denoted by $\Lambda$, the quark and lepton compositeness manifests itself as a four-fermion CI. The CI between two quarks, a neutrino, and a charged lepton is described by the helicitynonconserving (HNC) model [2]. The corresponding cross section is $\sigma_{\mathrm{CI} \rightarrow \mu \nu}=(\pi \hat{s}) /\left(12 \Lambda^{4}\right)$, where $\hat{s}$ is the center-ofmass energy of the partons. Typical cross sections for different values of $\Lambda$ are shown in Table II. In the HNC model, there is no interference of the final state with the SM $W$-boson because of the difference in the chiral structures. The $M_{\mathrm{T}}$ spectrum for CI would yield a nonresonant excess, increasing with $M_{\mathrm{T}}$ relative to the SM expectation, shown in Fig. 3 (top right). Until recently, no limit on the compositeness energy scale had been set in the muon channel in the HNC-CI model. A previous version of this analysis [24] set a limit of $10.5 \mathrm{TeV}$ and updated the previous CDF limit in the electron channel from $\Lambda=2.81 \mathrm{TeV}$ [52].

Signal samples for this model were produced with PYTHIA at LO. There are no existing higher-order calculations for this model, and LO cross sections are used.

\section{Dark matter}

One commonly used method to describe direct dark matter production at colliders is the use of an effective field theory (EFT) [3-5]. The matrix element is a four-fermion contact interaction with two quarks in the initial state and two fermionic dark matter particles $(\chi \bar{\chi})$ in the final state. This process would not result in any directly detectable final state objects. The process may be triggered and analyzed through observation of a SM $W$-boson recoiling against the dark matter, as shown in Fig. 1 (right). A search in this leptonic decay mode complements established monojet and monophoton searches [53,54] and has the advantage of lower SM background, as well as the presence of a lepton to trigger the event. This study of the singlelepton channel follows the strategy outlined in Ref. [55]. Because the $p_{\mathrm{T}}$ sum of noninteracting particles must be balanced by the charged one, we use two-body decay kinematics for the reconstruction of these events.

Under the assumption of a weakly interacting particle, different couplings are possible. In analogy with the SM weak interaction, the following two couplings are assumed: spin-independent vector coupling

$$
\frac{1}{\Lambda^{2}} \bar{\chi} \gamma^{\mu} \chi \cdot \lambda_{i} \bar{q}_{i} \gamma_{\mu} q_{i}
$$

spin-dependent axial-vector coupling

$$
\frac{1}{\Lambda^{2}} \bar{\chi} \gamma^{\mu} \gamma^{5} \chi \cdot \lambda_{i} \bar{q}_{i} \gamma_{\mu} \gamma^{5} q_{i}
$$

The model parameters are the scale of the effective interaction $\Lambda=M_{\text {messenger }} / \sqrt{g_{\mathrm{DM}}}$, which combines a heavy messenger particle with its coupling constants to dark matter and to quarks $g_{\mathrm{DM}}$. The mass of the dark matter particle is denoted $M_{\chi}$ and is included in the spinors $\chi$ and $\bar{\chi}$. The parameter $\lambda_{i}$ introduces a relative coupling strength, which in general could be different for each quark flavor.

Dark matter can couple to either up- or down-type quarks with the same initial and final state, as shown in Fig. 2. Given that the couplings to up- or down-type quarks yield similar behavior, the parameter $\xi=\lambda_{u} \lambda_{d}$ is most important for the phenomenology. Following Ref. [55], we consider three scenarios with $\left|\lambda_{i}\right|=1$ or 0 . A value of $\xi= \pm 1$ maximizes the effects of interference. A choice of $\xi=0$ can be assumed in two different ways, suppressing either the coupling to up- or to down-type quarks. Both cases are shown in Fig. 3 (middle left). The difference between the two cases is small; therefore, in the following we only consider the case with suppressed couplings to down-type quarks $\left(\lambda_{d}=0\right)$ and denote it as $\xi=0$. The choice of the interference parameter changes the total cross section and the shape of the $M_{\mathrm{T}}$ spectrum, as shown in Fig. 3 (middle left) for $\Lambda=600 \mathrm{GeV}, M_{\chi}=10 \mathrm{GeV}$.

In searches at proton-proton colliders, the difference between vector and axial-vector coupling is less important than in direct DM-nucleon interaction experiments, as can be seen from Table II. This is due to the large influence of the spin on the interaction at low $Q^{2}$ (of the order of 1 to $100 \mathrm{keV}$ [56]), which is relevant for direct detection experiments, where coherent scattering at the nucleus is only possible for spin-independent (vector) couplings but not for the spin-dependent (axial-vector) couplings. At the LHC, half of the initial quarks originate from the

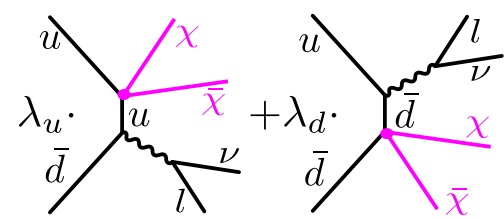

FIG. 2 (color online). Feynman diagrams for dark matter interference, shown as an example with an up and a down quark. The same initial and final state can have different particles coupling to the dark matter particles. 

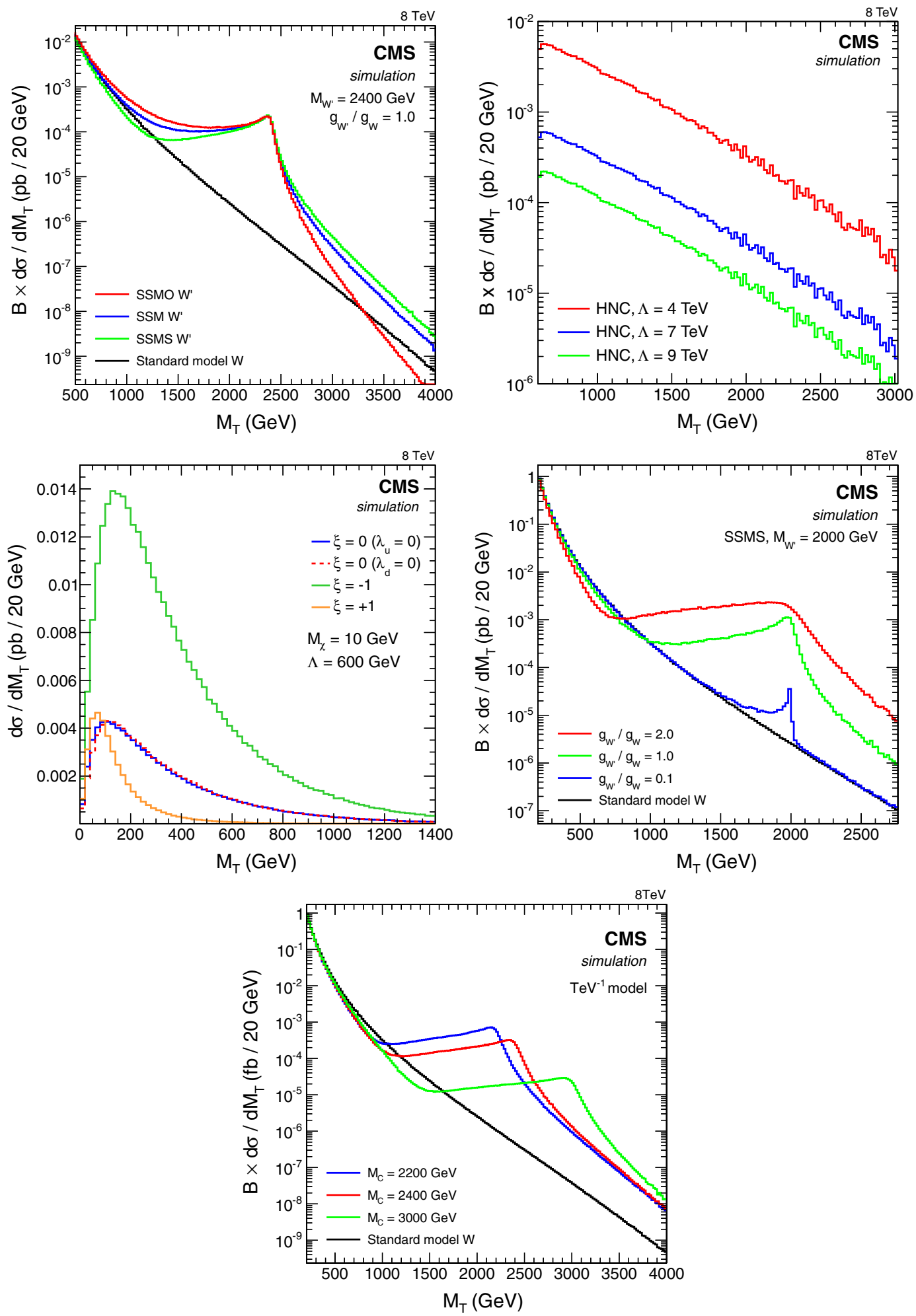

FIG. 3 (color online). Signal shapes at generator level: SSM model compared to the SSMS and SSMO models for $g_{W^{\prime}} / g_{W-}=1$ (top left), HNC-CI model for various values of $\Lambda$ (top right), DM for various values of $\xi$ (middle left), $W^{\prime}$ with $W$-boson interference and a varying coupling strength $g_{W} / g_{W^{\prime}}$ in the same sign scenario (middle right), and $\mathrm{TeV}^{-1}$ model (bottom). 
quark-gluon sea, and all spin configurations and lightquark flavors are available for production. For low $M_{\chi}$, no difference is observed between vector and axialvector couplings, as shown in Fig. 3 (middle left) for $M_{\chi}=10 \mathrm{GeV}$. For masses above $100 \mathrm{GeV}$, axial-vector cross sections are lower than the vector cross sections, without a significant shape difference.

The validity of this effective theory model is limited. For $\Lambda>M_{\chi} / 2 \pi$ the coupling is perturbative. A more stringent criterion is $g_{\mathrm{DM}}=1$, which constrains the model to $\Lambda>2 M_{\chi}$.

Simulated signal samples are produced with MADGRAPH 5.1.5 [57] matched to PYTHIA for showering and hadronization. The search is inclusive in terms of jet multiplicity, and no constraints on the number of jets are applied. The samples are simulated for $\xi=+1$ and are rescaled on an event-by-event basis for $\xi=0$ and -1 .

\section{Interference of $W$ - and $W^{\prime}$-bosons with variable coupling strength}

If the $W^{\prime}$-boson interacts with left-handed particles and right-handed antiparticles ( $\mathrm{V}-\mathrm{A}$ coupling), interference with the $W$-boson is expected [6-8]. The lowest-order effective Lagrangian for the interaction of two fermions and such a $W^{\prime}$-boson is

$$
\mathcal{L}=\frac{V^{i j}}{2 \sqrt{2}} g_{W^{\prime}}^{i j} \bar{f}^{i} \gamma^{\mu}\left(1-\gamma^{5}\right) W_{\mu}^{\prime} f^{j}+\text { H.c. },
$$

where h.c. is the Hermitian conjugate, $V_{i j}$ is the CabibboKobayashi-Maskawa matrix element for quarks and unity for leptons, and $g_{W^{\prime}}$ is the coupling constant. The nature of the interference effects depends on the ratio of the coupling $g_{W^{\prime}}$ to the SM weak coupling constant $g_{W-}=e / \sin \theta_{\mathrm{W}}$. Two different scenarios are considered: the same sign scenario with $g_{W^{\prime}}^{\ell \nu} g_{W^{\prime}}^{q q^{\prime}}>0$ and the opposite sign scenario with $g_{W^{\prime}}^{\ell \nu} g_{W^{\prime}}^{q q^{\prime}}<0$. The absolute value of the coupling $g_{W^{\prime}}$ is considered the same for quarks and leptons.

For the SSMS, the differential cross section for $W+W^{\prime}$ boson production can be smaller than the SM $W$-boson cross section, reflecting the effect of destructive interference. This effect is shown by the green curve in Fig. 3 (top left) and is discussed in Ref. [6]. In the SSMO, the cross sections exceed the SSM cross sections because of constructive interference, shown by the red curve in the same figure.

The coupling strength $g_{W^{\prime}}$ affects both the total cross section and the width of the Jacobian peak. It also influences the impact of the interference effects on the $M_{\mathrm{T}}$ spectrum. Assuming $g_{W^{\prime}}=g_{W-}$ the Jacobian peak partial widths of a $W^{\prime}$-boson decaying to leptons can be seen in Fig. 3 (top left). For a larger coupling, the width increases, as shown in Fig. 3 (middle right). This behavior is taken into account when deriving mass limits as a function of the coupling strength. A similar strategy was used in previous $W^{\prime} \rightarrow t b$ analysis [15,33].

The contributions of the $W$-and $W^{\prime}$-bosons to the overall cross section must be simulated simultaneously, as the final scattering amplitude is the sum of these two terms squared. An $M_{\mathrm{T}}$ requirement is used at event generation to reduce the $W$-boson contribution resulting from the large $W$-boson production cross section, which is several orders of magnitude larger than that for the $W^{\prime}$-boson production; $\sigma_{\mathrm{LO}}(W-\rightarrow \ell \nu)$ falls from $9600 \mathrm{pb}$ to $1.5 \mathrm{fb}$ when the requirement $M_{\mathrm{T}}>500 \mathrm{GeV}$ is imposed.

To provide an impression about the influence of the $W^{\prime}$-boson on the total cross section, effective cross sections are provided in Table II for the SSMS and SSMO models. They are derived from simulations of $W+W^{\prime}$-boson production with interference, with subsequent subtraction of the $W$-boson background. For each mass point, an individual $M_{\mathrm{T}}$ cutoff is chosen and applied to both the $W$-boson sample and the $W+W^{\prime}$ sample before subtraction. This lowers the $W$-boson contribution and therefore reduces the large uncertainty induced when subtracting two large and similar numbers. The cutoff is selected at the $M_{\mathrm{T}}$ value where the two distributions are compatible within their statistical uncertainties. For example, the cutoff for the $W^{\prime}$-boson of $2 \mathrm{TeV}$ in mass has been chosen to be $250 \mathrm{GeV}$. It is then varied up and down by $40 \mathrm{GeV}$ to represent the interval in which the two distributions approach each other and the procedure is repeated. The difference of the varied subtractions is provided as uncertainty in Table II. The resulting effective $W^{\prime}$ cross section can be compatible with 0 or even less in the case of destructive interference. These effective cross sections are meant to provide additional information for the reader and are not used in the analysis, which is based on the combined $W+W^{\prime}$-boson production cross section.

A model of a $W^{\prime}$-boson with a SM-like left-handed coupling has been implemented within the MADGRAPH 4.5.1 event generator [58]. This model includes spin correlations as well as finite-width effects. For each $W^{\prime}$-boson mass hypothesis, a sample with $g_{W^{\prime}}=g_{W}$ is processed by PYTHIA with the Z2* [59] tune, in order to simulate showering and hadronization. This sample is then reweighted using MADGRAPH in order to simulate the $M_{\mathrm{T}}$ distribution for different values of the $W^{\prime}$-boson couplings. Both generators simulate at LO. The CTEQ6L1 PDF [48] is employed.

To correct for higher-order effects in $W$-boson production, the difference between the next-to-leading order (NLO) and LO event yields are taken into account in the transverse mass distribution. For the $W^{\prime}$-boson contribution, the LO cross sections have been used.

\section{E. Split-UED model}

The leptonic final states under study may also be interpreted in the framework of universal extra dimensions 
with fermions propagating in the bulk, known as split-UED $[9,10]$. This is a model based on an extended space-time with an additional compact fifth dimension of radius $R$. In this model all SM particles have corresponding KaluzaKlein $(\mathrm{KK})$ partners, for instance $W_{\mathrm{KK}}^{(\mathrm{n})}$, where the superscript denotes the $n$th KK excitation mode. Only KK-even modes of $W_{\mathrm{KK}}^{(\mathrm{n})}$ couple to SM fermions, owing to KK-parity conservation. Modes with $n \geq 4$ are not expected to be accessible under present LHC conditions, and hence the only mode considered is $n=2$. Interference with the $\mathrm{SM}$ is not considered in this model. Under this assumption, the decay to leptons is kinematically identical to the SSM $W^{\prime}$-boson decay, and the observed limits obtained from the $W^{\prime} \rightarrow e \nu$ and $W^{\prime} \rightarrow \mu \nu$ searches can be reinterpreted directly in terms of the $W_{\mathrm{KK}}^{(\mathrm{n})}$-boson mass, taking into account the difference in widths in the simulation. The LO and NNLO production cross sections for a $W_{\mathrm{KK}}^{(2)}$-boson are shown in Table II.

The UED model is parametrized by the quantities $R$ and $\mu$, which are the radius of the extra dimension and the bulk mass parameter of the fermion field in five dimensions. In the split-UED model, the parameter $\mu$ is assumed to be nonzero, following Refs. $[9,10]$.

The mass of the $W_{\mathrm{KK}}^{(\mathrm{n})}$ is determined by $M_{W_{\mathrm{KK}}^{(\mathrm{n})}}=$ $\sqrt{M_{W}^{2}+(n / R)^{2}}$; i.e., a larger radius corresponds to smaller KK masses. The mass of KK fermions depends on the bulk mass parameter $\mu$. The cross section of the $W_{\mathrm{KK}}^{(\mathrm{n})}$ production times the branching fraction to standard model fermions goes to zero as $\mu$ goes to zero.

\section{F. Model with a $\mathbf{T e V}^{-1}$ extra dimension}

Another extra dimensions model, the $\mathrm{TeV}^{-1}$ model, has been proposed [11-14], in which only the fermions are confined to ordinary three-dimensional space, with the SM gauge bosons and the Higgs field propagating in compactified extra dimensions. Under the assumption of a single extra dimension, the model is specified by one parameter $R=1 / M_{\mathrm{C}}$, the size of the compactified dimension, with $M_{\mathrm{C}}$ being the corresponding compactification scale. The $W$-bosons propagating in the compactified dimension are equivalent to the $\mathrm{KK}$ states $W_{\mathrm{KK}}^{(\mathrm{n})}$ with masses $M_{n}=$ $\sqrt{M_{0}^{2}+(n / R)^{2}}$, where $M_{0}$ is the mass of the SM $W$ boson. The coupling constant of the KK states (for $n>0$ ) to fermions is $\sqrt{2}$ times larger than that of the SM $W$-boson.

The signal is similar in shape to an SSM $W^{\prime}$-boson with destructive interference, as seen in Fig. 3 (bottom). This results in effective cross sections of $-0.966 \pm 0.025 \mathrm{fb}$ for the $M_{\mathrm{C}}=2.0 \mathrm{TeV}$ case and $-0.079 \pm 0.014 \mathrm{fb}$ for the $M_{\mathrm{C}}=2.6 \mathrm{TeV}$ case with respect to the $W$-boson.

Given the absence of higher-order calculations, the samples are generated at LO with MADGRAPH 5.1.3.22 using the CTEQ6L1 parton distribution functions. The cross sections with the $W$-boson cross section subtracted are summarized in Table II.

\section{OBJECT IDENTIFICATION AND EVENT SELECTION}

The models described in the previous section provide an event signature of a single high- $p_{\mathrm{T}}$ lepton (electron or muon) and one or more particles that cannot be detected directly (neutrino, dark matter particle) and so give rise to experimentally observed $E_{\mathrm{T}}^{\text {miss }}$. This quantity is measured using a particle-flow technique [60-62], an algorithm that combines measurements from all components of the CMS detector in order to produce particle candidates. The modulus of the vector $p_{\mathrm{T}}$ sum of these candidates defines $E_{\mathrm{T}}^{\text {miss }}$, which is corrected for the jet energy calibration $[63,64]$. At high $M_{\mathrm{T}}$, the calculation of $E_{\mathrm{T}}^{\text {miss }}$ is dominated by the high- $p_{\mathrm{T}}$ lepton in the event.

Candidate events with at least one high- $p_{\mathrm{T}}$ lepton are selected using single-muon (with $p_{\mathrm{T}}>40 \mathrm{GeV}$ ) and single-electron (with $E_{\mathrm{T}}>80 \mathrm{GeV}$ ) triggers and loose electron identification criteria. The relatively high electron trigger threshold is required in order to suppress nonprompt electrons and jets. In the muon channel, the offline reconstructed $p_{\mathrm{T}}$ must be greater than $45 \mathrm{GeV}$, where the trigger is already fully efficient. This relatively low $p_{\mathrm{T}}$ requirement does not impair the search in the high- $M_{\mathrm{T}}$ region, while preserving an adequate number of events in the low- and medium- $M_{\mathrm{T}}$ control regions. The requirement of $E_{\mathrm{T}}>100 \mathrm{GeV}$ in the electron channel ensures a constant and high trigger efficiency. The trigger efficiency for single electrons has been determined with "tag-and-probe" methods [65] to be 99.1 (97.6)\% for the barrel (end cap) ECAL, with a data-to-simulation scale factor of nearly 1 . The single-muon trigger efficiency varies from $94 \%$ in the barrel to $82 \%$ in the end cap regions, with data-tosimulation scale factors of 0.98-0.96 [66].

Electrons are reconstructed as ECAL clusters that are matched to a tracker track, and their identification has been optimized for high $p_{\mathrm{T}}$ [28]. They have to be sufficiently isolated, have an electronlike shape, and be within the acceptance region of the barrel $(|\eta|<1.442)$ or the end caps $(1.56<|\eta|<2.5)$. This acceptance region avoids the gap between the barrel and end cap, where the misidentification probability is the highest. Electron isolation in the tracker is ensured by requiring the $p_{\mathrm{T}}$ sum of all tracks that are in close proximity to the track of the electron candidate and originate from the same primary vertex, to be less than $5 \mathrm{GeV}$. Here, only tracks that are within a cone of $\Delta R=0.3$ around the electron candidate's track are considered. The primary vertex is defined as the vertex with the highest $\sum p_{\mathrm{T}}^{2}$ in the event, where the sum extends over the charged tracks associated with the vertex. In the calorimeters, the $E_{\mathrm{T}}$ sum of energy deposits around the electron candidate is 
used as a measure of isolation. It is corrected for the mean energy contribution from additional proton-proton collisions during the same bunch crossing (pileup). As in the tracker isolation calculation, contributions within a $\Delta R<0.3$ cone around the electron candidate are considered. To obtain sufficiently isolated electrons, this calorimeter isolation is required to be below a threshold of around $3 \%$ of the electron's $E_{\mathrm{T}}$. Additionally, the energy deposits in the hadron calorimeter within a cone of $\Delta R=0.15$ around the electron's direction must be less than $5 \%$ of the electron's energy deposit in the ECAL. To differentiate between electrons and photons, properties of the track matched to the calorimeter measurement must be consistent with those of a prompt electron. Specifically, there must be $\leq 1$ hit missing in the innermost tracker layers, and the transverse distance to the primary vertex must be $<0.02 \mathrm{~cm}$ (barrel) or $<0.05 \mathrm{~cm}$ (end cap). To reduce the Drell-Yan background, events with additional electrons of $E_{\mathrm{T}}>35 \mathrm{GeV}$ are rejected.

The reconstruction of muons is optimized for high $p_{\mathrm{T}}$. Information from the inner tracker and the outer muon system are used together. Each muon is required to have at least one hit in the pixel detector, at least six tracker layer hits, and segments in two or more muon detector layers. Since segments are typically found in consecutive layers separated by thick layers of steel, the latter requirement significantly reduces the amount of hadronic punchthrough [29]. To reduce background from cosmic ray muons, each muon is required to have a transverse impact parameter $\left|d_{0}\right|$ of less than $0.02 \mathrm{~cm}$ and to have a longitudinal distance parameter $\left|d_{z}\right|$ of the tracker track of less than $0.5 \mathrm{~cm}$. Both parameters are defined with respect to the primary vertex. To suppress muons with mismeasured $p_{\mathrm{T}}$, an additional requirement $\sigma_{p_{\mathrm{T}}} / p_{\mathrm{T}}<0.3$ is applied, where $\sigma_{p_{\mathrm{T}}}$ is the uncertainty from the track reconstruction. To match the trigger acceptance, the muon must have $|\eta|<2$.1. Muon isolation requires that the scalar $p_{\mathrm{T}}$ sum of all tracks originating from the interaction vertex within a $\Delta R=\sqrt{(\Delta \phi)^{2}+(\Delta \eta)^{2}}<0.3$ cone around its direction, excluding the muon itself, is less than $10 \%$ of the muon's $p_{\mathrm{T}}$. To further reduce the Drell-Yan and cosmic ray backgrounds, the event must not have a second muon with $p_{\mathrm{T}}>25 \mathrm{GeV}$.

The reconstruction efficiencies for both electrons and muons are measured using same-flavor dilepton events, up to the highest accessible $p_{\mathrm{T}}$. Data and simulation agree within statistical uncertainties for these high-energy events. For higher $p_{\mathrm{T}}$, the flat efficiency is extrapolated and assigned an associated systematic uncertainty, as described in Sec. VII.

To identify any differences in selection efficiency for observed and simulated data, efficiencies for both are determined using the tag-and-probe method. The total efficiency in each case includes contributions from the trigger, lepton identification, and isolation criteria. The ratio of data to simulation efficiencies, denoted as the scaling factor (SF), is determined to be $0.975 \pm 0.023$ $(0.970 \pm 0.042)$ for the barrel (end cap) in the electron channel. For the muon channel, the SFs are $0.967 \pm 0.026$ for $|\eta|<0.9$ (barrel), $0.948 \pm 0.026$ for $0.9<|\eta|<1.2$ (barrel-end cap interface), and $0.979 \pm 0.026$ for $1.2<|\eta|<2.1$ (forward region) [66].

In the models considered, the lepton and $\vec{p}_{\mathrm{T}}^{\text {miss }}$ are expected to be nearly back to back in the transverse plane, and balanced in transverse energy. This is illustrated in Fig. 4 for the muon channel for three example signals (SSM, HNC-CI, and DM) with an $M_{\mathrm{T}}$ threshold of $220 \mathrm{GeV}$. To incorporate these characteristics in the analysis, additional kinematic criteria select events based
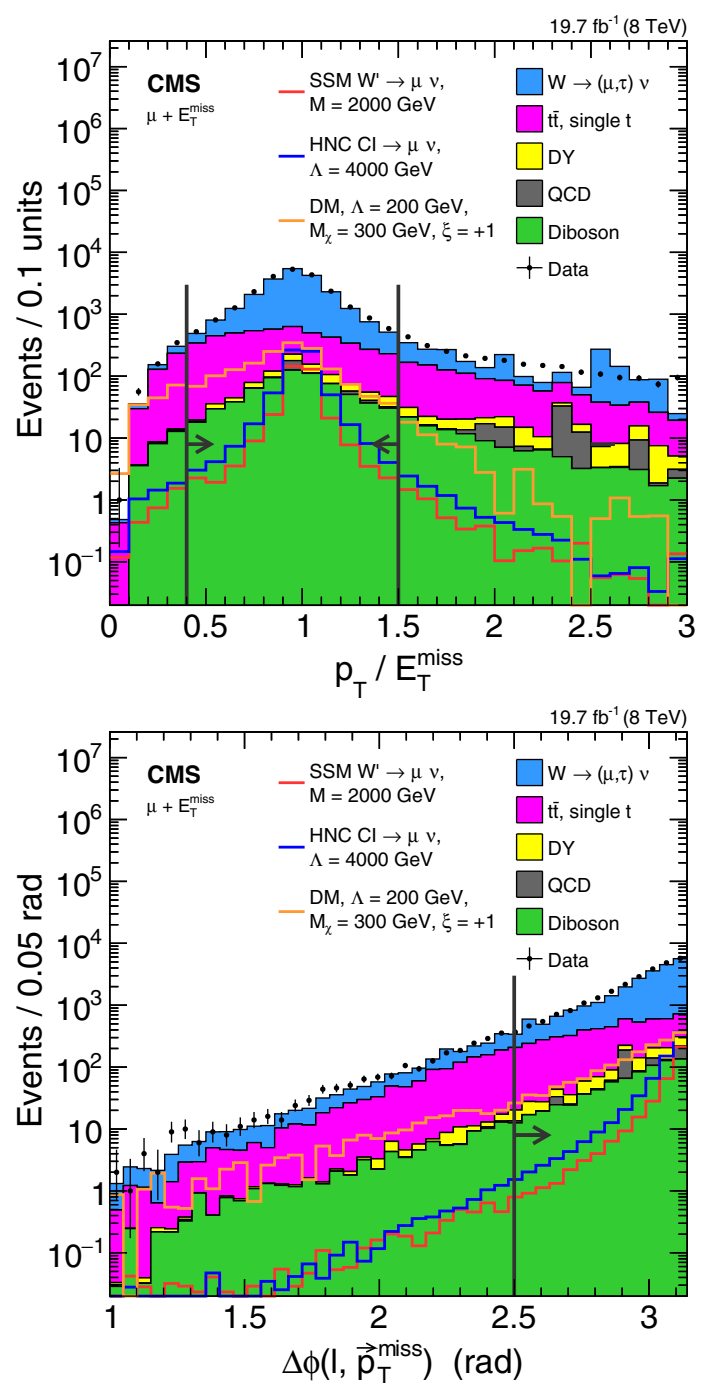

FIG. 4 (color online). The distribution in $p_{\mathrm{T}} / E_{\mathrm{T}}^{\mathrm{miss}}$ (top) and $\Delta \phi\left(\ell, \vec{p}_{\mathrm{T}}^{\text {miss }}\right)$ (bottom), for data, background, and some signals in the muon channel with an $M_{\mathrm{T}}$ threshold of $220 \mathrm{GeV}$. The simulated background labeled as "diboson" includes $W W, Z Z$, and WZ contributions, while "DY" denotes the Drell-Yan process. 
TABLE III. Signal efficiencies for the various models.

\begin{tabular}{lccc}
\hline \hline Model & Parameter & Electron channel & Muon channel \\
\hline SSM $W^{\prime}$ & $\mathrm{M}_{W^{\prime}}=0.5 \mathrm{TeV}$ & $62 \pm 3 \%$ & $64 \pm 3 \%$ \\
SSM $W^{\prime}$ & $\mathrm{M}_{W^{\prime}}=1.5 \mathrm{TeV}$ & $74 \pm 6 \%$ & $71 \pm 7 \%$ \\
SSM $W^{\prime}$ & $\mathrm{M}_{W^{\prime}}=4 \mathrm{TeV}$ & $50 \pm 5 \%$ & $55 \pm 4 \%$ \\
HNC-CI & $\Lambda$ independent & $80 \pm 6 \%$ & $80 \pm 6 \%$ \\
DM & $\xi=-1$ & $42 \pm 4 \%$ & $42 \pm 4 \%$ \\
DM & $\xi=0$ & $39 \pm 4 \%$ & $39 \pm 4 \%$ \\
DM & $\xi=+1$ & $12 \pm 2 \%$ & $13 \pm 2 \%$ \\
\hline \hline
\end{tabular}

on the ratio of the lepton $p_{\mathrm{T}}$ to $E_{\mathrm{T}}^{\text {miss }}$, requiring $0.4<p_{\mathrm{T}} / E_{\mathrm{T}}^{\mathrm{miss}}<1.5$, and on the angular difference between the lepton and $\vec{p}_{\mathrm{T}}^{\text {miss }}$, with $\Delta \phi\left(\ell, \vec{p}_{\mathrm{T}}^{\text {miss }}\right)>$ $2.5 \approx 0.8 \pi$.

Signal efficiencies are model dependent, determined by the signal shape in the distribution of $M_{\mathrm{T}}$. For simulated events passing all the selection criteria, the average signal efficiencies for a given parameter are summarized in Table III.

The SSM $W^{\prime}$ has maximal signal efficiency at the mass of $1.5 \mathrm{TeV}$, decreasing gradually for larger and smaller masses. The uncertainties quoted in Table III are explained in Sec. VII. The geometrical acceptance is roughly $90 \%$ for both electron and muon channels. For the higher $W^{\prime}$-boson masses up to $4 \mathrm{TeV}$, the signal efficiencies slowly decrease to $50 \%$, because of an increasing fraction of off-shell $W^{\prime}$-bosons.

For the HNC-CI model, the signal efficiency is independent of the interaction scale $\Lambda$ and has been determined from simulation to be $80 \%$ with $6 \%$ uncertainty for both the $e+E_{\mathrm{T}}^{\mathrm{miss}}$ signal and the $\mu+E_{\mathrm{T}}^{\mathrm{miss}}$ signals.

For the DM models, the signal efficiency depends on the steepness of the $M_{\mathrm{T}}$ distribution and the total cross section, both of which are sensitive to the interference parameter $\xi$. For $\xi=+1$, the spectrum falls more rapidly, and the search region corresponds to the low-to-medium part of the $M_{\mathrm{T}}$ spectrum, resulting in a rather low signal efficiency of $(13 \pm 2) \%$. For the other two interference cases, $\xi=0$ and -1 , the spectrum extends to very high $M_{\mathrm{T}}$, where the expected background is negligible and the electron and muon channel signal efficiencies are as high as (39 \pm 4$) \%$ for $\xi=0$ and $(42 \pm 4) \%$ for $\xi=-1$. As expected, no difference in efficiency is observed between the vector and the axial-vector couplings.

\section{DISTRIBUTION IN $M_{\mathrm{T}}$ INVARIANT MASS}

The observed $M_{\mathrm{T}}$ distributions for the analyzed data sets are shown in Fig. 5 for the electron and muon channels. Included in the same figure are the predicted $M_{\mathrm{T}}$ distributions for the accepted SM events, separated into contributions from each background process, along with example signal distributions for DM, SSM $W^{\prime}$, and HNC-CI models. For both channels, a variable binning commensurate with
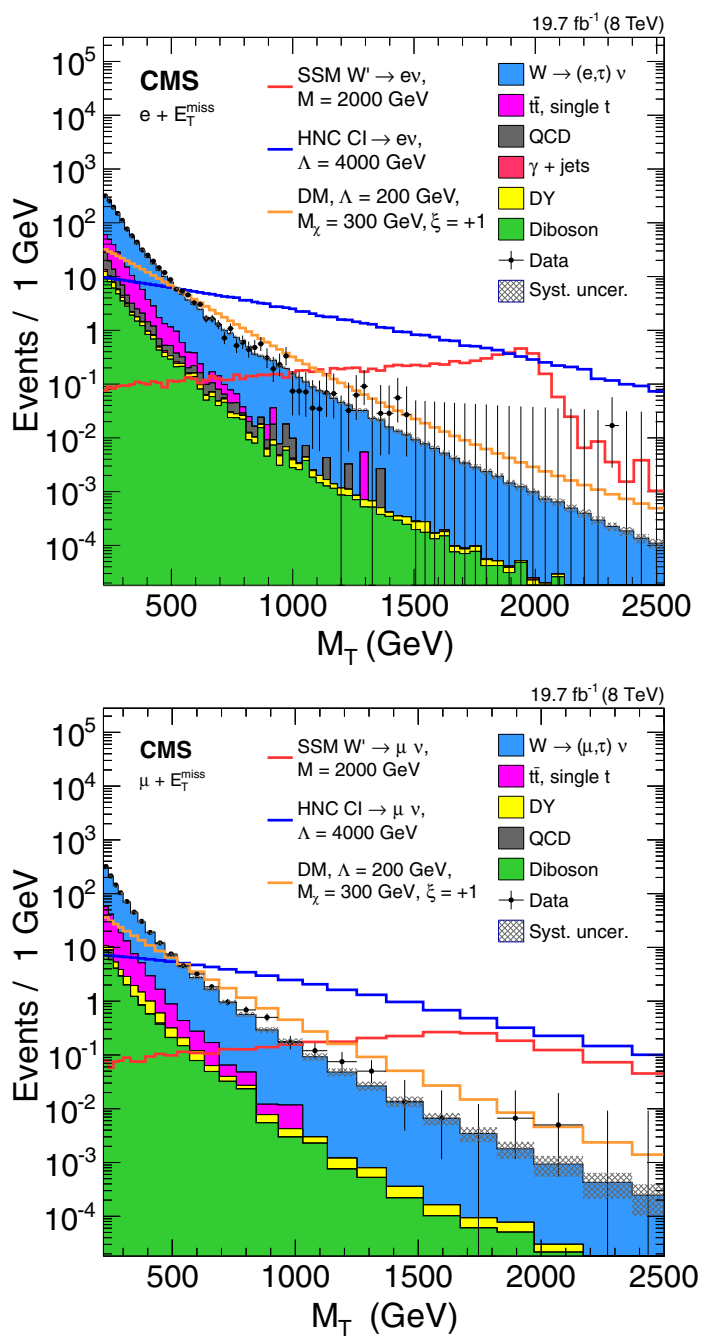

FIG. 5 (color online). Observed $M_{\mathrm{T}}$ distributions for the electron (top) and muon (bottom) channels. The horizontal bars on the data points indicate the widths of the bins. The asymmetric error bars indicate the central confidence intervals for Poissondistributed data and are obtained from the Neyman construction as described in Ref. [67].

the energy-dependent $M_{\mathrm{T}}$ resolution is used. The expected systematic uncertainty in the $M_{\mathrm{T}}$ distribution is also shown.

The deviation of the data from the standard model prediction is shown in Fig. 6. No significant deviation from the predicted background is observed in the $M_{\mathrm{T}}$ spectrum. The highest transverse mass events observed have $M_{\mathrm{T}}=2.3 \mathrm{TeV}$ in the electron channel and $M_{\mathrm{T}}=$ $2.1 \mathrm{TeV}$ in the muon channel. Both events have a wellreconstructed high- $p_{\mathrm{T}}$ lepton and very little hadronic activity.

\section{BACKGROUND}

\section{A. Sources of background}

The primary source of background for all signals is the presence of off-peak, high-transverse-mass tails of the SM 

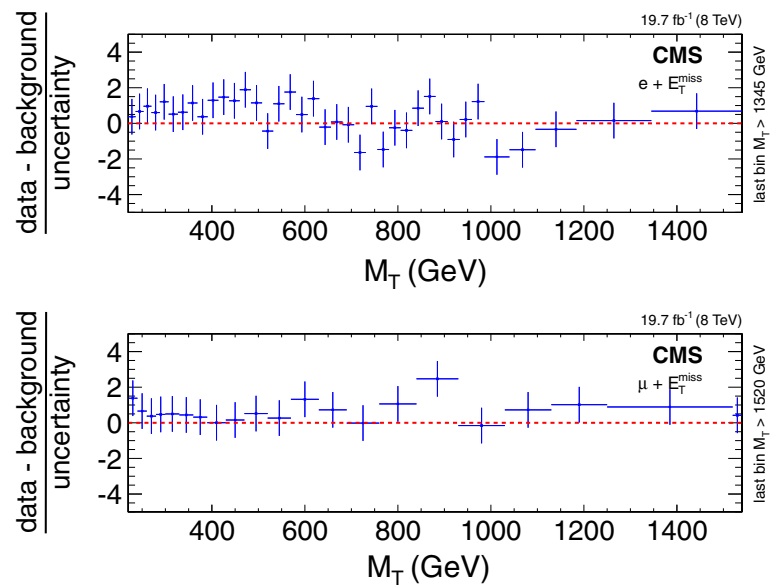

FIG. 6 (color online). Deviation of the number of observed events from the expectation, as shown in Fig. 5, in units of standard deviations, binned in $M_{\mathrm{T}}$ for the electron (top) and muon (bottom) channels. Both the systematic and statistical uncertainties are included. The horizontal bars on the data points indicate the widths of the bins, which are chosen as in Fig. 5 with the extension to include always five background events in a bin.

$W \rightarrow \ell \nu$ decays. Other important backgrounds arise from QCD multijet, $t \bar{t}$, and Drell-Yan events. Contributions from dibosons ( $W W, W Z, Z Z$ ) decaying to $e^{-}, \mu$-, or $\tau$-leptons are also considered. The following background sources are considered in this analysis. They are listed in the order of Fig. 5, where their distribution in $M_{\mathrm{T}}$ can be seen.

(1) A $W \rightarrow \ell \nu$ with $\ell=e, \mu$ sample is simulated at $\mathrm{LO}$ with PYTHIA. To ensure a good description of the considered phase space, two samples with lepton $p_{\mathrm{T}}$ ranges $100-500 \mathrm{GeV}$ and $\geq 500 \mathrm{GeV}$, respectively, are used. A transverse-mass-dependent $\mathrm{K}$-factor is calculated, including NLO QCD and electroweak corrections (see Sec. VIC).

(2) A $W \rightarrow \tau \nu$ sample, where the $\tau$-lepton decays to an electron or a muon and the two corresponding neutrinos, is generated with PYTHIA using the same $\mathrm{K}$-factors as above. Electrons and muons from these decays have a small impact parameter with respect to the primary vertex and are not separable from prompt leptons. In addition, the $p_{\mathrm{T}} / E_{\mathrm{T}}^{\text {miss }}$ ratio is approximately 1 , despite the presence of three neutrinos in the event, since the vector sum of the neutrinos' $p_{\mathrm{T}}$ balances the charged lepton $p_{\mathrm{T}}$ for small $W$-boson $p_{\mathrm{T}}$. These features prevent an efficient rejection of $W \rightarrow \tau \nu$ events, which therefore contribute to the background, albeit at low $M_{\mathrm{T}}$ (see Fig. 7) and with little contribution in the high- $M_{\mathrm{T}}$ region.

(3) Top-quark pair and single top-quark production are other sources of high- $p_{\mathrm{T}}$ leptons and $E_{\mathrm{T}}^{\text {miss }}$, and these are generated with MC@NLO $[68,69]$ in combination with HERWIG [70], and POWHEG [71-74] in combination with PYTHIA, respectively. A newly calculated

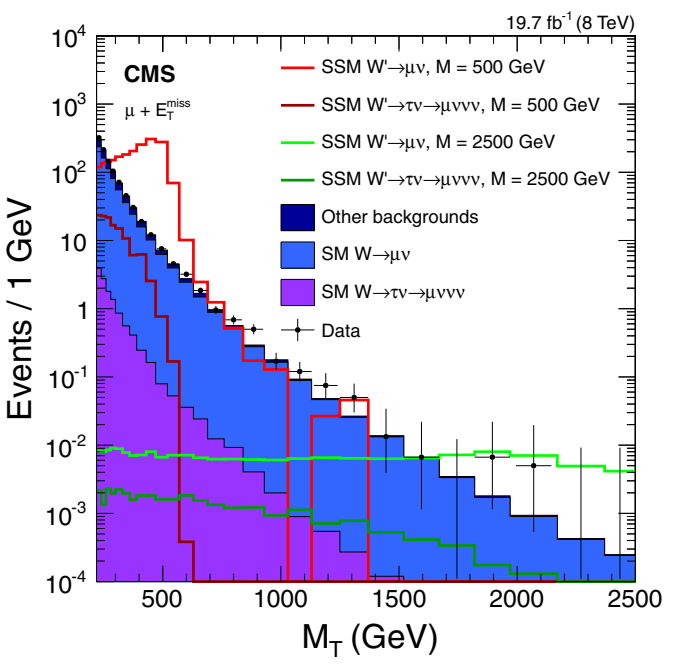

FIG. 7 (color online). Contributions from $W$ - and $W^{\prime}$-bosons decaying to $\tau \nu \rightarrow \mu \nu \nu \nu$ compared to the prompt $\mu \nu$ decay channel. Also shown are the contributions from the other backgrounds and the number of data events. The horizontal bars on the data points indicate the widths of the bins.

NNLO cross section [75] is used to rescale the NLO predictions. These events are largely rejected by the requirement of two-body decay kinematics (see Sec. IV) but can extend into high $M_{\mathrm{T}}$ as seen in Fig. 5.

(4) Multijet background (QCD), enriched in electrons/ photons and muons, is generated with PYTHIA. Although this process has by far the largest cross section, it is efficiently rejected by the isolation requirements imposed to select the lepton candidates as well as the requirement on the ratio of $p_{\mathrm{T}} / E_{\mathrm{T}}^{\text {miss }}$ (see Sec. IV). Despite the large suppression of these events, the misidentification of jets as leptons (especially as electrons) still occurs. The contribution of QCD multijet events to the electron channel is derived from data as explained in Sec. VID.

(5) Drell-Yan production of dileptons $(\ell=e, \mu)$ constitutes a background when one lepton escapes detection. The samples are generated with POWHEG [76]. Contributions from Drell-Yan production of $\tau \bar{\tau}$ are simulated using PYTHIA, applying a uniform QCD K-factor of 1.26 (calculated with FEWZ [49,50]).

(6) Contributions from dibosons ( $W W, W Z, Z Z)$ decaying to a state with at least one lepton are generated with PYTHIA and scaled to NLO cross sections.

(7) In the electron channel, a $\gamma+$ jet event sample, generated with PYTHIA, is used to estimate the effects of photons misidentified as electrons.

Background selection efficiencies for $M_{\mathrm{T}}>220 \mathrm{GeV}$ are summarized in Table IV. The total background predictions, listed in Table V, are comparable in the electron and muon channels. Resolution effects, reconstruction 
TABLE IV. Background processes and their number of events $N_{\text {events }}$ and selection efficiencies after the full selection and the requirement $M_{\mathrm{T}}>220 \mathrm{GeV}$.

\begin{tabular}{lcccc}
\hline \hline Process & \multicolumn{2}{c}{$e$ channel } & \multicolumn{2}{c}{$\mu$ channel } \\
& $N_{\text {events }}$ & Selection efficiency & $N_{\text {events }}$ & Selection efficiency \\
\hline$W \rightarrow \ell \nu$ with $\ell=e, \mu$ & $18390 \pm 170$ & $(745.8 \pm 7.0) \times 10^{-7}$ & $17261 \pm 151$ & $(697.5 \pm 6.1) \times 10^{-7}$ \\
$W \rightarrow \tau \nu, \tau \rightarrow(e, \mu) \nu \nu$ & $491 \pm 74$ & $(19.8 \pm 3.0) \times 10^{-7}$ & $281.3 \pm 3.4$ & $(113.7 \pm 1.7) \times 10^{-8}$ \\
Top-quark pair and Single top-quark & $2831 \pm 28$ & $(398.4 \pm 3.9) \times 10^{-6}$ & $3132 \pm 30$ & $(486.8 \pm 4.6) \times 10^{-6}$ \\
Diboson & $783.8 \pm 8.3$ & $(376.5 \pm 4.0) \times 10^{-6}$ & $629.6 \pm 7.7$ & $(325.8 \pm 4.0) \times 10^{-6}$ \\
Multijet $(\mathrm{QCD})$ & $705 \pm 19$ & Data driven & $16.5 \pm 7.3$ & $(11.1 \pm 4.9) \times 10^{-12}$ \\
$\mathrm{DY} \rightarrow \ell \ell$ with $\ell=e, \mu, \tau$ & $159 \pm 19$ & $(20.8 \pm 2.5) \times 10^{-7}$ & $216.1 \pm 5.9$ & $(280.8 \pm 7.6) \times 10^{-8}$ \\
$\gamma+$ jet & $56.7 \pm 8.4$ & $(31.7 \pm 4.7) \times 10^{-7}$ & - & - \\
\hline \hline
\end{tabular}

efficiencies, and statistical fluctuations are taken into account. Systematic uncertainties are discussed in Sec. VII.

All simulated event samples are normalized to the integrated luminosity of the recorded data, using calculated NNLO cross sections. The only exceptions are the diboson and QCD samples, for which the NLO and LO cross sections are used, respectively. The simulation of pileup is included in all event samples by superimposing minimum bias interactions onto all simulated events. For the data set used, the average number of interactions per bunch crossing is 21 .

\section{B. Prediction of the expected background}

Searching for deviations from the steeply falling $W$-boson $M_{\mathrm{T}}$ spectrum requires an accurate background estimate at very high transverse masses. Several methods are used to evaluate the expected background in the signal region, based either on control samples in data or on a fully simulation-based approach.

For the majority of background sources, the estimate is determined from simulation, based on samples with large event counts at high $M_{\mathrm{T}}$. However, to avoid bin-to-bin statistical fluctuations that may still occur at very high $M_{\mathrm{T}}$, the total background prediction is parametrized with the empirical function given in Eq. (3),

$$
f\left(M_{\mathrm{T}}\right)=e^{a+b M_{\mathrm{T}}+c M_{\mathrm{T}}^{2}} M_{\mathrm{T}}^{d},
$$

where $a, b, c$, and $d$ are the fit parameters. This function was found to provide a good description of the steeply falling SM background up to high $M_{\mathrm{T}}$. Based on this

TABLE V. Event yields observed in data, and expected from background and signal, for different transverse mass thresholds. The quoted uncertainties are the combined uncertainties assuming a log-normal distribution, not including a $2.6 \%$ integrated luminosity uncertainty [25].

\begin{tabular}{|c|c|c|c|c|}
\hline & & $M_{\mathrm{T}}>1.0 \mathrm{TeV}$ & $M_{\mathrm{T}}>1.5 \mathrm{TeV}$ & $M_{\mathrm{T}}>2.0 \mathrm{TeV}$ \\
\hline \multicolumn{5}{|c|}{ Electron channel } \\
\hline Data & & 24 & 1 & 1 \\
\hline SM Background & & $26.0_{-25}^{+2.5}$ & $2.02_{-0.25}^{+0.26}$ & $0.207_{-0.033}^{+0.036}$ \\
\hline \multirow[t]{2}{*}{$W^{\prime}$} & $M_{W^{\prime}}=2.5 \mathrm{TeV}$ & $50.5_{-7.5}^{+7.5}$ & $38.8_{-6.1}^{+6.23}$ & $24.0_{-3.9}^{+3.9}$ \\
\hline & $M_{W^{\prime}}=3 \mathrm{TeV}$ & $10.3_{-2.1}^{+2.1}$ & $7.8_{-1.9}^{+1.9}$ & $5.8_{-1.5}^{+1.5}$ \\
\hline \multirow[t]{2}{*}{ HNC-CI } & $\Lambda=4 \mathrm{TeV}$ & $1120_{-110}^{+110}$ & $368_{-47}^{+47}$ & $105_{-19}^{+19}$ \\
\hline & $\Lambda=9 \mathrm{TeV}$ & $43.4_{-4.3}^{+4.3}$ & $14.3_{-1.8}^{+1.8}$ & $4.08_{-0.75}^{+0.75}$ \\
\hline \multirow[t]{3}{*}{ DM vector coupling, $M_{\chi}=50 \mathrm{GeV}, \Lambda=300$} & $\xi=+1$ & $0.402_{-0.050}^{+0.050}$ & $0.0346_{-0.0070}^{+0.0072}$ & $0.0033_{-0.0010}^{+0.0010}$ \\
\hline & $\xi=0$ & $6.8_{-1.5}^{+1.5}$ & $1.25_{-0.42}^{+0.42}$ & $0.22_{-0.11}^{+0.11}$ \\
\hline & $\xi=-1$ & $27.4_{-5.9}^{+5.9}$ & $5.0_{-1.7}^{+1.7}$ & $0.89_{-0.43}^{+0.44}$ \\
\hline \multicolumn{5}{|c|}{ Muon channel } \\
\hline Data & & 35 & 3 & 1 \\
\hline SM background & & $26.1_{-4.3}^{+4.4}$ & $2.35_{-0.60}^{+0.70}$ & $0.33_{-0.12}^{+0.16}$ \\
\hline \multirow[t]{2}{*}{$W^{\prime}$} & $M_{W^{\prime}}=2.5 \mathrm{TeV}$ & $48.7_{-4.1}^{+4.1}$ & $36.1_{-3.1}^{+2.8}$ & $20.3_{-3.4}^{+3.0}$ \\
\hline & $M_{W^{\prime}}=3 \mathrm{TeV}$ & $9.88_{-0.98}^{+0.99}$ & $7.33_{-0.65}^{+0.64}$ & $5.00_{-0.39}^{+0.16}$ \\
\hline \multirow[t]{2}{*}{ HNC-CI } & $\Lambda=9 \mathrm{TeV}$ & $42.4_{-3.8}^{+3.8}$ & $13.8_{-2.0}^{+0.03}$ & $4.47_{-0.94}^{+0.99}$ \\
\hline & $\Lambda=4 \mathrm{TeV}$ & $1091_{-98}^{+97}$ & $356_{-52}^{+50}$ & $115_{-24}^{+23}$ \\
\hline \multirow[t]{3}{*}{ DM vector coupling, $M_{\chi}=50 \mathrm{GeV}, \Lambda=300$} & $\xi=+1$ & $0.271_{-0.067}^{+0.070}$ & $0.0151_{-0.0056}^{+0.0061}$ & $0.00088_{-0.00043}^{+0.00051}$ \\
\hline & $\xi=0$ & $6.7_{-1.6}^{+1.6}$ & $1.43_{-0.51}^{+0.54}$ & $0.31_{-0.15}^{+0.17}$ \\
\hline & $\xi=-1$ & $27.1_{-6.5}^{+6.6}$ & $5.8_{-2.1}^{+2.2}$ & $1.25_{-0.60}^{+0.68}$ \\
\hline
\end{tabular}


parametrization, the expected number of SM background events for all transverse mass bins can be predicted, as shown for three typical thresholds $M_{\mathrm{T}}^{\text {min }}$ in Table $\mathrm{V}$. Contributions from SM processes drop quickly with increasing $M_{\mathrm{T}}$, leading to an expected yield of less than 0.5 events in each channel, for $M_{\mathrm{T}}>2 \mathrm{TeV}$.

\section{Higher-order corrections for SM $W$-boson background}

The $W$-boson, particularly through the off-shell tail of its $M_{\mathrm{T}}$ distribution, contributes an important and irreducible background in this analysis. An accurate prediction of the $W$-boson's off-shell tail is also needed to establish the effects of interference with the signal. Therefore, higherorder electroweak (EW) and QCD corrections are evaluated, binned in $M_{\mathrm{T}}$ :

$$
K\left(M_{\mathrm{T}}\right)=\frac{\Delta \sigma(\mathrm{NLO}) / \Delta M_{\mathrm{T}}}{\Delta \sigma(\mathrm{LO}) / \Delta M_{\mathrm{T}}}
$$

The NLO EW corrections, calculated with the HORACE [77] event generator using the CT10 [78] PDF set, depend strongly on $M_{\mathrm{T}}$. While the corresponding $\mathrm{K}$-factor is around 1.0 for transverse masses of $300 \mathrm{GeV}$, it decreases to around 0.5 for $M_{\mathrm{T}}=2.5 \mathrm{TeV}$. The QCD corrections are calculated with MC@NLO, also using the CT10 PDF set, and are less $M_{\mathrm{T}}$ dependent, leading to a $\mathrm{K}$-factor ranging from around 1.4 to 1.2. The impact of both these corrections is illustrated in Fig. 8. Also shown is the influence of the PDF set using the LO generator PYTHIA comparing CT10 and CTEQ6L1 [48].

To combine the EW and QCD differential cross sections, two different approaches have been used [79]: an additive and a multiplicative combination. As both the EW and QCD K-factor include the NLO PDF information, a correction must be made to not account for the NLO PDF twice. The combined K-factors are calculated by

$$
\begin{aligned}
& K_{\mathrm{QCD} \oplus \mathrm{EW}}^{\mathrm{CT} 10}=\frac{\left[\frac{\Delta \sigma}{\Delta M_{\mathrm{T}}}\right]_{\mathrm{QCD}}^{\mathrm{CT} 10}+\left[\frac{\Delta \sigma}{\Delta M_{\mathrm{T}}}\right]_{\mathrm{EW}}^{\mathrm{CT} 10}-\left[\frac{\Delta \sigma}{\Delta M_{\mathrm{T}}}\right]_{\mathrm{LO}}^{\mathrm{CT} 10}}{\left[\frac{\Delta \sigma}{\Delta M_{\mathrm{T}}}\right]_{\mathrm{LO}}^{\mathrm{CTEQ}}}, \\
& K_{\mathrm{QCD} 1}^{\mathrm{CT} 10} \otimes \frac{\left[\frac{\Delta \sigma}{\Delta M_{\mathrm{T}}}\right]_{\mathrm{Q}}^{\mathrm{CT} 10}}{\left[\frac{\Delta \sigma}{\Delta M_{\mathrm{T}}}\right]_{\mathrm{LO}}^{\mathrm{CTE}}} \times \frac{\left[\frac{\Delta \sigma}{\Delta M_{\mathrm{T}}}\right]_{\mathrm{EW}}^{\mathrm{CT} 10}}{\left[\frac{\Delta \sigma}{\Delta M_{\mathrm{T}}}\right]_{\mathrm{LO}}^{\mathrm{CTEQ} 10}} \times \frac{\left[\frac{\Delta \sigma}{\Delta M_{\mathrm{T}}}\right]_{\mathrm{LO}}^{\mathrm{CTEQ} 6 \mathrm{~L} 1}}{\left[\frac{\Delta \sigma}{\Delta M_{\mathrm{T}}}\right]_{\mathrm{LO}}^{\mathrm{CT} 10}},
\end{aligned}
$$
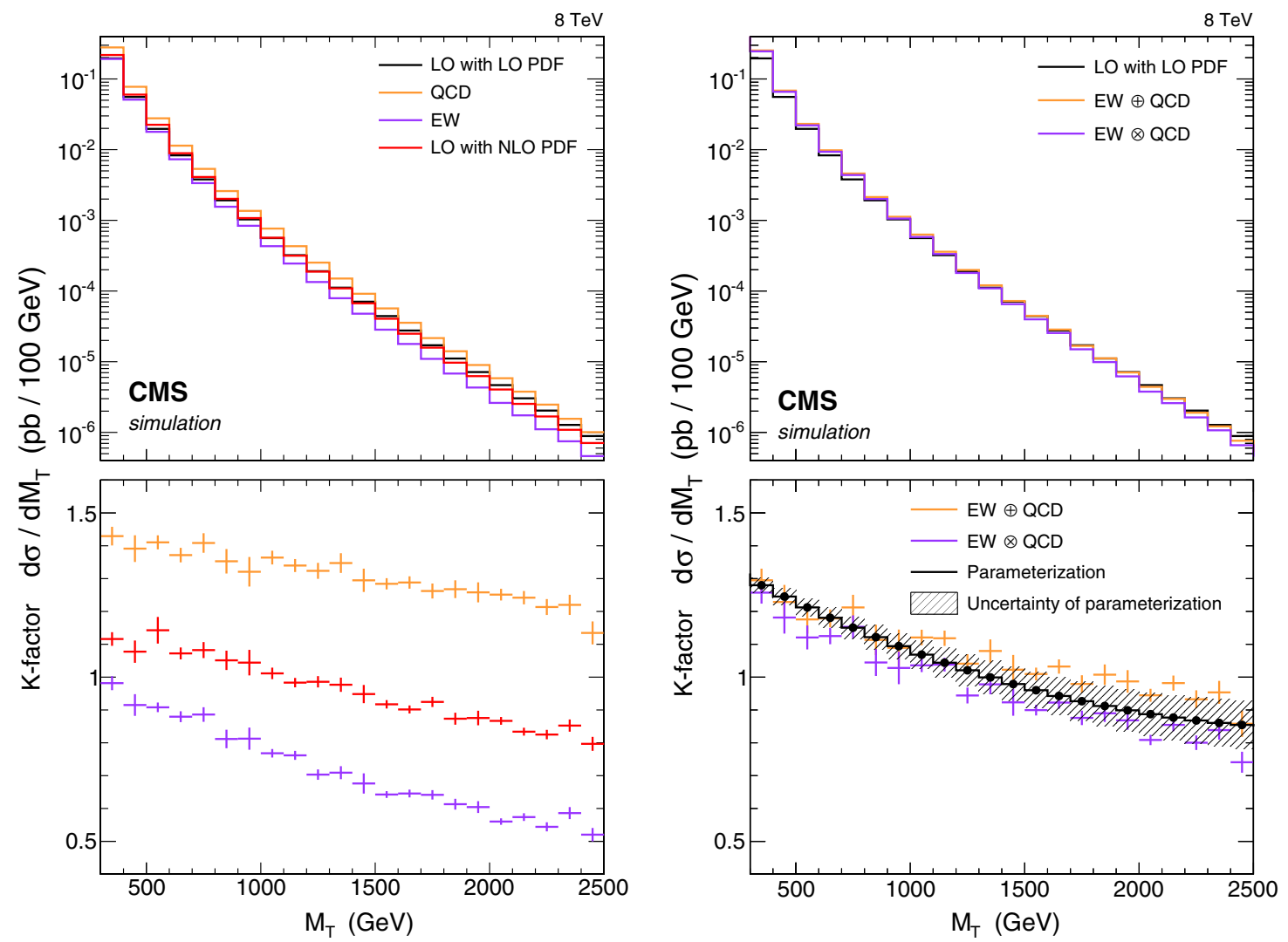

FIG. 8 (color online). Electroweak and QCD corrections to the SM W-boson background prediction. Left: QCD and electroweak contributions compared to the LO calculation with LO PDF. Right: Combination of higher-order corrections with an additive and a multiplicative approach compared to the LO calculation with the LO PDF, as well as a parametrization of the mean of the values obtained by the two methods. To derive the K-factor data on the right plot, one divides the sum or product of the K-factors on the left plot by the NLO/LO PDF K-factor. 
where $\left[\frac{\Delta \sigma}{\Delta M_{\mathrm{T}}}\right]$ is the differential cross section, the upper index gives the PDF set name, and the lower index gives the type of correction applied.

Their effects differ by around $10 \%$. The K-factor assumed in this analysis is obtained by taking the average of the two approaches and treating half of their difference as a systematic uncertainty.

The means of the K-factors resulting from these two approaches are shown in Fig. 8 (right), with the distribution parametrized using a second-order polynomial. These higher-order corrections have a significant influence on the final result. This treatment represents an improvement over previous analyses [21-23], in which a constant $\mathrm{K}$-factor of 1.3 was used across the whole $M_{\mathrm{T}}$ spectrum. For $M_{\mathrm{T}} \geq 1.5 \mathrm{TeV}$ the value used in this analysis is closer to $\sim 0.9$, as shown in the figure. This reduces the expected background, while leaving the signal unchanged since corresponding EW corrections have not been calculated.

\section{Multijet background estimation from data}

As stated in Sec. VIA, the misidentification of jets as leptons, which is more likely for electrons than for muons, is a possible source of background for this search. While the contribution of QCD multijet events to the muon channel is negligible, a small contribution to the electron channel remains. In the latter channel, the shape and normalization of the QCD multijet background, as shown in Fig. 5 and used for the final results, is derived from data.

A QCD template is obtained from the events in which the electron candidate fails the isolation requirement but where all other event requirements are met. QCD template events are scaled with normalization factors from an independent control region, which is defined by the requirement $1.5<E_{\mathrm{T}} / E_{\mathrm{T}}^{\text {miss }}<10$. In this region, the ratio $r_{t t l}$ of "tight" events (electron candidate passes all requirements of a wellisolated electron) to "loose" events (all events in the region) is measured as a function of $E_{\mathrm{T}}$ and $\eta$. The resulting normalization factor for QCD template events is $r_{t t l} /\left(1-r_{t t l}\right)$. Contributions from processes with genuine electrons or photons are estimated via simulation and are subtracted. They amount to $4 \%-13 \%$ of the loose event counts, the most important contributions being $W+$ jets and $\gamma+$ jets events, along with small contributions from $t \bar{t}$, the single top quark, Drell-Yan, and dibosons. This results in ratios of tight to loose event counts varying from $7 \%$ in the barrel to $25 \%$ in the very forward region, for electrons with $E_{\mathrm{T}}>200 \mathrm{GeV}$. Based on a set of cross-checks, a total uncertainty of $40 \%$ is assigned to the multijet background.

\section{SYSTEMATIC UNCERTAINTIES}

Mismeasurement of lepton energy or momentum, resulting from both detector resolution and imperfect scale calibration, will result in a smearing of the $M_{\mathrm{T}}$ spectrum. For each source of uncertainty, shifts of $\pm 1 \sigma$ are applied, the kinematics of the objects $\left(e, \mu, E_{\mathrm{T}}^{\mathrm{miss}}\right)$ are recalculated, as well as $M_{\mathrm{T}}$, and the kinematic selection is reapplied. The resulting distribution is parametrized, and the difference with respect to the original parametrization is used to estimate the systematic uncertainty in the number of background events. The total uncertainty of the expected background is indicated in Fig. 5 and specified in Table V.

The systematic uncertainty in the electron energy scale is estimated to be $0.4(0.8) \%$ in the barrel (end caps). For the electron energy resolution uncertainty, an additional Gaussian smearing of 1.2 (2.4)\% for the barrel (end cap) region is applied to the Monte Carlo (MC) simulation [27].

The muon transverse momentum scale uncertainty is estimated as $5 \% \times p_{\mathrm{T}} / \mathrm{TeV}$. The momentum resolution uncertainty is taken into account by applying an additional smearing of $3.2 \%$ to the MC simulation. Both scale and resolution uncertainty were estimated from the measurement of cosmic muons [29]. The uncertainty of the muon momentum measurement relates to the smallness of curvature of tracks for high- $p_{\mathrm{T}}$ muons, while the energy of the electrons is measured in the crystal calorimeter, and the uncertainty is smaller.

As explained in Sec. VIC, the difference between the two ways of combining the EW and QCD corrections is treated as the systematic uncertainty in the K-factor for $W$-boson production. The effect of even higher-order corrections like Sudakov corrections is expected to be small and therefore not considered.

The ratio of data to MC efficiencies are the SFs defined in Sec. IV. The uncertainty due to the determination method and the extrapolation to high $M_{\mathrm{T}}$ is taken as the systematic uncertainty in the SFs.

The simulated distribution of $p p$ collision vertices per bunch crossing has to be reweighted to the distribution measured in data. The uncertainty due to this reweighting method is treated as the systematic uncertainty of the pileup simulation. The effect on the background event yield due to this uncertainty is smaller than $1 \%$.

The overall uncertainty in the determination of $E_{\mathrm{T}}^{\text {miss }}$ in each event is derived from the individual uncertainties assigned to the objects (jets, $e, \mu, \tau, \gamma$, and unclustered energy) used by the particle-flow algorithm. The contribution of each object type is varied according to its uncertainty. This uncertainty is propagated to the particle-flow $E_{\mathrm{T}}^{\text {miss }}$ [64]. The quadratic sum of the individual uncertainties gives the overall uncertainty in the particleflow $E_{\mathrm{T}}^{\text {miss }}$. To account for correlations, the $E_{\mathrm{T}}^{\text {miss }}$ uncertainty components that are also used for the lepton (e.g., electron energy scale and resolution in the electron channel) are not included in the $E_{\mathrm{T}}^{\text {miss }}$ distributions of Fig. 9 but are included in their respective distributions.

The theoretical uncertainty related to the choice of the PDF set was estimated using the PDF4LHC recommendation $[80,81]$, reweighting the background samples with three different PDF sets: NNPDF2.3 [82], MWST2008 

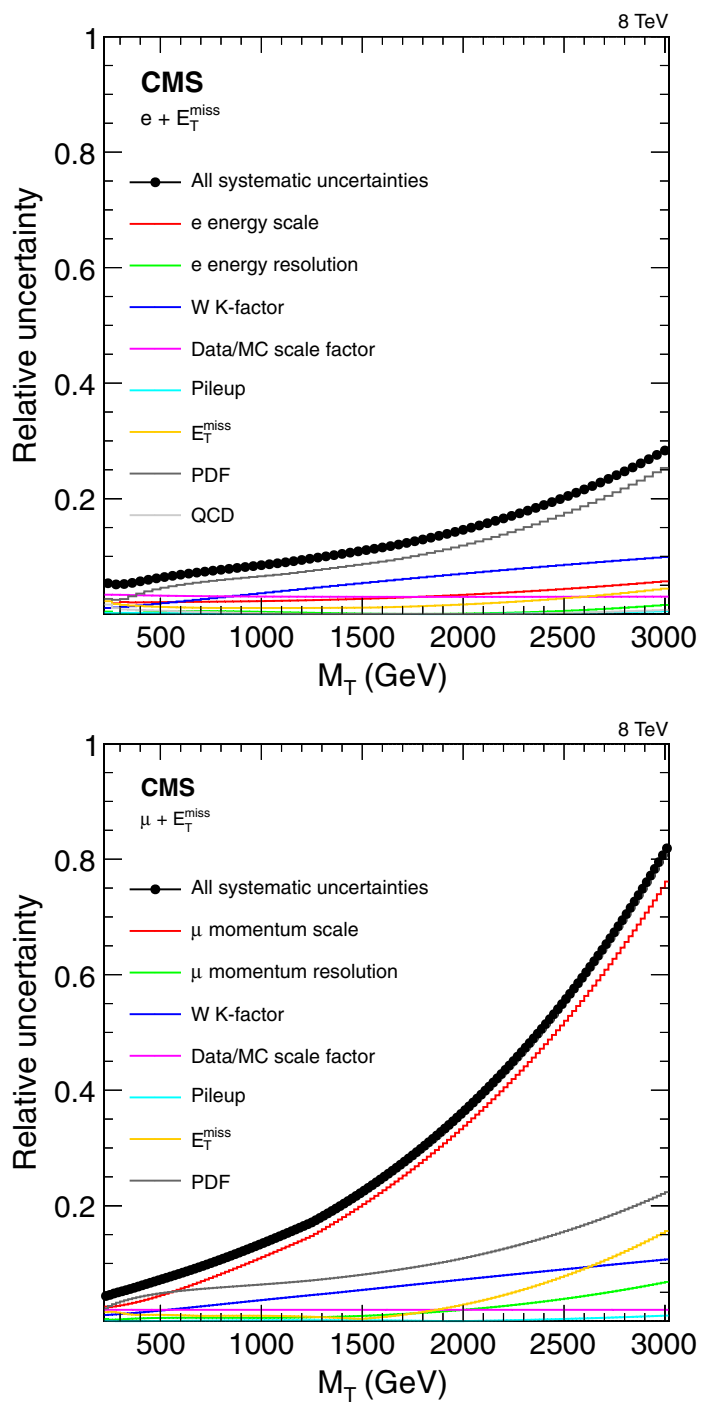

FIG. 9 (color online). Individual contributions of relative systematic uncertainties on the background event yields in the electron (top) and muon (bottom) channels.

[83], and CT10 [78]. For each central PDF set, an uncertainty band is derived from the different error PDF sets. The error PDF sets describe the uncertainties of the PDF set including uncertainties due to $\alpha_{S}$ variation. The envelope of these three error bands is then taken as the uncertainty due to the choice of PDF set. The same procedure was used for the signal cross section predictions.

For the multijet background prediction, the uncertainties described in Sec. VID are used.

The accuracy of the integrated luminosity estimate is $2.6 \%[25]$.

An estimate of the uncertainty in the number of background events in the $M_{T}$ spectrum arising from the uncertainties described above, but not including the luminosity uncertainty, is shown in Fig. 9. The dominant systematic uncertainties are due to the PDF sets in the electron channel and due to momentum scale uncertainty in the muon channel.

\section{LIMIT-SETTING PROCEDURES}

As no significant deviation from predictions is seen in the $M_{\mathrm{T}}$ distribution, exclusion limits on new signals can be set. Upper limits on the production cross section times branching fraction $\sigma_{W^{\prime}} \mathcal{B}\left(W^{\prime} \rightarrow \ell \nu\right)$, with $\ell=e$ or $\mu$, are determined using a Bayesian method [56] with a uniform positive prior probability distribution for the signal cross section. Systematic uncertainties on the expected signal and background yields are included via nuisance parameters with log-normal prior distributions.

To determine a model-independent upper limit on the cross section times branching fraction, all events above a threshold $M_{\mathrm{T}}^{\mathrm{min}}$ are summed. From the number of background events, signal events, and observed data events, the cross section limit can be calculated. No assumptions on the shape of the signal $M_{\mathrm{T}}$ distribution have to be made. This method has a good sensitivity, comparable to a multibin approach, when the background is low.

For the limits on the SSM $W^{\prime}$, HNC-CI, DM, split-UED, and $\mathrm{TeV}^{-1}$ models, the entire $M_{\mathrm{T}}$ spectrum as displayed in Fig. 5 with $M_{\mathrm{T}}>220 \mathrm{GeV}$ selection is considered, taking the shape of the distribution into account using a binned likelihood (multibin counting). This is performed for different values of the model parameters of each signal, resulting in limits in terms of these model parameters, such as the $W^{\prime}$-boson mass or the interaction scale $\Lambda$.

The analyses of the SSMS and SSMO hypotheses are technically challenging, as the number of events in an $M_{\mathrm{T}}$ region can be larger or smaller than predicted by the standard model. For the SSMS model, the $W$ - $W^{\prime}$-boson production cross section would be reduced with respect to the standard model $W$-boson, as seen in Table II, affecting the $M_{\mathrm{T}}$ range below the Jacobian peak as shown in Fig. 3 (top left). An assumption of the overall cross section always influences the whole signal distribution. However, the effect of the SSMS and SSMO signal compared to the SM expectation is to reduce the observed event count in one region, while increasing it in another. This makes the analysis less sensitive in setting a cross section limit. The $W^{\prime}$-boson coupling strength $g_{W^{\prime}}$ has therefore been chosen as a free parameter for the models including $W-W^{\prime}$ interference (SSMS and SSMO). A smaller coupling will result in a narrower Jacobian peak, as well as less modulation in the interference region.

The limits on the $W^{\prime}$-boson coupling strength in the SSMS and SSMO scenarios have been determined using the modified-frequentist $\mathrm{CL}_{\mathrm{s}}$ method $[84,85]$. The test statistic used is

$$
q_{\mu}=-2 \ln \frac{\mathcal{L}\left(\operatorname{data} \mid \mu, \hat{\theta}_{\mu}\right)}{\mathcal{L}\left(\operatorname{data} \mid 0, \hat{\theta}_{0}\right)}
$$


where $\mathcal{L}$ is the likelihood, $\mu$ is the parameter of interest (here, the coupling), and $\hat{\theta}$ is the set of nuisance parameters maximizing the likelihood.

\section{INTERPRETATION OF THE RESULTS}

This section discusses the limits established for the various models summarized in Table I. All limits presented here are at $95 \%$ C.L. unless stated otherwise. Note that all presented mass limits come with a lower bound of $M_{\mathrm{T}}=220 \mathrm{GeV}$, below which background dominates the $M_{\mathrm{T}}$ distribution.
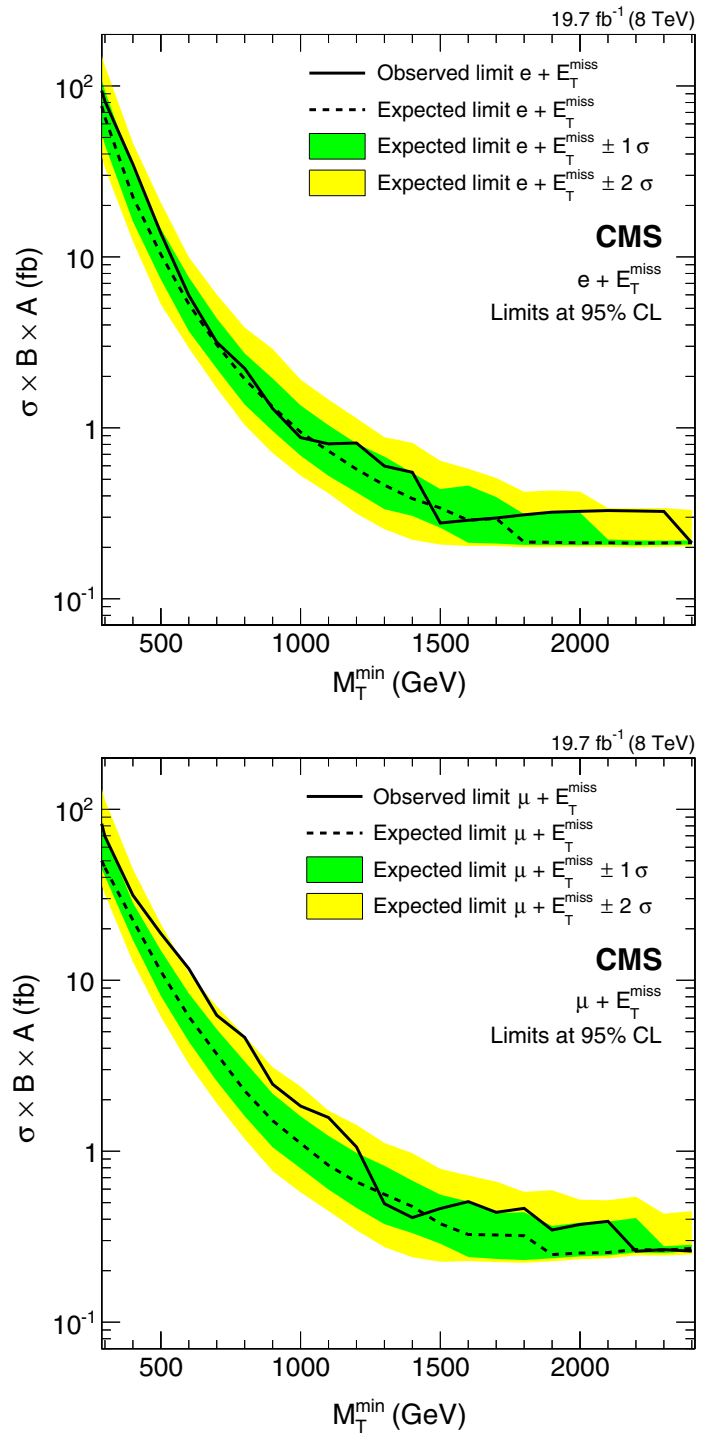

FIG. 10 (color online). Cross section upper limits at $95 \%$ on the effective cross section $\sigma\left(W^{\prime}\right) \mathcal{B}\left(W^{\prime} \rightarrow \ell \nu\right) A$ above a threshold $M_{\mathrm{T}}^{\mathrm{min}}$ for the individual electron and muon channels. Shown are the observed limit, expected limit, and the expected limit $1 \sigma$ and $2 \sigma$ intervals. Only detector acceptance is taken into account for the signal. The parameter $A$ describes the efficiency derived from the kinematic selection criteria and the $M_{\mathrm{T}}^{\min }$ threshold.

\section{A. Model-independent cross section limit}

Apart from the model-dependent multibin limits, a model-independent cross section limit is determined using a single bin ranging from a lower threshold on $M_{\mathrm{T}}$ to infinity, with the results shown in Fig. 10 for the individual electron and muon channels and Fig. 11 for the combination of both channels. Only model-independent contributions to signal efficiencies, e.g., the lepton reconstruction efficiency including detector acceptance, are considered, derived using the simulated $W-\rightarrow \ell \nu$ sample. The signal efficiencies are estimated to be $86 \%$ in the muon channel and $83 \%$ in the electron channel.

To determine any limit for a specific model from the model-independent limit shown here, only the modeldependent part of the efficiency must be taken into account. This means an efficiency $A$ describing the effect on the signal of the $M_{\mathrm{T}}^{\min }$ threshold and of the two kinematic selection criteria, $0.4<p_{\mathrm{T}} / E_{\mathrm{T}}^{\text {miss }}<1.5$ and $\Delta \phi\left(\ell, \vec{p}_{\mathrm{T}}^{\text {miss }}\right)>$ 2.5 (see Sec. IV), must be determined. The $p_{\mathrm{T}}$ and $E_{\mathrm{T}}^{\text {miss }}$ measurements are affected dominantly by the lepton resolutions, as described in Sec. II. Multiplying $A$ with the theoretical cross section $\sigma$ and the branching fraction $\mathcal{B}$, the result can be compared with an exclusion limit from Fig. 10 or 11. Values of $\sigma \times \mathcal{B} \times A$ larger than the limit indicated by the solid line can be excluded. To find the best value of $M_{\mathrm{T}}^{\min }$, the threshold should be optimized with respect to the expected limit.

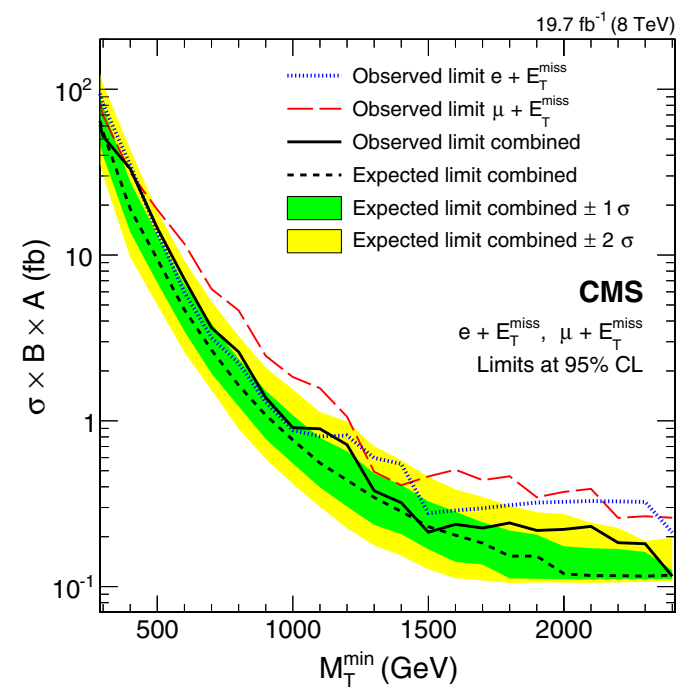

FIG. 11 (color online). Cross section upper limits at 95\% on the effective cross section $\sigma\left(W^{\prime}\right) \mathcal{B}\left(W^{\prime} \rightarrow \ell \nu\right) A$ above a threshold $M_{\mathrm{T}}^{\min }$ for the combination of the electron and muon channels. Shown are the observed limits of the electron channel, muon channel, and the combination of both channels and the combined expected limit, together with the combined expected limit $1 \sigma$ and $2 \sigma$ intervals. Only detector acceptance is taken into account for the signal. The parameter $A$ describes the efficiency derived from the kinematic selection criteria and the $M_{\mathrm{T}}^{\min }$ threshold. 
The electron channel is more sensitive than the muon channel, as the energy resolution is superior. In the muon channel, shown in Fig. 10 (bottom), a small excess of events yields a larger and therefore worse observed limit for most values of $M_{\mathrm{T}}^{\min }$ compared to the expectation. The steplike structure for high transverse mass thresholds corresponds to the small discrete numbers of events in these regions.

\section{B. Limits on an SSM $W^{\prime}$-boson}

The search for an SSM $W^{\prime}$-boson yields limits on the cross section times branching fraction for the electron and muon channels. The multibin method is used to determine the 95\% upper cross section limits, as shown in Fig. 12. The indicated theoretical cross sections are the NNLO values for the lepton $+E_{\mathrm{T}}^{\text {miss }}$ channel, as detailed in Sec. III, and
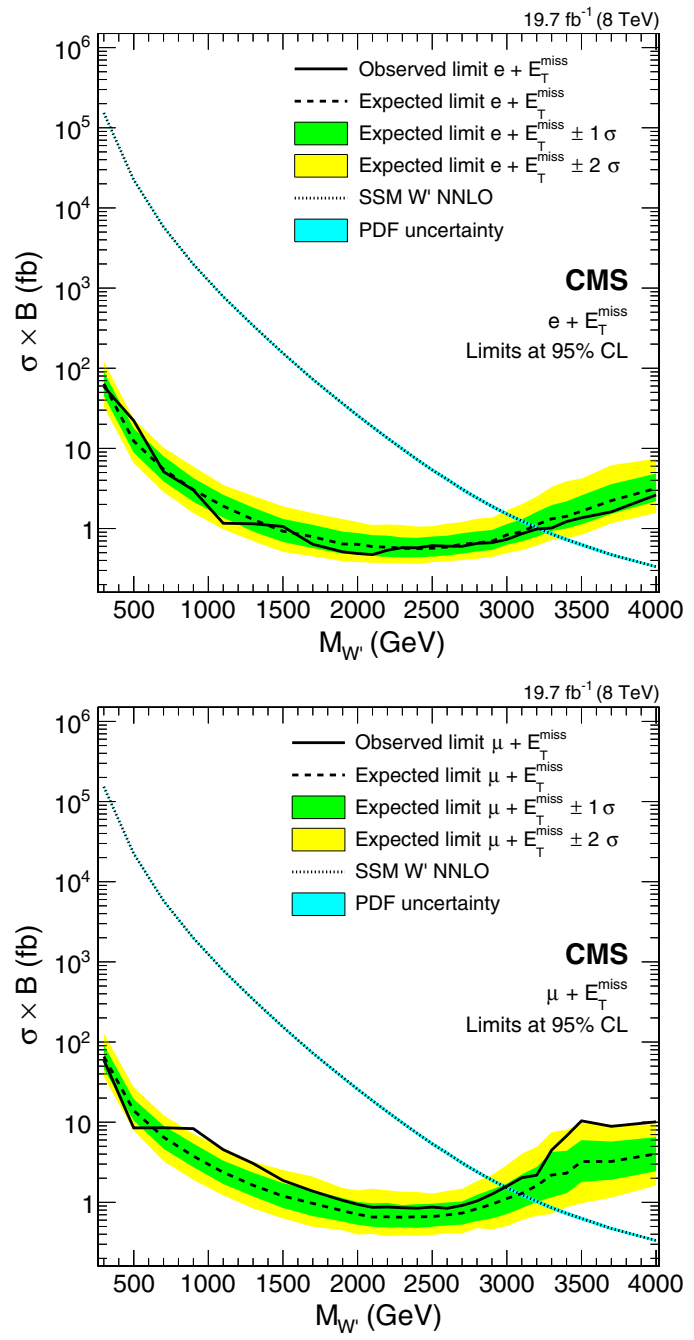

FIG. 12 (color online). Upper limits at 95\% C.L. on $\sigma\left(W^{\prime}\right) \mathcal{B}\left(W^{\prime} \rightarrow \ell \nu\right)$ with $\ell=e$ (top) and $\ell=\mu$ (bottom). Shown are the theoretical cross section, the observed limit, the expected limit, and the expected limit $1 \sigma$ and $2 \sigma$ intervals. The theoretical cross section incorporates a mass-dependent NNLO K-factor. are the same for both channels. The PDF uncertainties are shown as a thin band around the NNLO cross section. The central value of the theoretical cross section times branching fraction is used for deriving the mass limit. The existence of an SSM $W^{\prime}$-boson of mass less than $3.22 \mathrm{TeV}$ (compared with an expected limit of $3.18 \mathrm{TeV}$ ) in the electron channel, and $2.99 \mathrm{TeV}$ (compared with an expected $3.09 \mathrm{TeV}$ ) in the muon channel, is excluded. The electron channel has a slightly higher expected sensitivity because of its better resolution.

Limits can also be obtained for the combined electron and muon channels. Uncertainties deriving from the lepton identification efficiencies for each channel are assumed to be independent. Uncertainties due to the $E_{\mathrm{T}}^{\text {miss }}$ determination, pileup estimate, and luminosity measurement are each assumed to be fully correlated between the channels. Combining both channels, which corresponds to doubling the event count, increases the mass limit to $3.28 \mathrm{TeV}$ (compared with an expected limit of $3.26 \mathrm{TeV}$ ). This compares with the previously established combined limit of $2.5 \mathrm{TeV}$ [23], which is based on an integrated luminosity of $5 \mathrm{fb}^{-1}$ at $\sqrt{s}=7 \mathrm{TeV}$. Figure 13 displays the excluded $W^{\prime}$ cross section times branching ratio as a function of the $W^{\prime}$-boson mass. The corresponding values are summarized in Table VI.

If the cross section limits are compared to the LO cross section, the SSM $W^{\prime}$ mass limits change slightly to $3.16 \mathrm{TeV}$ (compared with an expected limit of $3.14 \mathrm{TeV}$ )

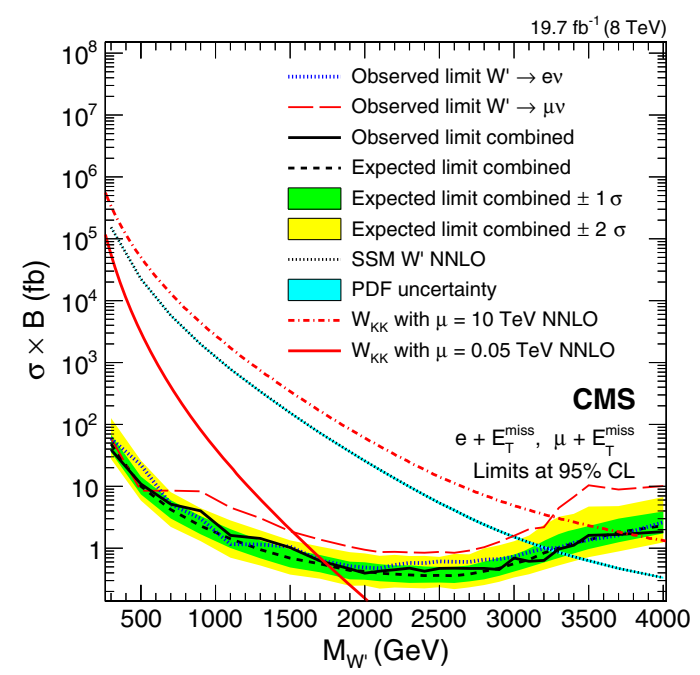

FIG. 13 (color online). Limits for heavy $W^{\prime}$-bosons for the electron and the muon channels, and for the two channels combined. Shown are the theoretical cross section for the SSM and two split-UED scenarios, the observed limit, the expected limit, and the expected limit $1 \sigma$ and $2 \sigma$ intervals. The theoretical cross sections incorporate a mass-dependent NNLO K-factor. The PDF uncertainties for the SSM are shown as a light blue band around the cross section curve. For the splitUED scenarios, the PDF uncertainties are expected to be small, similar to the uncertainty in the SSM scenario. 
TABLE VI. Upper cross section limits at $95 \%$ for various SSM $W^{\prime}$-boson masses, based on the combination of the electron and muon channels.

\begin{tabular}{lccccccc}
\hline \hline$M_{W^{\prime}}(\mathrm{GeV})$ & 300 & 1100 & 1500 & 2000 & 3000 & 3500 & 4000 \\
\hline Expected limit (fb) & 48 & 1.4 & 0.69 & 0.42 & 0.56 & 1.4 & 2.3 \\
Observed limit (fb) & 39 & 1.6 & 1 & 0.4 & 0.69 & 1.6 & 1.9 \\
\hline \hline
\end{tabular}

for the electron channel, $2.96 \mathrm{TeV}$ (compared with an expected limit of $3.04 \mathrm{TeV}$ ) for the muon channel, and $3.25 \mathrm{TeV}$ (compared with an expected limit of $3.21 \mathrm{TeV}$ ) for the combination of both channels.

\section{Interpretation in the HNC-CI model}

Another interpretation of the observed data can be made in the framework of the HNC-CI model, providing a limit on the contact interaction scale $\Lambda$. The statistical interpretation is identical to that for an SSM $W^{\prime}$-boson, using a Bayesian multibin approach with a uniform prior for the signal cross section [56]. The difference in shape with respect to the $W^{\prime}$ Jacobian peak does not affect the limitsetting procedure. The cross section scales as $\Lambda^{-4}$. The shape and signal efficiency, however, are independent of $\Lambda$, leading to the constant expected and observed limits shown in Fig. 14. The limit on $\Lambda$ is calculated to be $11.3 \mathrm{TeV}$ in the electron and $10.9 \mathrm{TeV}$ in the muon channel. In the considered model, the contact interaction must be flavor symmetric for $\Lambda<500 \mathrm{TeV}$ [2]. Combining both channels, a limit on $\Lambda$ of $12.4 \mathrm{TeV}$ is observed, and $13.6 \mathrm{TeV}$ is expected.

\section{Dark matter interpretation}

The data may be interpreted in the context of an effective dark matter theory. The search presented is inclusive, meaning that it includes final states of lepton $+E_{\mathrm{T}}^{\text {miss }}$ and well as lepton $+E_{\mathrm{T}}^{\text {miss }}+$ jet, since no event selection criteria based on jets are applied. The statistical interpretation is a Bayesian approach with a uniform prior [56], based on the multibin approach. To be comparable to other dark matter searches, the limits in this model are determined at 90\% C.L. Electron and muon channels are combined, since the recoiling $W$-boson is a standard model boson, for which the decay channel should not depend on the new physics model. Vectorlike (spinindependent) and axial-vector-like (spin-dependent) couplings are considered.

The exclusion limits are determined as cross section limits (see Fig. 15) which are subsequently transformed into limits on the effective scale parameter $\Lambda$ as a function of $M_{\chi}$, as shown in Fig. 16. To compare these collider limits with results from direct detection experiments, they are translated into limits on the DM-proton cross section, shown in Fig. 17. We recalculate the excluded nucleon
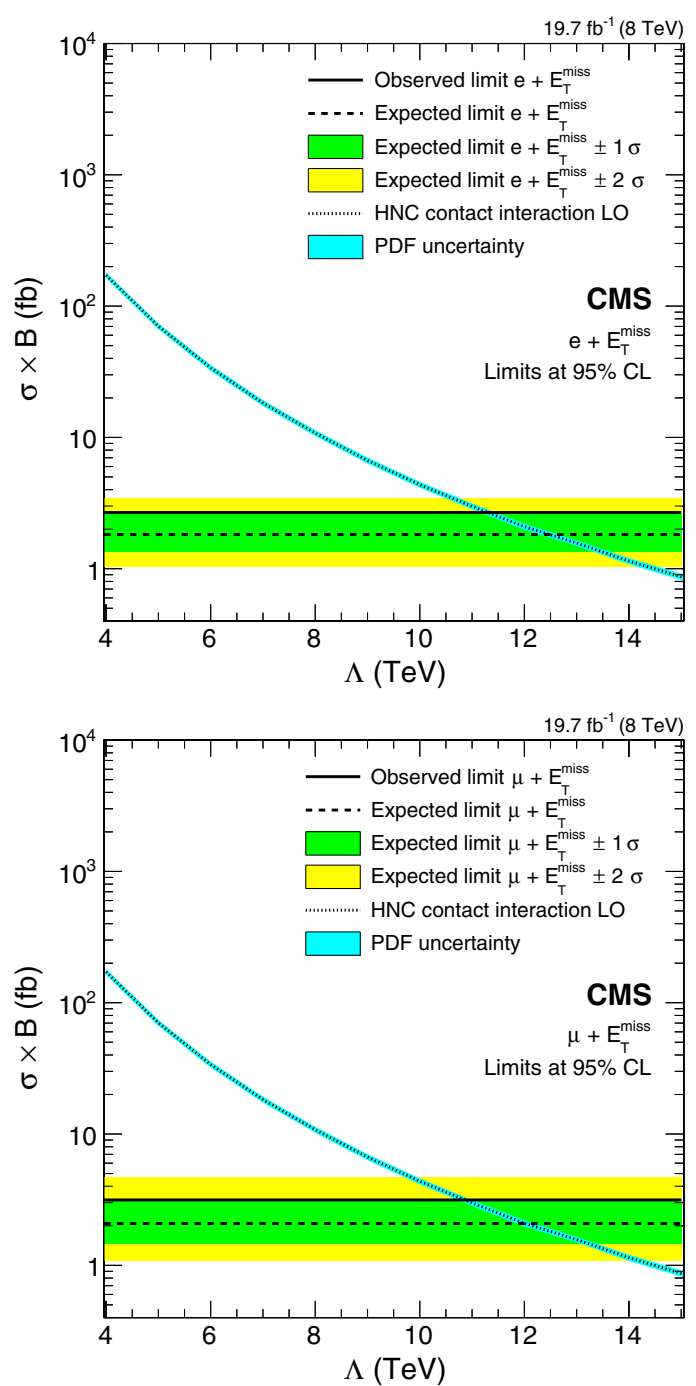

FIG. 14 (color online). Upper limits at $95 \%$ on $\sigma \mathcal{B}(p p \rightarrow \ell \nu)$ with $\ell=e$ (top) and $\ell=\mu$ (bottom) in terms of the contact interaction scale $\Lambda$ in the HNC-CI model.

cross section for a given $\Lambda$ and $M_{\chi}$ using the conversion formula with interference from Ref. [86].

The collider cross section limits in Fig. 15 show that the excluded cross section is flat as a function of $M_{\chi}$, as expected since the signal kinematics do not change appreciably for different $M_{\chi}$. The coupling does not have a large effect on the excluded cross section. The different interference scenarios have a visible influence on the limit. In the case of $\xi=+1$, a cross section greater than $0.6 \mathrm{pb}$ is excluded, whereas for $\xi=0$ and for $\xi=-1$, the cross section limit is $0.05 \mathrm{pb}$. For high $M_{\chi}$ the phase space to produce two heavy particles and a $W$-boson is small, and therefore the signal cross section is reduced, and its shape is more consistent with the background. These effects yield a reduced sensitivity at this center-of-mass energy.

As the $M_{\mathrm{T}}$ distributions for vectorlike (spin-independent) and axial-vector-like (spin-dependent) couplings are very 

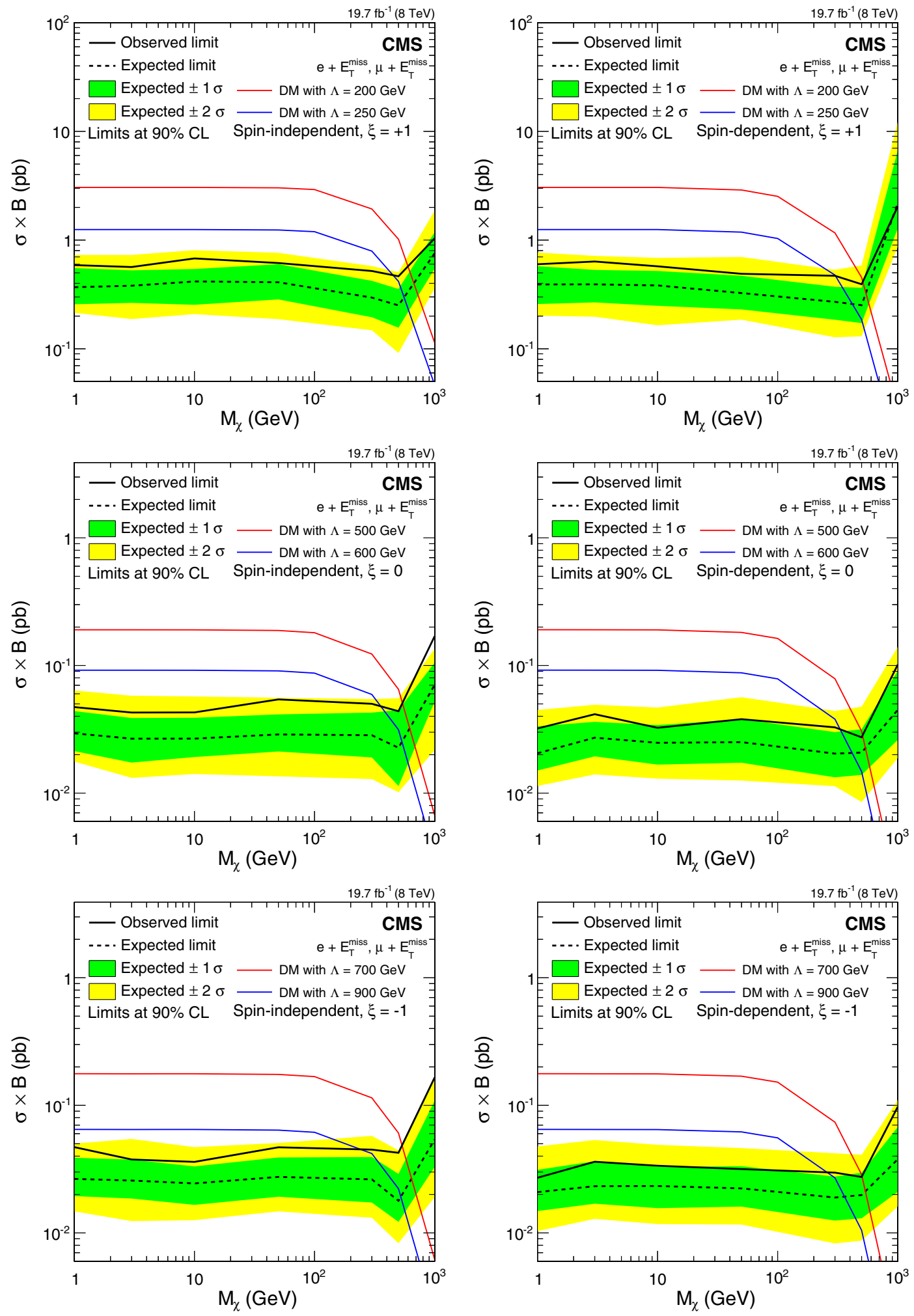

FIG. 15 (color online). Upper limits on $\sigma \mathcal{B}(p p \rightarrow \chi \chi \ell \nu)$ for vectorlike (spin-independent, left column) and axial-vector-like (spindependent, right column) couplings. Limits are calculated for the cases (from top to bottom) $\xi=+1,0$ and -1 . Note the different vertical scales. 
similar, the derived limits for the two cases do not differ substantially.

For lower masses a constant $\Lambda$ exclusion is obtained for $M_{\chi} \leq 100 \mathrm{GeV}$ of $\Lambda<300 \mathrm{GeV}$ for $\xi=+1, \quad \Lambda<$ $700 \mathrm{GeV}$ for $\xi=0$, and $\Lambda<1000 \mathrm{GeV}$ for $\xi=-1$. The difference between vectorlike and axial-vector-like couplings is small for low $M_{\chi}$ for all three values of $\xi$, but a difference is observed in the high- $M_{\chi}$ region, above $100 \mathrm{GeV}$. An overview is given in Fig. 16.

For comparison the limit from the monojet final state [53] is shown. The conversion to the $\chi$-proton cross section depends on the coupling parameter $\xi$, although the monojet
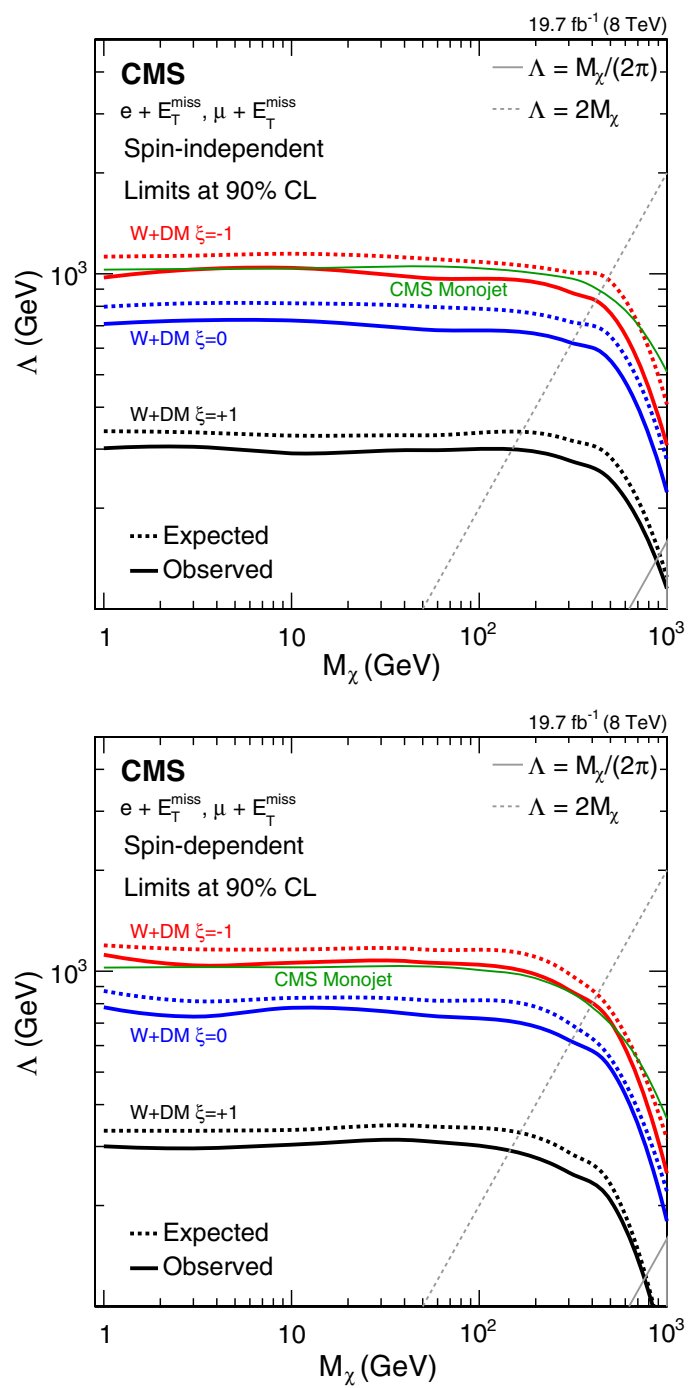

FIG. 16 (color online). Exclusion plane in $\Lambda-M_{\chi}$, for the combination of the electron and muon channels. Vectorlike (top) and axial-vector-like (bottom) couplings are shown. The two gray lines indicate where the coupling becomes nonperturbative and $\left(g_{\mathrm{DM}}\right)$ is equal to 1 , as described in Sec. III C. The green line shows the limit in the monojet final state [53], which is independent of $\xi$ for the limit on $\Lambda$.
TABLE VII. The $\chi$-proton cross section upper limits at $90 \%$ for $M_{\chi}=10 \mathrm{GeV}$.

\begin{tabular}{lcc}
\hline$\xi$ & Vector coupling $\left(\mathrm{cm}^{2}\right)$ & Axial-vector coupling $\left(\mathrm{cm}^{2}\right)$ \\
\hline-1 & $4 \times 10^{-41}$ & $1 \times 10^{-40}$ \\
0 & $6 \times 10^{-40}$ & $2 \times 10^{-40}$ \\
+1 & $3 \times 10^{-38}$ & $2 \times 10^{-39}$ \\
\hline
\end{tabular}

analysis is not sensitive to $\xi$. The limits determined from the direct detection experiments depend on different model assumptions [87]. Therefore, we do not give a direct comparison here.

The $\chi$-proton cross section upper limits at $90 \%$ for $M_{\chi}=$ $10 \mathrm{GeV}$ are presented in Table VII and Fig. 17.
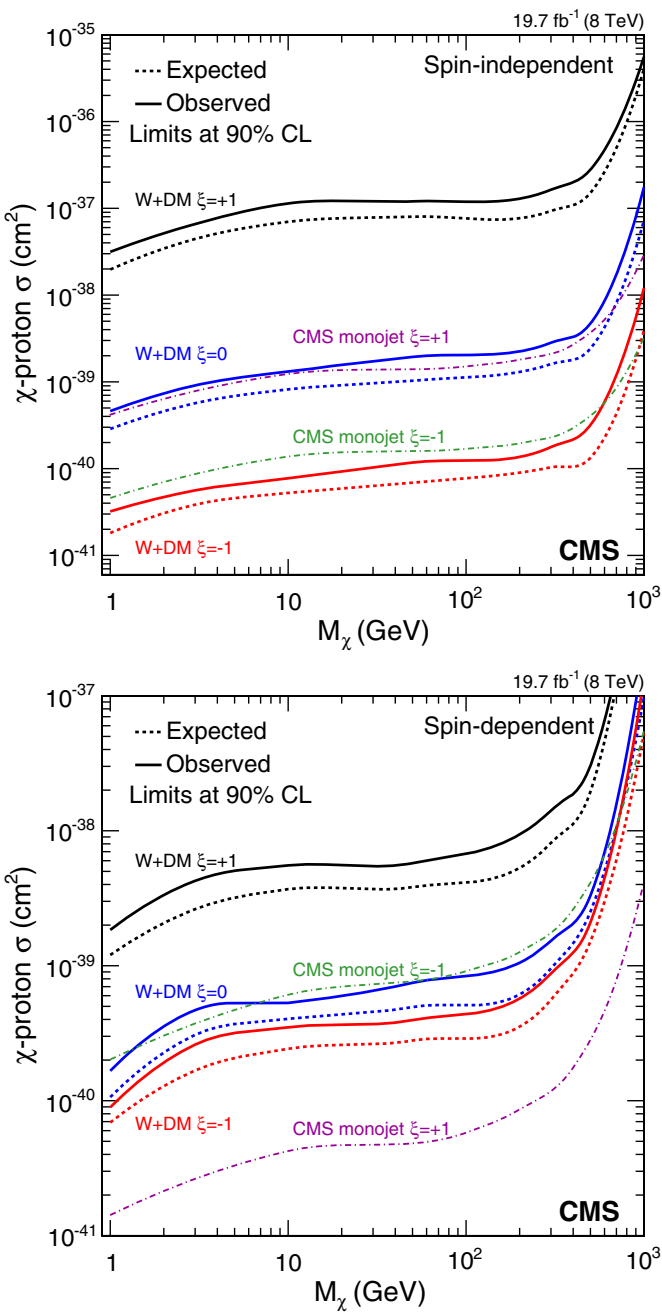

FIG. 17 (color online). Excluded proton-dark matter cross section for vectorlike (top) and axial-vector-like (bottom) couplings, for the combination of the electron and muon channels. For comparison the result from the monojet DM search [53] is also shown. 

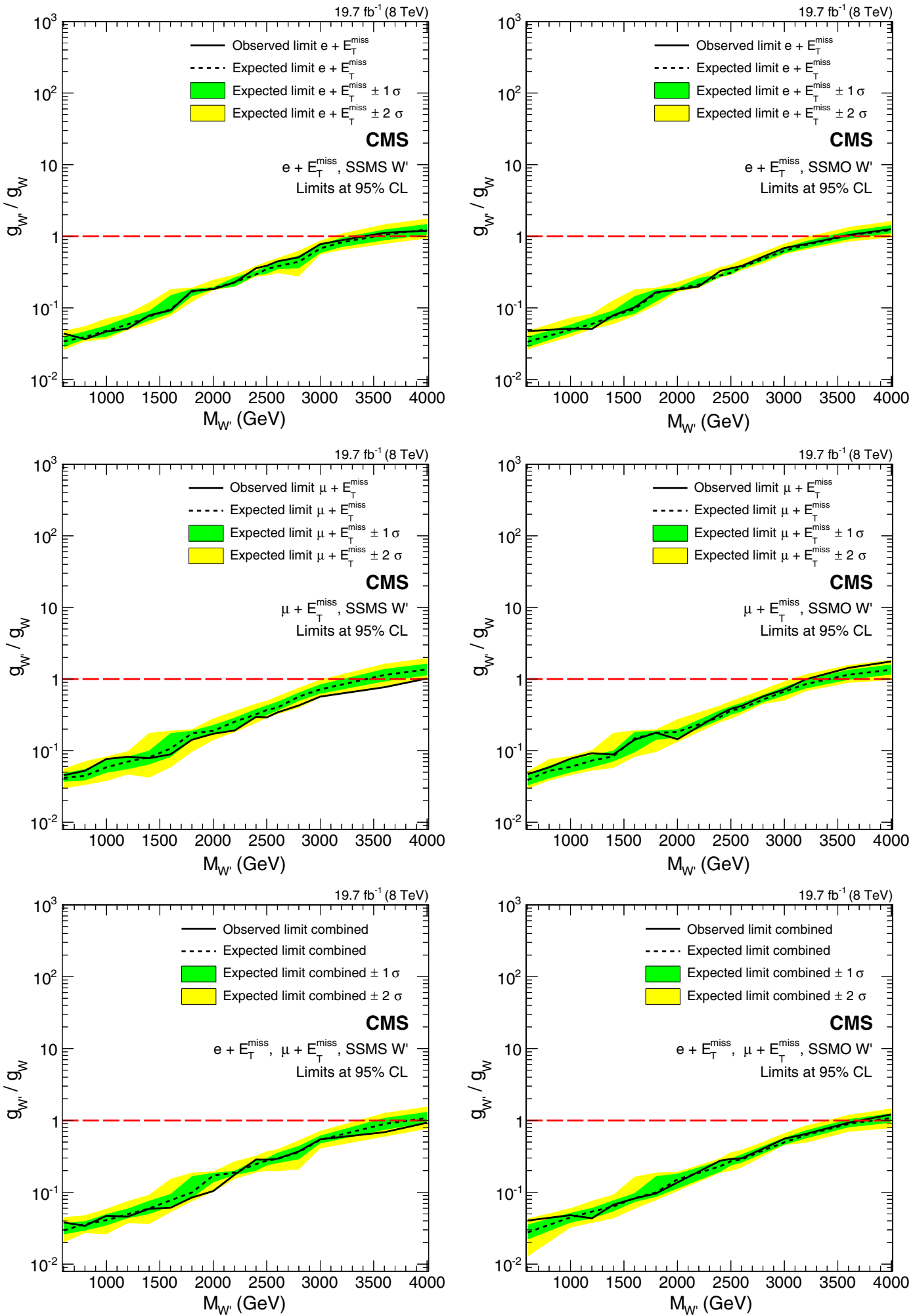

FIG. 18 (color online). Limits on the coupling $g_{W^{\prime}}$ in terms of the SM coupling $g_{W}$ in the electron (top row) and muon (middle row) channels and their combination (bottom row). Limits for the SSMS model are displayed in the left column, and those for the SSMO model are in the right column. 


\section{E. Limits on coupling strength in models with interference}

Limits on the $W^{\prime}$-boson models, taking into account interference effects with the $W$-boson, are set on the $W^{\prime}$ coupling $g_{W^{\prime}}$ in terms of the SM $W$-boson coupling strength $g_{W}$. The corresponding impact on the observable distribution is modeled using a reweighting technique. Thus, effects such as the influence on the decay width and the impact on interference by the altered coupling are taken into account, as shown in Fig. 3 (middle right). The following interference occurs in the mass range between $M_{W}-$ and $M_{W^{\prime}}$. If the coupling of the $W^{\prime}$-boson has the same sign (SSMS) with respect to the $W$-boson coupling to left-handed fermions, the interference effect is destructive; in case of opposite sign (SSMO) coupling, it is constructive. For $M_{\mathrm{T}}>M_{W^{\prime}}$ the effect is vice versa.

The limit on the coupling strength $g_{W^{\prime}}$ as a function of the $W^{\prime}$-boson mass is shown in Fig. 18. The mass limits in the case where the $W^{\prime}$-boson coupling is equal to the $W$-boson coupling are summarized in Table VIII.

\section{F. Interpretation in the split-UED model}

The observed limits on the SSM $W^{\prime}$-boson (see Sec. IX B) can be reinterpreted as limits on the $W_{\mathrm{KK}}^{(2)}$ mass in the framework of split-UED, with the second KK excitation being the only accessible state at present LHC energies with nonzero couplings to SM particles. Figure 13 shows two examples of $W_{\mathrm{KK}}^{(2)}$-boson mass limits for values of the bulk mass parameter $\mu=0.05 \mathrm{TeV}$ and $\mu=10 \mathrm{TeV}$. For these two examples, the lower mass limit is $1.74 \mathrm{TeV}$ for $\mu=$ $0.05 \mathrm{TeV}$ and $3.71 \mathrm{TeV}$ for $\mu=10 \mathrm{TeV}$, when combining both channels. The lower limits on the mass can be directly translated into bounds on the split-UED parameter space $(1 / R, \mu)$ as shown in Fig. 19.

\section{G. Interpretation in the $\mathbf{T e V}^{-1}$ model}

Based on the model-independent cross section limit and the recipe described in Sec. IX A, a single-bin limit on the compactification scale $M_{\mathrm{C}}$ of the $\mathrm{TeV}^{-1}$ model is derived using the kinematic selection efficiency for this particular

TABLE VIII. Summary of all SSMS and SSMO exclusion limits on the $W^{\prime}$-boson mass in the electron and muon channels and for their combination assuming $g_{W}=g_{W^{\prime}}$.

\begin{tabular}{lccc}
\hline \hline Model & Channel & $\begin{array}{c}\text { Observed lower } \\
\text { limit }(\mathrm{TeV})\end{array}$ & $\begin{array}{c}\text { Expected lower } \\
\text { limit }(\mathrm{TeV})\end{array}$ \\
\hline SSMS & $e$ & 3.41 & 3.52 \\
SSMS & $\mu$ & 3.97 & 3.43 \\
SSMS & Combined & 4.00 & 3.83 \\
SSMO & $e$ & 3.54 & 3.57 \\
SSMO & $\mu$ & 3.22 & 3.38 \\
SSMO & Combined & 3.71 & 3.83 \\
\hline \hline
\end{tabular}

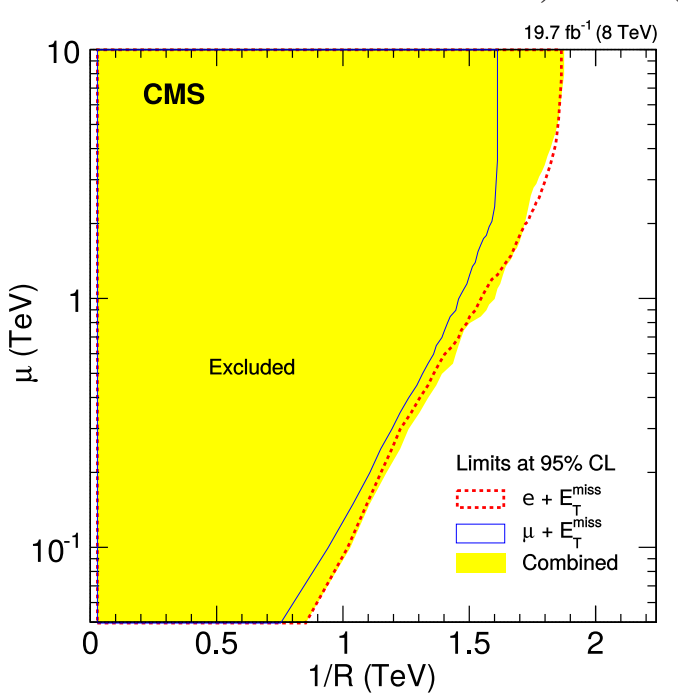

FIG. 19 (color online). Limits on the split-UED parameters $\mu$ and $1 / R$, derived from the $W^{\prime}$-boson mass limits, taking into account the corresponding width of the $W_{\mathrm{KK}}^{(2)}$-boson.

model. The lower bound on $M_{\mathrm{C}}$ is established as $3.4 \mathrm{TeV}$. The existing indirect limit on $M_{\mathrm{C}}$ is $6.8 \mathrm{TeV}$, which was obtained by fitting results from LEP2, Tevatron, and HERA experiments [88]. The lower limit set by LEP2 experiments is $6.6 \mathrm{TeV}$, which is the dominant contribution. The sensitivity of this analysis is therefore still less stringent than that based on LEP2 data.

\section{SUMMARY}

A search for physics beyond the standard model, based on events with a final state containing a charged lepton (electron or muon) and significant missing transverse energy, has been performed, using proton-proton collision data at $\sqrt{s}=8 \mathrm{TeV}$, corresponding to an integrated luminosity of $19.7 \mathrm{fb}^{-1}$. No significant deviation from the standard model expectation has been observed in the transverse mass distribution.

A model-independent upper limit at $95 \%$ on the cross section times branching fraction of additional contributions has been established, ranging from 100 to $0.1 \mathrm{fb}$ over an $M_{\mathrm{T}}$ range of $300 \mathrm{GeV}$ to $2.5 \mathrm{TeV}$, respectively. The results have been interpreted in the context of various models, as summarized below.

A SSM $W^{\prime}$-boson that does not interfere with the $W$ boson has been excluded at $95 \%$ for $W^{\prime}$-boson masses up to 3.22 (2.99) $\mathrm{TeV}$ for the electron (muon) channel, where the expected limit is 3.18 (3.06) TeV. When combining both channels, the limit improves to $3.28 \mathrm{TeV}$. Lower mass limits in either channel are implicit due to trigger thresholds.

An interpretation in terms of a four-fermion contact interaction yields a limit for the compositeness energy scale $\Lambda$ of $11.3 \mathrm{TeV}$ in the electron channel, $10.9 \mathrm{TeV}$ in the muon channel, and $12.4 \mathrm{TeV}$ for their combination. 
Assuming the production of a pair of dark matter particles along with a recoiling $W$-boson that subsequently decays leptonically, the results have been reinterpreted in terms of an effective dark matter theory. The effective scale is excluded below 0.3 to $1 \mathrm{TeV}$, depending on model parameters. This is particularly interesting for low masses of dark matter particles, where the sensitivity of direct searches is poor.

Building upon earlier versions of this analysis [24], the expected impact of $W--W^{\prime}$ interference on the shape of the $W$-boson $M_{\mathrm{T}}$ distribution is fully taken into account. Along with the shape, the expected cross section varies, making possible the setting of limits for models with both destructive (SSMS) and constructive (SSMO) interference.
The lower limit on the $W^{\prime}$-boson mass is 3.41 (3.97) TeV in the electron (muon) channels for the SSMS and 3.54 (3.22) $\mathrm{TeV}$ for the SSMO. For the first time, limits in terms of generalized lepton couplings are given.

An interpretation of the search results has been made in a specific framework of universal extra dimensions where bulk fermions propagate in the one additional dimension. The second Kaluza-Klein excitation $W_{\mathrm{KK}}^{(2)}$ has been excluded for masses below $1.74 \mathrm{TeV}$, assuming a bulk mass parameter $\mu$ of $0.05 \mathrm{TeV}$, or for masses below $3.71 \mathrm{TeV}$, for $\mu=10 \mathrm{TeV}$. In an alternative model in which only SM gauge bosons propagate in a compactified extra dimension $\left(\mathrm{TeV}^{-1}\right.$ model), a lower bound on the size of the compactified dimension, $M_{C}$, has been set at $3.40 \mathrm{TeV}$.

\section{CMS}

$19.7 \mathrm{fb}^{-1}(8 \mathrm{TeV})$

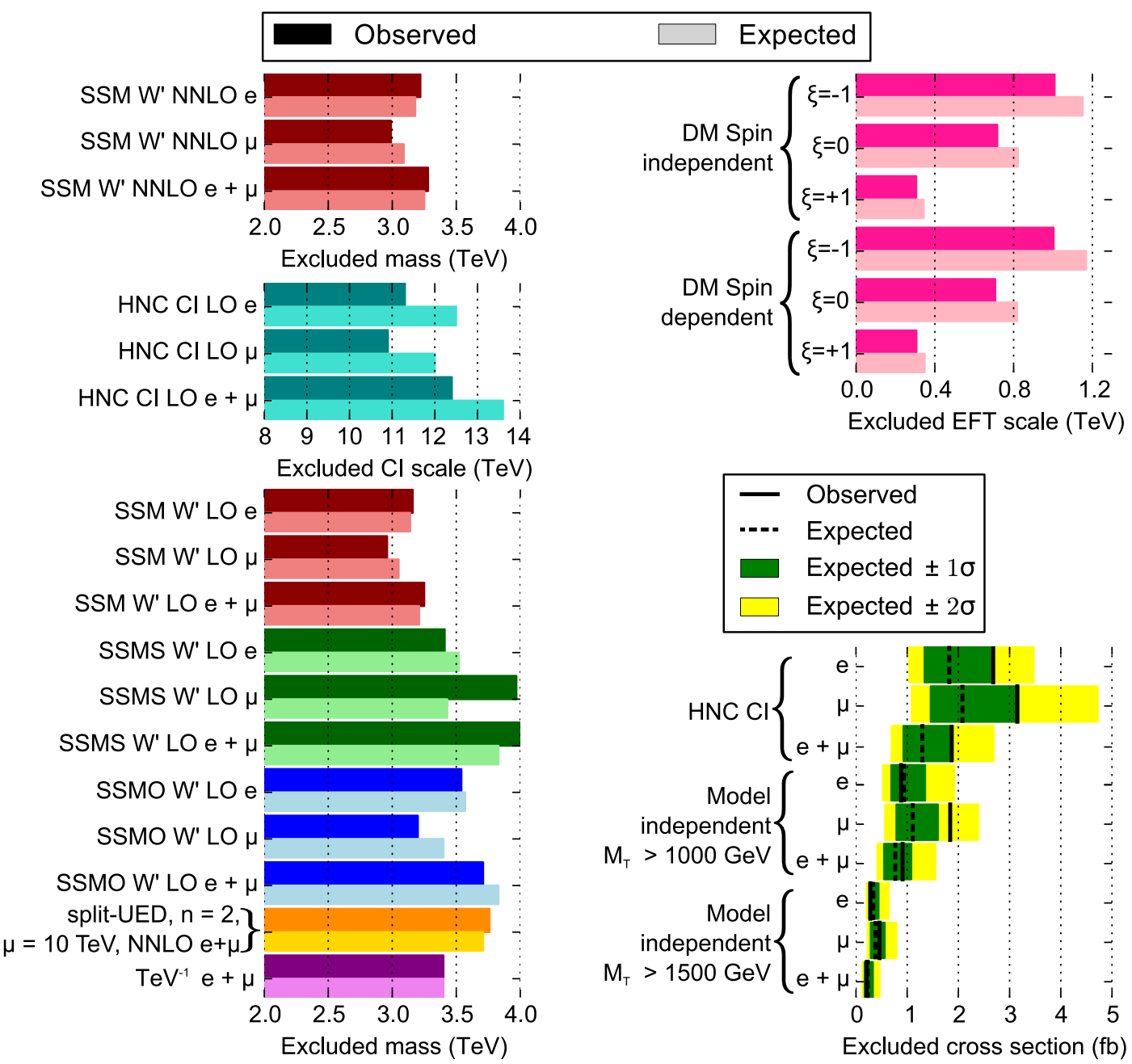

FIG. 20 (color online). Summary of all exclusion limits in the electron and muon channels and for their combinations. No interference of $W^{\prime}$ - and $W$-bosons is considered in the interpretation labeled as SSM, while it is taken into account in the SSMS and SSMO models. For the HNC contact interaction, the compositeness scale $\Lambda$ is probed. In the upper rows of the right column, the EFT limits are shown for the DM interpretations in term of $\Lambda$ for small dark matter masses $M_{\chi}<100 \mathrm{GeV}$. The reinterpretation in terms of additional extra dimensions is provided in the context of split-UED, given for a bulk mass parameter $\mu=10 \mathrm{TeV}$, and in the $\mathrm{TeV}^{-1} \mathrm{model}$ 
Study of the monolepton channel provides a powerful tool to probe for beyond the standard model physics. All the results of this search are summarized in Fig. 20, including the expected and observed limits. Figure 20 is structured by theories and the related model parameters (particle mass, compositeness scale $\Lambda$, or dark matter effective field scale $\Lambda$ ) as given in Table I. The three representative signal examples (SSM $W^{\prime}$, contact interaction, and dark matter) are shown in the upper figure section. Limits for specific models along with the model-independent cross section limit are given in the lower part of the figure.

\section{ACKNOWLEDGMENTS}

We congratulate our colleagues in the CERN accelerator departments for the excellent performance of the LHC and thank the technical and administrative staffs at CERN and at other CMS institutes for their contributions to the success of the CMS effort. In addition, we gratefully acknowledge the computing centers and personnel of the Worldwide LHC Computing Grid for delivering so effectively the computing infrastructure essential to our analyses. Finally, we acknowledge the enduring support for the construction and operation of the LHC and the CMS detector provided by the following funding agencies: the Austrian Federal Ministry of Science, Research and Economy and the Austrian Science Fund; the Belgian Fonds de la Recherche Scientifique, and Fonds voor Wetenschappelijk Onderzoek; the Brazilian Funding Agencies (CNPq, CAPES, FAPERJ, and FAPESP); the Bulgarian Ministry of Education and Science; CERN; the Chinese Academy of Sciences, Ministry of Science and Technology and National Natural Science Foundation of China; the Colombian Funding Agency (COLCIENCIAS); the Croatian Ministry of Science, Education and Sport and the Croatian Science Foundation; the Research Promotion Foundation, Cyprus; the Ministry of Education and Research, Estonian Research Council via IUT23-4 and IUT23-6 and European Regional Development Fund, Estonia; the Academy of Finland, Finnish Ministry of Education and Culture, and Helsinki Institute of Physics; the Institut National de Physique Nucléaire et de Physique des Particules/CNRS and Commissariat à l'Énergie Atomique et aux Énergies Alternatives/CEA, France; the Bundesministerium für Bildung und Forschung, Deutsche Forschungsgemeinschaft, and Helmholtz-Gemeinschaft Deutscher Forschungszentren, Germany; the General Secretariat for Research and Technology, Greece; the National Scientific Research Foundation and National Innovation Office, Hungary; the Department of Atomic Energy and the Department of Science and Technology, India; the Institute for Studies in Theoretical Physics and
Mathematics, Iran; the Science Foundation, Ireland; the Istituto Nazionale di Fisica Nucleare, Italy; the Korean Ministry of Education, Science and Technology and the World Class University program of NRF, Republic of Korea; the Lithuanian Academy of Sciences; the Ministry of Education and University of Malaya, Malaysia; the Mexican Funding Agencies (CINVESTAV, CONACYT, SEP, and UASLP-FAI); the Ministry of Business, Innovation and Employment, New Zealand; the Pakistan Atomic Energy Commission; the Ministry of Science and Higher Education and the National Science Centre, Poland; the Fundação para a Ciência e a Tecnologia, Portugal; JINR, Dubna; the Ministry of Education and Science of the Russian Federation, the Federal Agency of Atomic Energy of the Russian Federation, Russian Academy of Sciences, and the Russian Foundation for Basic Research; the Ministry of Education, Science and Technological Development of Serbia; the Secretaría de Estado de Investigación, Desarrollo e Innovación and Programa Consolider-Ingenio 2010, Spain; the Swiss Funding Agencies (ETH Board, ETH Zurich, PSI, SNF, UniZH, Canton Zurich, and SER); the Ministry of Science and Technology, Taiwan; the Thailand Center of Excellence in Physics, the Institute for the Promotion of Teaching Science and Technology of Thailand, Special Task Force for Activating Research, and the National Science and Technology Development Agency of Thailand; the Scientific and Technical Research Council of Turkey and Turkish Atomic Energy Authority; the National Academy of Sciences of Ukraine and State Fund for Fundamental Researches, Ukraine; the Science and Technology Facilities Council, United Kingdom; and the US Department of Energy and the US National Science Foundation. Individuals have received support from the Marie-Curie Program and the European Research Council and EPLANET (European Union); the Leventis Foundation; the A.P. Sloan Foundation; the Alexander von Humboldt Foundation; the Belgian Federal Science Policy Office; the Fonds pour la Formation à la Recherche dans l'Industrie et dans l'Agriculture (Belgium); the Agentschap voor Innovatie door Wetenschap en Technologie (Belgium); the Ministry of Education, Youth and Sports of the Czech Republic; the Council of Science and Industrial Research, India; the HOMING PLUS program of Foundation for Polish Science, cofinanced from the European Union, Regional Development Fund; the Compagnia di San Paolo (Torino); the Consorzio per la Fisica (Trieste); MIUR Project No. 20108T4XTM (Italy); the Thalis and Aristeia programs cofinanced by EU-ESF and the Greek NSRF; and the National Priorities Research Program by Qatar National Research Fund. 
[1] G. Altarelli, B. Mele, and M. Ruiz-Altaba, Searching for new heavy vector bosons in $p \bar{p}$ colliders, Z. Phys. C 45, 109 (1989).

[2] K. D. Lane, F. E. Paige, T. Skwarnicki, and W. J. Womersley, Simulations of supercollider physics, Phys. Rep. 278, 291 (1997).

[3] J. Goodman, M. Ibe, A. Rajaraman, W. Shepherd, T. M. P. Tait, and H.-B. Yu, Constraints on light Majorana dark matter from colliders, Phys. Lett. B 695, 185 (2011).

[4] J. Goodman, M. Ibe, A. Rajaraman, W. Shepherd, T. M. P. Tait, and H.-B. Yu, Constraints on dark matter from colliders, Phys. Rev. D 82, 116010 (2010).

[5] M. Beltran, D. Hooper, E. W. Kolb, and Z. A. C. Krusberg, Deducing the nature of dark matter from direct and indirect detection experiments in the absence of collider signatures of new physics, Phys. Rev. D 80, 043509 (2009).

[6] E. Accomando, D. Becciolini, S. De Curtis, D. Dominici, L. Fedeli, and C. Shepherd-Themistocleous, Interference effects in heavy $\mathrm{W}^{\prime}$-boson searches at the LHC, Phys. Rev. D 85, 115017 (2012).

[7] T. G. Rizzo, The determination of the helicity of $W^{\prime}$ boson couplings at the LHC, J. High Energy Phys. 05 (2007) 037.

[8] E. Boos, V. Bunichev, L. Dudko, and M. Perfilov, Interference between $W^{\prime}$ and $W$ in single-top quark production processes, Phys. Lett. B 655, 245 (2007).

[9] C.-R. Chen, M. M. Nojiri, S. Chan Park, J. Shu, and M. Takeuchi, Dark matter and collider phenomenology of split-UED, J. High Energy Phys. 09 (2009) 078.

[10] K. Kong, S. C. Park, and T. G. Rizzo, Collider phenomenology with Split-UED, J. High Energy Phys. 04 (2010) 081.

[11] K. R. Dienes, E. Dudas, and T. Gherghetta, Grand unification at intermediate mass scales through extra dimensions, Nucl. Phys. B537, 47 (1999).

[12] A. Pomarol and M. Quiros, The standard model from extra dimensions, Phys. Lett. B 438, 255 (1998).

[13] M. Masip and A. Pomarol, Effects of standard model Kaluza-Klein excitations on electroweak observables, Phys. Rev. D 60, 096005 (1999).

[14] I. Antoniadis, K. Benakli, and M. Quiros, Direct collider signatures of large extra dimensions, Phys. Lett. B 460, 176 (1999).

[15] V. M. Abazov et al. (D0 Collaboration), Search for $W^{\prime}$ bosons decaying to an electron and a neutrino with the D0 detector, Phys. Rev. Lett. 100, 031804 (2008).

[16] T. Aaltonen et al. (CDF Collaboration), Search for a new heavy gauge boson $W^{\prime}$ with event signature electron + missing transverse energy in $p \bar{p}$ collisions at $\sqrt{s}=1.96 \mathrm{TeV}$, Phys. Rev. D 83, 031102 (2011).

[17] ATLAS Collaboration, Search for high-mass states with one lepton plus missing transverse momentum in proton-proton collisions at $\sqrt{s}=7 \mathrm{TeV}$ with the ATLAS detector, Phys. Lett. B 701, 50 (2011).

[18] ATLAS Collaboration, Search for a heavy gauge boson decaying to a charged lepton and a neutrino in $1 \mathrm{fb}^{-1}$ of $\mathrm{pp}$ collisions at $\sqrt{s}=7 \mathrm{TeV}$ using the ATLAS detector, Phys. Lett. B 705, 28 (2011).

[19] ATLAS Collaboration, ATLAS search for a heavy gauge boson decaying to a charged lepton and a neutrino in pp collisions at $\sqrt{s}=7 \mathrm{TeV}$, Eur. Phys. J. C 72, 2241 (2012).

[20] ATLAS Collaboration, Search for new particles in events with one lepton and missing transverse momentum in $p p$ collisions at $\sqrt{s}=8 \mathrm{TeV}$ with the ATLAS detector, J. High Energy Phys. 09 (2014) 037.

[21] CMS Collaboration, Search for a heavy gauge boson $\mathrm{W}^{\prime}$ in the final state with an electron and large missing transverse energy in pp collisions at $\sqrt{s}=7 \mathrm{TeV}$, Phys. Lett. B 698, 21 (2011).

[22] CMS Collaboration, Search for a $W^{\prime}$ boson decaying to a muon and a neutrino in pp collisions at $\sqrt{s}=7 \mathrm{TeV}$, Phys. Lett. B 701, 160 (2011).

[23] CMS Collaboration, Search for leptonic decays of $\mathrm{W}^{\prime}$ bosons in pp collisions at $\sqrt{s}=7 \mathrm{TeV}$, J. High Energy Phys. 08 (2012) 023.

[24] CMS Collaboration, Search for new physics in final states with a lepton and missing transverse energy in pp collisions at the LHC, Phys. Rev. D 87, 072005 (2013).

[25] CMS Collaboration, CMS Physics Analysis Summary CMS-PAS-LUM-13-001 (2013).

[26] CMS Collaboration, The CMS experiment at the CERN LHC, JINST 3, S08004 (2008).

[27] CMS Collaboration, Energy calibration and resolution of the CMS electromagnetic calorimeter in pp collisions at $\sqrt{s}=7 \mathrm{TeV}$, JINST 8, P09009 (2013).

[28] CMS Collaboration, Search for heavy narrow dilepton resonances in pp collisions at $\sqrt{s}=7 \mathrm{TeV}$ and $\sqrt{s}=8 \mathrm{TeV}$, Phys. Lett. B 720, 63 (2013).

[29] CMS Collaboration, Performance of CMS muon reconstruction in pp collision events at $\sqrt{s}=7 \mathrm{TeV}$, JINST 7, P10002 (2012).

[30] S. Agostinelli et al. (GEANT4 Collaboration), GEANT4-a simulation toolkit, Nucl. Instrum. Methods Phys. Res., Sect. A 506, 250 (2003).

[31] J. Allison et al., Geant4 developments and applications, IEEE Trans. Nucl. Sci. 53, 270 (2006).

[32] V. M. Abazov et al. (D0), Search for $W^{\prime}$ Boson Resonances Decaying to a Top Quark and a Bottom Quark, Phys. Rev. Lett. 100, 211803 (2008).

[33] CMS Collaboration, Search for $\mathrm{W}^{\prime} \rightarrow$ tb decays in the lepton + jets final state in pp collisions at $\sqrt{s}=8 \mathrm{TeV}$, J. High Energy Phys. 05 (2014) 108.

[34] ATLAS Collaboration, Search for tb Resonances in ProtonProton Collisions at $\sqrt{s}=7 \mathrm{TeV}$ with the ATLAS Detector, Phys. Rev. Lett. 109, 081801 (2012).

[35] CMS Collaboration, Search for charge-asymmetric production of $\mathrm{W}^{\prime}$ bosons in top pair + jet events from pp collisions at $\sqrt{s}=7 \mathrm{TeV}$, Phys. Lett. B 717, 351 (2012).

[36] CMS Collaboration, Search for a $\mathrm{W}^{\prime}$ boson decaying to a bottom quark and a top quark in $\mathrm{pp}$ collisions at $\sqrt{s}=7$ TeV, Phys. Lett. B 718, 1229 (2013).

[37] ATLAS Collaboration, Search for $\mathrm{W}^{\prime} \rightarrow \mathrm{tb} \rightarrow$ qqbb Decays in pp Collisions at $\sqrt{s}=8 \mathrm{TeV}$ with the ATLAS Detector, arXiv:1408.0886.

[38] CMS Collaboration, Search for a $\mathrm{W}^{\prime}$ or Techni- $\rho$ Decaying into WZ in pp Collisions at $\sqrt{s}=7 \mathrm{TeV}$, Phys. Rev. Lett. 109, 141801 (2012).

[39] CMS Collaboration, Search for new resonances decaying via WZ to leptons in proton-proton collisions at $\sqrt{s}=8$ TeV, Phys. Lett. B 740, 83 (2014).

[40] J. C. Pati and A. Salam, Lepton number as the fourth 'color', Phys. Rev. D 10, 275 (1974).

[41] R. N. Mohapatra and J. C. Pati, 'Natural' left-right symmetry, Phys. Rev. D 11, 2558 (1975). 
[42] G. Senjanović and R. N. Mohapatra, Exact left-right symmetry and spontaneous violation of parity, Phys. Rev. D 12, 1502 (1975).

[43] G. Senjanović, Spontaneous breakdown of parity in a class of gauge theories, Nucl. Phys. B153, 334 (1979).

[44] P. Minkowski, $\mu \rightarrow \mathrm{e} \gamma$ at a rate of one out of $10^{9}$ muon decays? Phys. Lett. B 67, 421 (1977).

[45] R. N. Mohapatra and G. Senjanović, Neutrino Mass and Spontaneous Parity Nonconservation, Phys. Rev. Lett. 44, 912 (1980).

[46] R. N. Mohapatra and G. Senjanović, Neutrino masses and mixings in gauge models with spontaneous parity violation, Phys. Rev. D 23, 165 (1981).

[47] T. Sjöstrand, S. Mrenna, and P.Z. Skands, PYTHIA 6.4 physics and manual, J. High Energy Phys. 05 (2006) 026.

[48] J. Pumplin, D. R. Stump, J. Huston, H. L. Lai, P. Nadolsky, and W. K. Tung, New generation of parton distributions with uncertainties from global QCD analysis, J. High Energy Phys. 07 (2002) 012.

[49] R. Gavin, Y. Li, F. Petriello, and S. Quackenbush, FEWZ 2.0: A code for hadronic $\mathrm{Z}$ production at next-to-next-toleading order, Comput. Phys. Commun. 182, 2388 (2011).

[50] R. Gavin, Y. Li, F. Petriello, and S. Quackenbush, W Physics at the LHC with FEWZ 2.1, Comput. Phys. Commun. 184, 208 (2013).

[51] H. Terazawa, M. Yasue, K. Akama, and M. Hayashi, Observable effects of the possible substructure of leptons and quarks, Phys. Lett. B 112, 387 (1982).

[52] T. Affolder et al. (CDF Collaboration), Search for Quark Lepton Compositeness and a Heavy $W^{\prime}$ Boson Using the e $\nu$ Channel in p $\overline{\mathrm{p}}$ Collisions at $\sqrt{s}=1.8 \mathrm{TeV}$, Phys. Rev. Lett. 87, 231803 (2001).

[53] CMS Collaboration, Search for dark matter, extra dimensions, and unparticles in monojet events in proton-proton collisions at $\sqrt{s}=8 \mathrm{TeV}$, arXiv:1408.3583 [Eur. Phys. J. C (to be published)].

[54] CMS Collaboration, Search for new phenomena in monophoton final states in proton-proton collisions at $\sqrt{s}=8 \mathrm{TeV}$, arXiv:1410.8812 [Phys. Lett. B (to be published)].

[55] Yang Bai and Tim M. P. Tait, Searches with mono-leptons, Phys. Lett. B 723, 384 (2013).

[56] J. Beringer et al. (Particle Data Group), Review of particle physics, Phys. Rev. D 86, 010001 (2012).

[57] J. Alwall, M. Herquet, F. Maltoni, O. Mattelaer, and T. Stelzer, MadGraph 5: going beyond, J. High Energy Phys. 06 (2011) 128.

[58] J. Alwall, P. Demin, S. de Vischer, R. Frederix, M. Herquet, F. Maltoni, T. Plehn, D. L. Rainwater, and T. Stelzer, MadGraph/MadEvent v4: The new web generation, J. High Energy Phys. 09 (2007) 028.

[59] R. Field, 22nd Hadron Collider Physics Symposium (HCP 2010), Toronto, ON, 2010, edited by William Trischuk (TRIUMF, Vancouver, 2010).

[60] CMS Collaboration, CMS Physics Analysis Summary Report No. CMS-PAS-PFT-09-001, 2009, http://cdsweb .cern.ch/record/1194487.

[61] CMS Collaboration, CMS Physics Analysis Summary Report No. CMS-PAS-PFT-10-001, 2010, http://cdsweb .cern.ch/record/1247373.
[62] CMS Collaboration, CMS Physics Analysis Summary Report No. CMS-PAS-PFT-10-002, 2010, http://cdsweb .cern.ch/record/1279341.

[63] CMS Collaboration, Determination of jet energy calibration and transverse momentum resolution in CMS, JINST 6 , P11002 (2011).

[64] CMS Collaboration, Missing transverse energy performance of the CMS detector, JINST 6, P09001 (2011).

[65] CMS Collaboration, Measurements of Inclusive $\mathrm{W}$ and $\mathrm{Z}$ Cross Sections in pp Collisions at $\sqrt{s}=7 \mathrm{TeV}$, J. High Energy Phys. 01 (2011) 080.

[66] CMS Collaboration, CMS Detector Performance Summary Report No. CMS-DP-2013-009, 2013, http://cds.cern.ch/ record/1536406.

[67] F. Garwood, "Fiducial limits for the poisson distribution, Biometrika 28, 437 (1936).

[68] S. Frixione and B. R. Webber, Matching NLO QCD computations and parton shower simulations, J. High Energy Phys. 06 (2002) 029.

[69] S. Frixione, P. Nason, and B. R. Webber, Matching NLO QCD and parton showers in heavy flavor production, J. High Energy Phys. 08 (2003) 007.

[70] G. Corcella, I. G. Knowles, G. Marchesini, S. Moretti, K. Odagiri, P. Richardson, M. H. Seymour, and B. R. Webber, "HERWIG 6: an event generator for hadron emission reactions with interfering gluons (including supersymmetric processes), J. High Energy Phys. 01 (2001) 010.

[71] P. Nason, A new method for combining NLO QCD with shower Monte Carlo algorithms, J. High Energy Phys. 11 (2004) 040.

[72] S. Frixione, P. Nason, and C. Oleari, Matching NLO QCD computations with Parton Shower simulations: the POWHEG method, J. High Energy Phys. 11 (2007) 070.

[73] S. Alioli, P. Nason, C. Oleari, and E. Re, A general framework for implementing NLO calculations in shower Monte Carlo programs: the POWHEG BOX, J. High Energy Phys. 06 (2010) 043.

[74] E. Re, Single-top Wt-channel production matched with parton showers using the POWHEG method, Eur. Phys. J. C 71, 1547 (2011).

[75] M. Czakon, P. Fiedler, and A. Mitov, Total Top-Quark PairProduction Cross Section at Hadron Colliders Through $O\left(\alpha_{S}^{4}\right)$, Phys. Rev. Lett. 110, 252004 (2013).

[76] S. Alioli, P. Nason, C. Oleari, and E. Re, Vector boson plus one jet production in POWHEG, J. High Energy Phys. 01 (2011) 095.

[77] C. M. Carloni Calame, G. Montagna, O. Nicrosini, and A. Vicini, Precision electroweak calculation of the production of a high transverse-momentum lepton pair at hadron colliders, J. High Energy Phys. 10 (2007) 109.

[78] H.-L. Lai, M. Guzzi, J. Huston, Z. Li, P. M. Nadolsky, J. Pumplin, and C.-P. Yuan, New parton distributions for collider physics, Phys. Rev. D 82, 074024 (2010).

[79] G. Balossini, G. Montagna, C. Michel Carloni Calame, M. Moretti, O. Nicrosini, F. Piccinini, M. Treccani, and A. Vicini, Combination of electroweak and QCD corrections to single $\mathrm{W}$ production at the Fermilab Tevatron and the CERN LHC, J. High Energy Phys. 01 (2010) 013.

[80] S. Alekhin et al., The PDF4LHC Working Group Interim Report, arXiv:1101.0536. 
[81] M. Botje, J. Butterworth, A. Cooper-Sarkar, A. de Roeck, J. Feltesse, S. Forte, A. Glazov, J. Huston, R. McNulty, T. Sjöstrand, and R.S. Thorne, The PDF4LHC Working Group Interim Recommendations, arXiv:1101.0538.

[82] R. D. Ball, V. Bertone, F. Cerutti, L. Del Debbio, S. Forte, A. Guffanti, J. I. Latorre, J. Rojo, and M. Ubiali (NNPDF Collaboration), Impact of heavy quark masses on parton distributions and LHC phenomenology, Nucl. Phys. B849, 296 (2011).

[83] A. D. Martin, W. J. Stirling, R. S. Thorne, and G. Watt, Parton distributions for the LHC, Eur. Phys. J. C 63, 189 (2009).

[84] A. L. Read, Presentation of search results: The $C L_{s}$ technique, J. Phys. G 28, 2693 (2002).
[85] T. Junk, Confidence level computation for combining searches with small statistics, Nucl. Instrum. Methods Phys. Res., Sect. A 434, 435 (1999).

[86] Y. Bai, P. J. Fox, and R. Harnik, The Tevatron at the frontier of dark matter direct detection, J. High Energy Phys. 12 (2010) 048.

[87] O. Buchmueller, M. J. Dolan, and C. McCabe, Beyond effective field theory for dark matter searches at the LHC, J. High Energy Phys. 01 (2014) 025.

[88] K. -M. Cheung and G. L. Landsberg, Kaluza-Klein states of the standard model gauge bosons: constraints from high energy experiments, Phys. Rev. D 65, 076003 (2002).

V. Khachatryan, ${ }^{1}$ A. M. Sirunyan, ${ }^{1}$ A. Tumasyan, ${ }^{1}$ W. Adam, ${ }^{2}$ T. Bergauer, ${ }^{2}$ M. Dragicevic, ${ }^{2}$ J. Erö, ${ }^{2}$ C. Fabjan, ${ }^{2, b}$ M. Friedl, ${ }^{2}$ R. Frühwirth, ${ }^{2, b}$ V. M. Ghete, ${ }^{2}$ C. Hartl, ${ }^{2}$ N. Hörmann, ${ }^{2}$ J. Hrubec, ${ }^{2}$ M. Jeitler, ${ }^{2, b}$ W. Kiesenhofer, ${ }^{2}$ V. Knünz, ${ }^{2}$ M. Krammer, ${ }^{2, b}$ I. Krätschmer, ${ }^{2}$ D. Liko, ${ }^{2}$ I. Mikulec, ${ }^{2}$ D. Rabady, ${ }^{2, \mathrm{c}}$ B. Rahbaran, ${ }^{2}$ H. Rohringer, ${ }^{2}$ R. Schöfbeck, ${ }^{2}$ J. Strauss, ${ }^{2}$ A. Taurok, ${ }^{2}$ W. Treberer-Treberspurg, ${ }^{2}$ W. Waltenberger, ${ }^{2}$ C.-E. Wulz, ${ }^{2, b}$ V. Mossolov, ${ }^{3}$ N. Shumeiko, ${ }^{3}$ J. Suarez Gonzalez, ${ }^{3}$ S. Alderweireldt, ${ }^{4}$ M. Bansal, ${ }^{4}$ S. Bansal, ${ }^{4}$ T. Cornelis, ${ }^{4}$ E. A. De Wolf, ${ }^{4}$ X. Janssen, ${ }^{4}$ A. Knutsson, ${ }^{4}$ S. Luyckx, ${ }^{4}$ S. Ochesanu, ${ }^{4}$ B. Roland, ${ }^{4}$ R. Rougny, ${ }^{4}$ M. Van De Klundert, ${ }^{4}$ H. Van Haevermaet, ${ }^{4}$ P. Van Mechelen, ${ }^{4}$

N. Van Remortel, ${ }^{4}$ A. Van Spilbeeck, ${ }^{4}$ F. Blekman, ${ }^{5}$ S. Blyweert, ${ }^{5}$ J. D'Hondt, ${ }^{5}$ N. Daci, ${ }^{5}$ N. Heracleous, ${ }^{5}$

A. Kalogeropoulos, ${ }^{5}$ J. Keaveney, ${ }^{5}$ T. J. Kim, ${ }^{5}$ S. Lowette, ${ }^{5}$ M. Maes, ${ }^{5}$ A. Olbrechts, ${ }^{5}$ Q. Python, ${ }^{5}$ D. Strom, ${ }^{5}$ S. Tavernier, ${ }^{5}$ W. Van Doninck, ${ }^{5}$ P. Van Mulders, ${ }^{5}$ G. P. Van Onsem, ${ }^{5}$ I. Villella, ${ }^{5}$ C. Caillol, ${ }^{6}$ B. Clerbaux, ${ }^{6}$ G. De Lentdecker, ${ }^{6}$ D. Dobur, ${ }^{6}$ L. Favart, ${ }^{6}$ A. P. R. Gay, ${ }^{6}$ A. Grebenyuk, ${ }^{6}$ A. Léonard, ${ }^{6}$ A. Mohammadi, ${ }^{6}$ L. Perniè, ${ }^{6, c}$ T. Reis, ${ }^{6}$ T. Seva, ${ }^{6}$ L. Thomas, ${ }^{6}$ C. Vander Velde, ${ }^{6}$ P. Vanlaer, ${ }^{6}$ J. Wang, ${ }^{6}$ V. Adler, ${ }^{7}$ K. Beernaert, ${ }^{7}$ L. Benucci, ${ }^{7}$ A. Cimmino, ${ }^{7}$ S. Costantini, ${ }^{7}$ S. Crucy, ${ }^{7}$ S. Dildick, ${ }^{7}$ A. Fagot, ${ }^{7}$ G. Garcia, ${ }^{7}$ J. Mccartin, ${ }^{7}$ A. A. Ocampo Rios, ${ }^{7}$ D. Ryckbosch, ${ }^{7}$ S. Salva Diblen, ${ }^{7}$ M. Sigamani, ${ }^{7}$ N. Strobbe, ${ }^{7}$ F. Thyssen, ${ }^{7}$ M. Tytgat ${ }^{7}$ E. Yazgan, ${ }^{7}$ N. Zaganidis, ${ }^{7}$ S. Basegmez,${ }^{8}$ C. Beluffi,${ }^{8, d}$ G. Bruno, ${ }^{8}$ R. Castello, ${ }^{8}$ A. Caudron, ${ }^{8}$ L. Ceard,${ }^{8}$ G. G. Da Silveira, ${ }^{8}$ C. Delaere, ${ }^{8}$ T. du Pree, ${ }^{8}$ D. Favart, ${ }^{8}$ L. Forthomme, ${ }^{8}$ A. Giammanco, ${ }^{8, e}$ J. Hollar, ${ }^{8}$ P. Jez,${ }^{8}$ M. Komm,${ }^{8}$ V. Lemaitre ${ }^{8}$ J. Liao, ${ }^{8}$ C. Nuttens, ${ }^{8}$ D. Pagano, ${ }^{8}$ L. Perrini, ${ }^{8}$ A. Pin, ${ }^{8}$ K. Piotrzkowski, ${ }^{8}$ A. Popov, ${ }^{8, \mathrm{f}}$ L. Quertenmont, ${ }^{8}$ M. Selvaggi, ${ }^{8}$ M. Vidal Marono, ${ }^{8}$ J. M. Vizan Garcia,${ }^{8}$ N. Beliy, ${ }^{9}$ T. Caebergs, ${ }^{9}$ E. Daubie, ${ }^{9}$ G. H. Hammad, ${ }^{9}$ W. L. Aldá Júnior, ${ }^{10}$ G. A. Alves, ${ }^{10}$ M. Correa Martins Junior, ${ }^{10}$ T. Dos Reis Martins, ${ }^{10}$ M. E. Pol, ${ }^{10}$ W. Carvalho, ${ }^{11}$ J. Chinellato, ${ }^{11, g}$ A. Custódio, ${ }^{11}$ E. M. Da Costa,${ }^{11}$ D. De Jesus Damiao, ${ }^{11}$ C. De Oliveira Martins, ${ }^{11}$ S. Fonseca De Souza, ${ }^{11}$ H. Malbouisson, ${ }^{11}$ M. Malek,${ }^{11}$ D. Matos Figueiredo, ${ }^{11}$ L. Mundim, ${ }^{11}$ H. Nogima,${ }^{11}$ W. L. Prado Da Silva, ${ }^{11}$ J. Santaolalla, ${ }^{11}$ A. Santoro, ${ }^{11}$ A. Sznajder, ${ }^{11}$ E. J. Tonelli Manganote, ${ }^{11, g}$ A. Vilela Pereira, ${ }^{11}$ C. A. Bernardes, ${ }^{12 b}$ F. A. Dias, ${ }^{12 a, h}$ T. R. Fernandez Perez Tomei, ${ }^{12 a}$ E. M. Gregores, ${ }^{12 b}$ P. G. Mercadante, ${ }^{12 b}$ S. F. Novaes, ${ }^{12 a}$ Sandra S. Padula, ${ }^{12 a}$ A. Aleksandrov, ${ }^{13}$ V. Genchev, ${ }^{13, c}$ P. Iaydjiev, ${ }^{13}$ A. Marinov, ${ }^{13}$ S. Piperov, ${ }^{13}$ M. Rodozov, ${ }^{13}$ G. Sultanov, ${ }^{13}$ M. Vutova ${ }^{13}$ A. Dimitrov ${ }^{14}$ I. Glushkov, ${ }^{14}$ R. Hadjiiska, ${ }^{14}$ V. Kozhuharov, ${ }^{14}$ L. Litov, ${ }^{14}$ B. Pavlov, ${ }^{14}$ P. Petkov, ${ }^{14}$ J. G. Bian, ${ }^{15}$ G. M. Chen, ${ }^{15}$ H. S. Chen, ${ }^{15}$ M. Chen, ${ }^{15}$ R. Du,${ }^{15}$ C. H. Jiang, ${ }^{15}$ D. Liang, ${ }^{15}$ S. Liang, ${ }^{15}$ R. Plestina, ${ }^{15, \mathrm{i}} \mathrm{J}$. Tao, ${ }^{15} \mathrm{X}$. Wang, ${ }^{15} \mathrm{Z}$. Wang, ${ }^{15} \mathrm{C}$. Asawatangtrakuldee, ${ }^{16} \mathrm{Y}$. Ban, ${ }^{16} \mathrm{Y}$. Guo, ${ }^{16} \mathrm{Q}$. Li, ${ }^{16} \mathrm{~W} . \mathrm{Li},{ }^{16} \mathrm{~S}$. Liu, ${ }^{16}$ Y. Mao, ${ }^{16}$ S. J. Qian, ${ }^{16}$ D. Wang, ${ }^{16}$ L. Zhang, ${ }^{16}$ W. Zou, ${ }^{16}$ C. Avila, ${ }^{17}$ L. F. Chaparro Sierra, ${ }^{17}$ C. Florez, ${ }^{17}$ J. P. Gomez, ${ }^{17}$ B. Gomez Moreno, ${ }^{17}$ J. C. Sanabria, ${ }^{17}$ N. Godinovic,${ }^{18}$ D. Lelas, ${ }^{18}$ D. Polic, ${ }^{18}$ I. Puljak, ${ }^{18}$ Z. Antunovic, ${ }^{19}$ M. Kovac, ${ }^{19}$ V. Brigljevic, ${ }^{20}$ K. Kadija, ${ }^{20}$ J. Luetic, ${ }^{20}$ D. Mekterovic, ${ }^{20}$ L. Sudic,${ }^{20}$ A. Attikis, ${ }^{21}$ G. Mavromanolakis, ${ }^{21}$ J. Mousa, ${ }^{21}$ C. Nicolaou, ${ }^{21}$ F. Ptochos, ${ }^{21}$ P. A. Razis, ${ }^{21}$ M. Bodlak, ${ }^{22}$ M. Finger, ${ }^{22}$ M. Finger Jr., ${ }^{22, j}$ Y. Assran, ${ }^{23, k}$ S. Elgammal, ${ }^{23,1}$

M. A. Mahmoud, ${ }^{23, m}$ A. Radi ${ }^{23,1, n}$ M. Kadastik, ${ }^{24}$ M. Murumaa, ${ }^{24}$ M. Raidal,${ }^{24}$ A. Tiko, ${ }^{24}$ P. Eerola, ${ }^{25}$ G. Fedi, ${ }^{25}$ M. Voutilainen ${ }^{25}$ J. Härkönen, ${ }^{26}$ V. Karimäki, ${ }^{26}$ R. Kinnunen, ${ }^{26}$ M. J. Kortelainen, ${ }^{26}$ T. Lampén, ${ }^{26}$ K. Lassila-Perini, ${ }^{26}$ S. Lehti, ${ }^{26}$ T. Lindén, ${ }^{26}$ P. Luukka, ${ }^{26}$ T. Mäenpää, ${ }^{26}$ T. Peltola, ${ }^{26}$ E. Tuominen,${ }^{26}$ J. Tuominiemi, ${ }^{26}$ E. Tuovinen, ${ }^{26}$ L. Wendland, ${ }^{26}$ T. Tuuva, ${ }^{27}$ M. Besancon, ${ }^{28}$ F. Couderc, ${ }^{28}$ M. Dejardin, ${ }^{28}$ D. Denegri, ${ }^{28}$ B. Fabbro, ${ }^{28}$ J. L. Faure, ${ }^{28}$

C. Favaro, ${ }^{28}$ F. Ferri, ${ }^{28}$ S. Ganjour, ${ }^{28}$ A. Givernaud ${ }^{28}$ P. Gras, ${ }^{28}$ G. Hamel de Monchenault, ${ }^{28}$ P. Jarry, ${ }^{28}$ E. Locci, ${ }^{28}$ J. Malcles, ${ }^{28}$ J. Rander ${ }^{28}$ A. Rosowsky, ${ }^{28}$ M. Titov, ${ }^{28}$ S. Baffioni, ${ }^{29}$ F. Beaudette, ${ }^{29}$ P. Busson, ${ }^{29}$ C. Charlot,${ }^{29}$ T. Dahms,${ }^{29}$ M. Dalchenko, ${ }^{29}$ L. Dobrzynski, ${ }^{29}$ N. Filipovic, ${ }^{29}$ A. Florent, ${ }^{29}$ R. Granier de Cassagnac, ${ }^{29}$ L. Mastrolorenzo, ${ }^{29}$ P. Miné, ${ }^{29}$ 
C. Mironov, ${ }^{29}$ I. N. Naranjo, ${ }^{29}$ M. Nguyen, ${ }^{29}$ C. Ochando, ${ }^{29}$ P. Paganini, ${ }^{29}$ R. Salerno, ${ }^{29}$ J. B. Sauvan, ${ }^{29}$ Y. Sirois,${ }^{29}$ C. Veelken ${ }^{29}$ Y. Yilmaz, ${ }^{29}$ A. Zabi, ${ }^{29}$ J.-L. Agram, ${ }^{30,0}$ J. Andrea, ${ }^{30}$ A. Aubin, ${ }^{30}$ D. Bloch,${ }^{30}$ J.-M. Brom,${ }^{30}$ E. C. Chabert, ${ }^{30}$ C. Collard, ${ }^{30}$ E. Conte, ${ }^{30, o}$ J.-C. Fontaine, ${ }^{30,0}$ D. Gelé, ${ }^{30}$ U. Goerlach, ${ }^{30}$ C. Goetzmann, ${ }^{30}$ A.-C. Le Bihan,${ }^{30}$ P. Van Hove,${ }^{30}$ S. Gadrat, ${ }^{31}$ S. Beauceron, ${ }^{32}$ N. Beaupere, ${ }^{32}$ G. Boudoul, ${ }^{32, \mathrm{c}}$ S. Brochet,${ }^{32}$ C. A. Carrillo Montoya, ${ }^{32}$ J. Chasserat, ${ }^{32}$ R. Chierici, ${ }^{32}$ D. Contardo, ${ }^{32, c}$ P. Depasse, ${ }^{32}$ H. El Mamouni, ${ }^{32}$ J. Fan, ${ }^{32}$ J. Fay, ${ }^{32}$ S. Gascon, ${ }^{32}$ M. Gouzevitch, ${ }^{32}$ B. Ille, ${ }^{32}$ T. Kurca, ${ }^{32}$ M. Lethuillier, ${ }^{32}$ L. Mirabito, ${ }^{32}$ S. Perries, ${ }^{32}$ J. D. Ruiz Alvarez,${ }^{32}$ D. Sabes, ${ }^{32}$ L. Sgandurra, ${ }^{32}$ V. Sordini, ${ }^{32}$

M. Vander Donckt, ${ }^{32}$ P. Verdier, ${ }^{32}$ S. Viret,${ }^{32}$ H. Xiao, ${ }^{32}$ Z. Tsamalaidze, ${ }^{33, j}$ C. Autermann, ${ }^{34}$ S. Beranek,${ }^{34}$

M. Bontenackels, ${ }^{34}$ M. Edelhoff, ${ }^{34}$ L. Feld, ${ }^{34}$ O. Hindrichs,${ }^{34}$ K. Klein, ${ }^{34}$ A. Ostapchuk, ${ }^{34}$ A. Perieanu, ${ }^{34}$ F. Raupach, ${ }^{34}$ J. Sammet, ${ }^{34}$ S. Schael, ${ }^{34}$ H. Weber, ${ }^{34}$ B. Wittmer, ${ }^{34}$ V. Zhukov, ${ }^{34, f}$ M. Ata, ${ }^{35}$ E. Dietz-Laursonn, ${ }^{35}$ D. Duchardt, ${ }^{35}$ M. Erdmann, ${ }^{35}$ S. Erdweg, ${ }^{35}$ R. Fischer, ${ }^{35}$ A. Güth, ${ }^{35}$ T. Hebbeker, ${ }^{35}$ C. Heidemann, ${ }^{35}$ K. Hoepfner, ${ }^{35}$ D. Klingebiel,${ }^{35}$ S. Knutzen, ${ }^{35}$ P. Kreuzer, ${ }^{35}$ M. Merschmeyer, ${ }^{35}$ A. Meyer, ${ }^{35}$ P. Millet,${ }^{35}$ M. Olschewski, ${ }^{35}$ K. Padeken, ${ }^{35}$ P. Papacz, ${ }^{35}$ H. Reithler, ${ }^{35}$ S. A. Schmitz, ${ }^{35}$ L. Sonnenschein, ${ }^{35}$ D. Teyssier,${ }^{35}$ S. Thüer, ${ }^{35}$ M. Weber, ${ }^{35}$ V. Cherepanov, ${ }^{36}$ Y. Erdogan, ${ }^{36}$ G. Flügge, ${ }^{36}$ H. Geenen, ${ }^{36}$ M. Geisler,${ }^{36}$ W. Haj Ahmad, ${ }^{36}$ F. Hoehle, ${ }^{36}$ B. Kargoll,,${ }^{36}$ T. Kress,${ }^{36}$ Y. Kuessel, ${ }^{36}$ J. Lingemann, ${ }^{36, \mathrm{c}}$ A. Nowack, ${ }^{36}$ I. M. Nugent ${ }^{36}$ L. Perchalla, ${ }^{36}$ O. Pooth,${ }^{36}$ A. Stahl, ${ }^{36}$ I. Asin, ${ }^{37}$ N. Bartosik, ${ }^{37}$ J. Behr, ${ }^{37}$ W. Behrenhoff, ${ }^{37}$ U. Behrens, ${ }^{37}$ A. J. Bell, ${ }^{37}$ M. Bergholz,${ }^{37, p}$ A. Bethani, ${ }^{37}$ K. Borras, ${ }^{37}$ A. Burgmeier, ${ }^{37}$ A. Cakir, ${ }^{37}$

L. Calligaris, ${ }^{37}$ A. Campbell, ${ }^{37}$ S. Choudhury, ${ }^{37}$ F. Costanza, ${ }^{37}$ C. Diez Pardos,${ }^{37}$ S. Dooling, ${ }^{37}$ T. Dorland,${ }^{37}$ G. Eckerlin, ${ }^{37}$

D. Eckstein, ${ }^{37}$ T. Eichhorn, ${ }^{37}$ G. Flucke, ${ }^{37}$ J. Garay Garcia, ${ }^{37}$ A. Geiser, ${ }^{37}$ P. Gunnellini, ${ }^{37}$ J. Hauk,${ }^{37}$ G. Hellwig, ${ }^{37}$ M. Hempel,${ }^{37}$ D. Horton, ${ }^{37}$ H. Jung, ${ }^{37}$ M. Kasemann, ${ }^{37}$ P. Katsas, ${ }^{37}$ J. Kieseler, ${ }^{37}$ C. Kleinwort, ${ }^{37}$ D. Krücker,${ }^{37}$ W. Lange, ${ }^{37}$ J. Leonard, ${ }^{37}$ K. Lipka,${ }^{37}$ A. Lobanov, ${ }^{37}$ W. Lohmann, ${ }^{37, p}$ B. Lutz,${ }^{37}$ R. Mankel, ${ }^{37}$ I. Marfin, ${ }^{37}$ I.-A. Melzer-Pellmann, ${ }^{37}$ A. B. Meyer, ${ }^{37}$ J. Mnich,${ }^{37}$ A. Mussgiller, ${ }^{37}$ S. Naumann-Emme, ${ }^{37}$ A. Nayak,${ }^{37}$ O. Novgorodova, ${ }^{37}$ F. Nowak, ${ }^{37}$ E. Ntomari, ${ }^{37}$ H. Perrey, ${ }^{37}$ D. Pitzl,${ }^{37}$ R. Placakyte, ${ }^{37}$ A. Raspereza, ${ }^{37}$ P. M. Ribeiro Cipriano, ${ }^{37}$ E. Ron, ${ }^{37}$ M. Ö. Sahin, ${ }^{37}$ J. Salfeld-Nebgen, ${ }^{37}$ P. Saxena, ${ }^{37}$ R. Schmidt, ${ }^{37, p}$ T. Schoerner-Sadenius, ${ }^{37}$ M. Schröder, ${ }^{37}$ S. Spannagel, ${ }^{37}$ A. D. R. Vargas Trevino, ${ }^{37}$ R. Walsh,${ }^{37}$ C. Wissing, ${ }^{37}$ M. Aldaya Martin, ${ }^{38}$ V. Blobel, ${ }^{38}$ M. Centis Vignali, ${ }^{38}$ J. Erfle, ${ }^{38}$ E. Garutti, ${ }^{38}$ K. Goebel,${ }^{38}$ M. Görner, ${ }^{38}$ J. Haller, ${ }^{38}$ M. Hoffmann, ${ }^{38}$ R. S. Höing, ${ }^{38}$ H. Kirschenmann, ${ }^{38}$ R. Klanner,${ }^{38}$ R. Kogler, ${ }^{38}$ J. Lange, ${ }^{38}$ T. Lapsien, ${ }^{38}$ T. Lenz, ${ }^{38}$ I. Marchesini, ${ }^{38}$ J. Ott, ${ }^{38}$ T. Peiffer, ${ }^{38}$ N. Pietsch, ${ }^{38}$ D. Rathjens, ${ }^{38}$ C. Sander, ${ }^{38}$ H. Schettler, ${ }^{38}$ P. Schleper, ${ }^{38}$ E. Schlieckau, ${ }^{38}$ A. Schmidt,${ }^{38}$ M. Seidel,${ }^{38}$ J. Sibille, ${ }^{38, q}$ V. Sola, ${ }^{38}$ H. Stadie,${ }^{38}$ G. Steinbrück,${ }^{38}$ D. Troendle, ${ }^{38}$ E. Usai,${ }^{38}$ L. Vanelderen, ${ }^{38}$ C. Barth,${ }^{39}$ C. Baus, ${ }^{39}$ J. Berger,${ }^{39}$ C. Böser,${ }^{39}$ E. Butz,${ }^{39}$ T. Chwalek, ${ }^{39}$ W. De Boer, ${ }^{39}$ A. Descroix ${ }^{39}$ A. Dierlamm, ${ }^{39}$ M. Feindt, ${ }^{39}$ F. Frensch, ${ }^{39}$ M. Giffels, ${ }^{39}$ F. Hartmann, ${ }^{39, c}$ T. Hauth, ${ }^{39, \mathrm{c}}$ U. Husemann, ${ }^{39}$ I. Katkov, ${ }^{39, \mathrm{f}}$ A. Kornmayer, ${ }^{39, \mathrm{c}}$ E. Kuznetsova, ${ }^{39}$ P. Lobelle Pardo, ${ }^{39}$ M. U. Mozer, ${ }^{39}$ Th. Müller, ${ }^{39}$ A. Nürnberg, ${ }^{39}$ G. Quast, ${ }^{39}$ K. Rabbertz, ${ }^{39}$ F. Ratnikov, ${ }^{39}$ S. Röcker, ${ }^{39}$ H. J. Simonis, ${ }^{39}$ F. M. Stober, ${ }^{39}$ R. Ulrich, ${ }^{39}$ J. Wagner-Kuhr, ${ }^{39}$ S. Wayand, ${ }^{39}$ T. Weiler, ${ }^{39}$ R. Wolf, ${ }^{39}$ G. Anagnostou, ${ }^{40}$ G. Daskalakis, ${ }^{40}$ T. Geralis, ${ }^{40}$ V. A. Giakoumopoulou, ${ }^{40}$ A. Kyriakis,${ }^{40}$ D. Loukas, ${ }^{40}$ A. Markou, ${ }^{40}$ C. Markou, ${ }^{40}$ A. Psallidas, ${ }^{40}$ I. Topsis-Giotis ${ }^{40}$ A. Panagiotou, ${ }^{41}$ N. Saoulidou, ${ }^{41}$ E. Stiliaris, ${ }^{41}$ X. Aslanoglou, ${ }^{42}$ I. Evangelou ${ }^{42}$ G. Flouris, ${ }^{42}$ C. Foudas, ${ }^{42}$ P. Kokkas, ${ }^{42}$

N. Manthos, ${ }^{42}$ I. Papadopoulos, ${ }^{42}$ E. Paradas, ${ }^{42}$ G. Bencze, ${ }^{43}$ C. Hajdu, ${ }^{43}$ P. Hidas, ${ }^{43}$ D. Horvath, ${ }^{43, r}$ F. Sikler ${ }^{43}$ V. Veszpremi, ${ }^{43}$ G. Vesztergombi, ${ }^{43, \mathrm{~s}}$ A. J. Zsigmond, ${ }^{43}$ N. Beni, ${ }^{44}$ S. Czellar, ${ }^{44}$ J. Karancsi, ${ }^{44, t}$ J. Molnar, ${ }^{44}$ J. Palinkas, ${ }^{44}$ Z. Szillasi ${ }^{44}$ P. Raics,${ }^{45}$ Z. L. Trocsanyi, ${ }^{45}$ B. Ujvari, ${ }^{45}$ S. K. Swain, ${ }^{46}$ S. B. Beri,${ }^{47}$ V. Bhatnagar, ${ }^{47}$ N. Dhingra,${ }^{47}$ R. Gupta,${ }^{47}$ U. Bhawandeep, ${ }^{47}$ A. K. Kalsi, ${ }^{47}$ M. Kaur, ${ }^{47}$ M. Mittal ${ }^{47}$ N. Nishu, ${ }^{47}$ J. B. Singh,${ }^{47}$ Ashok Kumar, ${ }^{48}$ Arun Kumar, ${ }^{48}$ S. Ahuja, ${ }^{48}$ A. Bhardwaj, ${ }^{48}$ B. C. Choudhary, ${ }^{48}$ A. Kumar ${ }^{48}$ S. Malhotra, ${ }^{48}$ M. Naimuddin ${ }^{48}$ K. Ranjan, ${ }^{48}$ V. Sharma, ${ }^{48}$ S. Banerjee, ${ }^{49}$ S. Bhattacharya, ${ }^{49}$ K. Chatterjee, ${ }^{49}$ S. Dutta, ${ }^{49}$ B. Gomber, ${ }^{49}$ Sa. Jain, ${ }^{49}$ Sh. Jain, ${ }^{49}$ R. Khurana, ${ }^{49}$ A. Modak, ${ }^{49}$ S. Mukherjee, ${ }^{49}$ D. Roy, ${ }^{49}$ S. Sarkar, ${ }^{49}$ M. Sharan, ${ }^{49}$ A. Abdulsalam,${ }^{50}$ D. Dutta, ${ }^{50}$ S. Kailas,${ }^{50}$ V. Kumar, ${ }^{50}$ A. K. Mohanty, ${ }^{50, \mathrm{c}}$ L. M. Pant, ${ }^{50}$ P. Shukla, ${ }^{50}$ A. Topkar, ${ }^{50}$ T. Aziz, ${ }^{51}$ S. Banerjee, ${ }^{51}$ R. M. Chatterjee, ${ }^{51}$ R. K. Dewanjee, ${ }^{51}$ S. Dugad, ${ }^{51}$ S. Ganguly, ${ }^{51}$ S. Ghosh,${ }^{51}$ M. Guchait, ${ }^{51}$ A. Gurtu, ${ }^{51, u}$ G. Kole, ${ }^{51}$ S. Kumar, ${ }^{51}$ M. Maity, ${ }^{51, v}$ G. Majumder,${ }^{51}$ K. Mazumdar, ${ }^{51}$ G. B. Mohanty, ${ }^{51}$ B. Parida,${ }^{51}$ K. Sudhakar, ${ }^{51}$ N. Wickramage,${ }^{51, w}$ H. Bakhshiansohi, ${ }^{52}$ H. Behnamian, ${ }^{52}$ S. M. Etesami, ${ }^{52, x}$ A. Fahim, ${ }^{52, y}$ R. Goldouzian, ${ }^{52}$ A. Jafari, ${ }^{52}$ M. Khakzad, ${ }^{52}$ M. Mohammadi Najafabadi, ${ }^{52}$ M. Naseri, ${ }^{52}$ S. Paktinat Mehdiabadi, ${ }^{52}$ B. Safarzadeh,${ }^{52, z}$ M. Zeinali, ${ }^{52}$ M. Felcini, ${ }^{53}$ M. Grunewald, ${ }^{53}$ M. Abbrescia, ${ }^{54 a, 54 b}$ L. Barbone, ${ }^{54 a, 54 b}$ C. Calabria, ${ }^{54,54 b}$ S. S. Chhibra, ${ }^{54,54 b}$ A. Colaleo, ${ }^{54 a}$ D. Creanza, ${ }^{54 a, 54 c}$ N. De Filippis, ${ }^{54 a, 54 c}$ M. De Palma ${ }^{54 a, 54 b}$ L. Fiore,${ }^{54 a}$ G. Iaselli, ${ }^{54,54 c}$ G. Maggi, ${ }^{54 a, 54 c}$ M. Maggi, ${ }^{54 a}$ S. My,${ }^{54 a, 54 c}$ S. Nuzzo,${ }^{54 a, 54 b}$ A. Pompili, ${ }^{54 a, 54 b}$ G. Pugliese,${ }^{54,54 \mathrm{c}}$ R. Radogna, ${ }^{54 a, 54 b, c}$ G. Selvaggi, ${ }^{54 a, 54 b}$ L. Silvestris, ${ }^{54 a, c}$ G. Singh ${ }^{54 a, 54 b}$ R. Venditti, ${ }^{54,54 b}$ P. Verwilligen, ${ }^{54 a}$ G. Zito, ${ }^{54 \mathrm{a}}$ G. Abbiendi, ${ }^{55 \mathrm{a}}$ A. C. Benvenuti, ${ }^{55 \mathrm{a}}$ D. Bonacorsi, ${ }^{55 \mathrm{a}, 55 \mathrm{~b}}$ S. Braibant-Giacomelli, ${ }^{55,55 \mathrm{~b}}$ L. Brigliadori, ${ }^{55 \mathrm{a}, 55 \mathrm{~b}}$ 
R. Campanini ${ }^{55 a, 55 b}$ P. Capiluppi ${ }^{55 a, 55 b}$ A. Castro, ${ }^{55 a, 55 b}$ F. R. Cavallo, ${ }^{55 a}$ G. Codispoti, ${ }^{55 a, 55 b}$ M. Cuffiani, ${ }^{55 a, 55 b}$ G. M. Dallavalle, ${ }^{55 a}$ F. Fabbri, ${ }^{55 a}$ A. Fanfani, ${ }^{55 a, 55 b}$ D. Fasanella, ${ }^{55 a, 55 b}$ P. Giacomelli, ${ }^{55 a}$ C. Grandi, ${ }^{55 a}$ L. Guiducci, ${ }^{55 a, 55 b}$ S. Marcellini, ${ }^{55 a}$ G. Masetti, ${ }^{55, c}$ A. Montanari, ${ }^{55 a}$ F. L. Navarria, ${ }^{55 a, 55 b}$ A. Perrotta, ${ }^{55 a}$ F. Primavera ${ }^{55 a, 55 b}$ A. M. Rossi ${ }^{55 a, 55 b}$ T. Rovelli, ${ }^{55 a, 55 b}$ G. P. Siroli, ${ }^{55 a, 55 b}$ N. Tosi, ${ }^{55 a, 55 b}$ R. Travaglini, ${ }^{55 a, 55 b}$ S. Albergo, ${ }^{56 a, 56 b}$ G. Cappello, ${ }^{56 a}$ M. Chiorboli, ${ }^{56 a, 56 b}$ S. Costa, ${ }^{56 a, 56 b}$ F. Giordano, ${ }^{56 a, 56 c, c}$ R. Potenza, ${ }^{56 a, 56 b}$ A. Tricomi, ${ }^{56 a, 56 b}$ C. Tuve, ${ }^{56 a, 56 b}$ G. Barbagli, ${ }^{57 a}$ V. Ciulli, ${ }^{57 a, 57 b}$ C. Civinini, ${ }^{57 a}$ R. D’Alessandro, ${ }^{57 a, 57 b}$ E. Focardi, ${ }^{57 a, 57 b}$ E. Gallo, ${ }^{57 a}$ S. Gonzi, ${ }^{57 a, 57 b}$ V. Gori, ${ }^{57 a, 57 b, c}$ P. Lenzi, ${ }^{57 a, 57 b}$ M. Meschini ${ }^{57 \mathrm{a}}$ S. Paoletti ${ }^{57 \mathrm{a}}$ G. Sguazzoni ${ }^{57 \mathrm{a}}$ A. Tropiano, ${ }^{57 \mathrm{a}, 57 \mathrm{~b}}$ L. Benussi,${ }^{58}$ S. Bianco,${ }^{58}$ F. Fabbri,${ }^{58}$ D. Piccolo,${ }^{58}$ F. Ferro, ${ }^{59 a}$ M. Lo Vetere, ${ }^{59 a, 59 b}$ E. Robutti, ${ }^{59 a}$ S. Tosi, ${ }^{59 a, 59 b}$ M. E. Dinardo, ${ }^{60 a, 60 b}$ S. Fiorendi, ${ }^{60 a, 60 b, c}$ S. Gennai, ${ }^{60 a, c}$ R. Gerosa, ${ }^{60 a, c}$ A. Ghezzi, ${ }^{60 a, 60 b}$ P. Govoni, ${ }^{60 a, 60 b}$ M. T. Lucchini, ${ }^{60 a, 60 b, c}$ S. Malvezzi, ${ }^{60 a}$ R. A. Manzoni, ${ }^{60 a, 60 b}$ A. Martelli, ${ }^{60 \mathrm{a}, 60 \mathrm{~b}}$ B. Marzocchi, ${ }^{60 \mathrm{a}}$ D. Menasce ${ }^{60 \mathrm{a}}$ L. Moroni, ${ }^{60 \mathrm{a}}$ M. Paganoni, ${ }^{60,60 \mathrm{~b}}$ D. Pedrini, ${ }^{60 \mathrm{a}}$ S. Ragazzi, ${ }^{60,60 \mathrm{~b}}$ N. Redaelli, ${ }^{60 \mathrm{a}}$ T. Tabarelli de Fatis, ${ }^{60 a, 60 \mathrm{~b}}$ S. Buontempo, ${ }^{61 \mathrm{a}}$ N. Cavallo, ${ }^{61 \mathrm{a}, 61 \mathrm{c}} \mathrm{S}$. Di Guida, ${ }^{61 \mathrm{a}, 61 \mathrm{~d}, \mathrm{c}}$ F. Fabozzi, ${ }^{61 \mathrm{a}, 61 \mathrm{c}}$ A. O. M. Iorio, ${ }^{61 a, 61 b}$ L. Lista, ${ }^{61 a}$ S. Meola ${ }^{61 a, 61 d, c}$ M. Merola,${ }^{61 a}$ P. Paolucci, ${ }^{61 a, c}$ P. Azzi, ${ }^{62 a}$ N. Bacchetta, ${ }^{62 a}$ D. Bisello, ${ }^{62 a, 62 b}$ A. Branca, ${ }^{62 a, 62 b}$ R. Carlin, ${ }^{62 a, 62 b}$ P. Checchia, ${ }^{62 a}$ M. Dall'Osso, ${ }^{62 a, 62 b}$ T. Dorigo, ${ }^{62 a}$ S. Fantinel, ${ }^{62 a}$ M. Galanti, ${ }^{62 a, 62 b}$ F. Gasparini, ${ }^{62 a, 62 b}$ U. Gasparini, ${ }^{62 a, 62 b}$ P. Giubilato, ${ }^{62 a, 62 b}$ A. Gozzelino, ${ }^{62 a}$ K. Kanishchev, ${ }^{62 a, 62 c}$ S. Lacaprara, ${ }^{62 a}$ M. Margoni, ${ }^{62 a, 62 b}$ A. T. Meneguzzo, ${ }^{62 a, 62 b}$ J. Pazzini, ${ }^{62 a, 62 b}$ N. Pozzobon, ${ }^{62 a, 62 b}$ P. Ronchese, ${ }^{62 a, 62 b}$ F. Simonetto, ${ }^{62 a, 62 b}$ E. Torassa, ${ }^{62 a}$ M. Tosi, ${ }^{62 a, 62 b}$ P. Zotto, ${ }^{62 a, 62 b}$ A. Zucchetta, ${ }^{62 a, 62 b}$ G. Zumerle, ${ }^{62 a, 62 b}$ M. Gabusi, ${ }^{63 a, 63 b}$ S. P. Ratti, ${ }^{6 a, 63 b}$ C. Riccardi, ${ }^{63 a, 63 b}$ P. Salvini, ${ }^{63 a}$ P. Vitulo, ${ }^{63 a, 63 b}$ M. Biasini ${ }^{64 a, 64 b}$ G. M. Bilei, ${ }^{64 a}$ D. Ciangottini, ${ }^{64 a, 64 b}$ L. Fanò, ${ }^{64 a, 64 b}$ P. Lariccia,${ }^{64 a, 64 b}$ G. Mantovani, ${ }^{64 a, 64 b}$ M. Menichelli, ${ }^{64 a}$ F. Romeo, ${ }^{64 a, 64 b}$ A. Saha, ${ }^{64 a}$ A. Santocchia,${ }^{64 a, 64 b}$ A. Spiezia, ${ }^{64 a, 64 b, c}$ K. Androsov, ${ }^{65 a, a a}$ P. Azzurri, ${ }^{65 a}$ G. Bagliesi, ${ }^{65 a}$ J. Bernardini, ${ }^{65 a}$ T. Boccali, ${ }^{65 a}$ G. Broccolo,${ }^{65 a, 65 c}$ R. Castaldi, ${ }^{65 a}$ M. A. Ciocci, ${ }^{65 a, a a}$ R. Dell'Orso, ${ }^{65 a}$ S. Donato, ${ }^{65,65 c}$ F. Fiori, ${ }^{65 a, 65 c}$ L. Foà, ${ }^{65 a, 65 c}$ A. Giassi, ${ }^{65 a}$ M. T. Grippo, ${ }^{65 a, a a}$ F. Ligabue ${ }^{65 a, 65 c}$ T. Lomtadze, ${ }^{65 a}$ L. Martini, ${ }^{65 a, 65 b}$ A. Messineo,${ }^{65 a, 65 b}$ C. S. Moon, ${ }^{65 a, b b}$ F. Palla, ${ }^{65 a, c}$ A. Rizzi, ${ }^{65 a, 65 b}$ A. Savoy-Navarro, ${ }^{65 a, c c}$ A. T. Serban, ${ }^{65 a}$ P. Spagnolo, ${ }^{65 a}$ P. Squillacioti, ${ }^{65 a, a a}$ R. Tenchini, ${ }^{65 a}$ G. Tonelli,${ }^{65 a, 65 b}$ A. Venturi, ${ }^{65 a}$ P. G. Verdini, ${ }^{65 \mathrm{a}}$ C. Vernieri, ${ }^{65 \mathrm{a}, 65 \mathrm{c}, \mathrm{c}}$ L. Barone,${ }^{66 \mathrm{a}, 66 \mathrm{~b}}$ F. Cavallari, ${ }^{66 \mathrm{a}}$ D. Del Re,${ }^{66 \mathrm{a}, 66 \mathrm{~b}}$ M. Diemoz ${ }^{66 \mathrm{a}}$ M. Grassi, ${ }^{66 a, 66 \mathrm{~b}}$ C. Jorda, ${ }^{66 \mathrm{a}}$ E. Longo, ${ }^{66 \mathrm{a}, 66 \mathrm{~b}}$ F. Margaroli, ${ }^{66 \mathrm{a}, 66 \mathrm{~b}}$ P. Meridiani, ${ }^{66 \mathrm{a}}$ F. Micheli, ${ }^{66 a, 66 \mathrm{~b}, \mathrm{c}}$ S. Nourbakhsh, ${ }^{66 \mathrm{a}, 66 \mathrm{~b}}$ G. Organtini, ${ }^{66 \mathrm{a}, 66 \mathrm{~b}}$ R. Paramatti, ${ }^{66 \mathrm{a}}$ S. Rahatlou, ${ }^{66 \mathrm{a}, 66 \mathrm{~b}}$ C. Rovelli, ${ }^{66 \mathrm{a}}$ F. Santanastasio, ${ }^{66 \mathrm{a}, 66 \mathrm{~b}}$ L. Soffi, ${ }^{66 a, 66 \mathrm{~b}, \mathrm{c}}$ P. Traczyk, ${ }^{66 \mathrm{a}, 66 \mathrm{~b}}$ N. Amapane, ${ }^{67 \mathrm{a}, 67 \mathrm{~b}}$ R. Arcidiacono, ${ }^{67 a, 67 c}$ S. Argiro, ${ }^{67 a, 67 b, c}$ M. Arneodo, ${ }^{67 a, 67 c}$ R. Bellan, ${ }^{67 a, 67 b}$ C. Biino, ${ }^{67 a}$ N. Cartiglia, ${ }^{67 a}$ S. Casasso, ${ }^{67 a, 67 b, c}$ M. Costa, ${ }^{67 a, 67 b}$ A. Degano, ${ }^{67 a, 67 b}$ N. Demaria,${ }^{67 a}$ L. Finco, ${ }^{67 a, 67 b}$ C. Mariotti ${ }^{67 a}$ S. Maselli, ${ }^{67 a}$ E. Migliore,${ }^{67 a, 67 b}$ V. Monaco, ${ }^{67 a, 67 b}$ M. Musich, ${ }^{67 a}$ M. M. Obertino ${ }^{67 a, 67 c, c}$ G. Ortona,${ }^{67 a, 67 b}$ L. Pacher, ${ }^{67 a, 67 b}$ N. Pastrone, ${ }^{67 a}$ M. Pelliccioni, ${ }^{67 a}$ G. L. Pinna Angioni, ${ }^{67 a, 67 b}$ A. Potenza, ${ }^{67 a, 67 b}$ A. Romero, ${ }^{67 a, 67 b}$ M. Ruspa,${ }^{67 a, 67 c}$ R. Sacchi, ${ }^{67 a, 67 b}$ A. Solano, ${ }^{67 a, 67 b}$ A. Staiano, ${ }^{67 \mathrm{a}}$ U. Tamponi, ${ }^{67 \mathrm{a}}$ S. Belforte, ${ }^{68 \mathrm{a}}$ V. Candelise, ${ }^{68 \mathrm{a}, 68 \mathrm{~b}}$ M. Casarsa,${ }^{68 \mathrm{a}}$ F. Cossutti, ${ }^{68 \mathrm{a}}$ G. Della Ricca, ${ }^{68 \mathrm{a}, 68 \mathrm{~b}}$ B. Gobbo, ${ }^{68 \mathrm{a}}$ C. La Licata, ${ }^{68 \mathrm{a}, 68 \mathrm{~b}}$ M. Marone ${ }^{68 \mathrm{a}, 68 \mathrm{~b}}$ D. Montanino, ${ }^{68 \mathrm{a}, 68 \mathrm{~b}}$ A. Schizzi, ${ }^{68 \mathrm{a}, 68 \mathrm{~b}, \mathrm{c}}$ T. Umer, ${ }^{68 \mathrm{a}, 68 \mathrm{~b}}$ A. Zanetti, ${ }^{68 \mathrm{a}}$ S. Chang, ${ }^{69}$ A. Kropivnitskaya, ${ }^{69}$ S. K. Nam, ${ }^{69}$ D. H. Kim, ${ }^{70}$ G. N. Kim,${ }^{70}$ M. S. Kim, ${ }^{70}$ D. J. Kong,${ }^{70}$ S. Lee,${ }^{70}$ Y. D. Oh, ${ }^{70}$ H. Park ${ }^{70}$ A. Sakharov, ${ }^{70}$ D. C. Son,${ }^{70}$ J. Y. Kim, ${ }^{71}$ S. Song,${ }^{71}$ S. Choi, ${ }^{72}$ D. Gyun, ${ }^{72}$ B. Hong,${ }^{72}$ M. Jo, ${ }^{72}$ H. Kim, ${ }^{72}$ Y. Kim,${ }^{72}$ B. Lee, ${ }^{72}$ K. S. Lee, ${ }^{72}$ S. K. Park, ${ }^{72}$ Y. Roh,${ }^{72}$ M. Choi, ${ }^{73}$ J. H. Kim,${ }^{73}$ I. C. Park,${ }^{73}$ S. Park,${ }^{73}$ G. Ryu,${ }^{73}$ M. S. Ryu, ${ }^{73}$ Y. Choi,${ }^{74}$ Y. K. Choi,${ }^{74}$ J. Goh,${ }^{74}$ E. Kwon, ${ }^{74}$ J. Lee,${ }^{74}$ H. Seo, ${ }^{74}$ I. Yu, ${ }^{74}$ A. Juodagalvis, ${ }^{75}$ J. R. Komaragiri, ${ }^{76}$ H. Castilla-Valdez,${ }^{77}$ E. De La Cruz-Burelo, ${ }^{77}$ I. Heredia-de La Cruz, ${ }^{77, d d}$ R. Lopez-Fernandez ${ }^{77}$ A. Sanchez-Hernandez, ${ }^{77}$ S. Carrillo Moreno, ${ }^{78}$ F. Vazquez Valencia ${ }^{78}$ I. Pedraza,${ }^{79}$ H. A. Salazar Ibarguen, ${ }^{79}$ E. Casimiro Linares, ${ }^{80}$ A. Morelos Pineda,${ }^{80}$ D. Krofcheck,${ }^{81}$ P. H. Butler, ${ }^{82}$ S. Reucroft, ${ }^{82}$ A. Ahmad ${ }^{83}$ M. Ahmad ${ }^{83}$ Q. Hassan, ${ }^{83}$ H. R. Hoorani, ${ }^{83}$ S. Khalid ${ }^{83}$ W. A. Khan, ${ }^{83}$ T. Khurshid, ${ }^{83}$ M. A. Shah,${ }^{83}$ M. Shoaib ${ }^{83}$ H. Bialkowska, ${ }^{84}$ M. Bluj, ${ }^{84}$ B. Boimska, ${ }^{84}$ T. Frueboes, ${ }^{84}$ M. Górski, ${ }^{84}$ M. Kazana ${ }^{84}$ K. Nawrocki, ${ }^{84}$ K. Romanowska-Rybinska ${ }^{84}$ M. Szleper ${ }^{84}$ P. Zalewski, ${ }^{84}$ G. Brona, ${ }^{85}$ K. Bunkowski, ${ }^{85}$ M. Cwiok, ${ }^{85}$ W. Dominik, ${ }^{85}$ K. Doroba, ${ }^{85}$ A. Kalinowski, ${ }^{85}$ M. Konecki, ${ }^{85}$ J. Krolikowski, ${ }^{85}$ M. Misiura, ${ }^{85}$ M. Olszewski, ${ }^{85}$ W. Wolszczak, ${ }^{85}$ P. Bargassa, ${ }^{86}$ C. Beirão Da Cruz E Silva,${ }^{86}$ P. Faccioli, ${ }^{86}$ P. G. Ferreira Parracho,${ }^{86}$ M. Gallinaro, ${ }^{86}$ F. Nguyen, ${ }^{86}$ J. Rodrigues Antunes, ${ }^{86}$ J. Seixas, ${ }^{86}$ J. Varela ${ }^{86}$ P. Vischia, ${ }^{86}$ P. Bunin,${ }^{87}$ M. Gavrilenko, ${ }^{87}$ I. Golutvin, ${ }^{87}$ A. Kamenev, ${ }^{87}$ V. Karjavin, ${ }^{87}$ V. Konoplyanikov, ${ }^{87}$ A. Lanev, ${ }^{87}$ A. Malakhov ${ }^{87}$ V. Matveev, ${ }^{87, e e}$ P. Moisenz, ${ }^{87}$ V. Palichik,${ }^{87}$ V. Perelygin, ${ }^{87}$ M. Savina, ${ }^{87}$ S. Shmatov, ${ }^{87}$ S. Shulha, ${ }^{87}$ N. Skatchkov, ${ }^{87}$ V. Smirnov, ${ }^{87}$ A. Zarubin, ${ }^{87}$ V. Golovtsov, ${ }^{88}$ Y. Ivanov, ${ }^{88}$ V. Kim, ${ }^{88, f f}$ P. Levchenko, ${ }^{88}$ V. Murzin,${ }^{88}$ V. Oreshkin, ${ }^{88}$ I. Smirnov, ${ }^{88}$ V. Sulimov, ${ }^{88}$ L. Uvarov,${ }^{88}$ S. Vavilov, ${ }^{88}$ A. Vorobyev, ${ }^{88}$ An. Vorobyev, ${ }^{88}$ Yu. Andreev, ${ }^{89}$ A. Dermenev, ${ }^{89}$ S. Gninenko,${ }^{89}$ N. Golubev, ${ }^{89}$ M. Kirsanov, ${ }^{89}$

N. Krasnikov, ${ }^{89}$ A. Pashenkov, ${ }^{89}$ D. Tlisov, ${ }^{89}$ A. Toropin, ${ }^{89}$ V. Epshteyn, ${ }^{90}$ V. Gavrilov, ${ }^{90}$ N. Lychkovskaya, ${ }^{90}$ V. Popov, ${ }^{90}$ G. Safronov, ${ }^{90}$ S. Semenov, ${ }^{90}$ A. Spiridonov, ${ }^{90}$ V. Stolin, ${ }^{90}$ E. Vlasov, ${ }^{90}$ A. Zhokin, ${ }^{90}$ V. Andreev, ${ }^{91}$ M. Azarkin, ${ }^{91}$ 
I. Dremin, ${ }^{91}$ M. Kirakosyan, ${ }^{91}$ A. Leonidov, ${ }^{91}$ G. Mesyats, ${ }^{91}$ S. V. Rusakov, ${ }^{91}$ A. Vinogradov, ${ }^{91}$ A. Belyaev, ${ }^{92}$ E. Boos,${ }^{92}$ V. Bunichev, ${ }^{92}$ M. Dubinin, ${ }^{92, \mathrm{~h}}$ L. Dudko, ${ }^{92}$ A. Ershov, ${ }^{92}$ A. Gribushin, ${ }^{92}$ V. Klyukhin, ${ }^{92}$ O. Kodolova, ${ }^{92}$ I. Lokhtin, ${ }^{92}$ S. Obraztsov, ${ }^{92}$ M. Perfilov, ${ }^{92}$ V. Savrin, ${ }^{92}$ I. Azhgirey, ${ }^{93}$ I. Bayshev,${ }^{93}$ S. Bitioukov, ${ }^{93}$ V. Kachanov, ${ }^{93}$ A. Kalinin, ${ }^{93}$ D. Konstantinov, ${ }^{93}$ V. Krychkine, ${ }^{93}$ V. Petrov, ${ }^{93}$ R. Ryutin, ${ }^{93}$ A. Sobol, ${ }^{93}$ L. Tourtchanovitch, ${ }^{93}$ S. Troshin,${ }^{93}$ N. Tyurin, ${ }^{93}$ A. Uzunian, ${ }^{93}$ A. Volkov, ${ }^{93}$ P. Adzic, ${ }^{94, g g}$ M. Dordevic, ${ }^{94}$ M. Ekmedzic, ${ }^{94}$ J. Milosevic, ${ }^{94}$ J. Alcaraz Maestre, ${ }^{95}$ C. Battilana,${ }^{95}$ E. Calvo, ${ }^{95}$ M. Cerrada,${ }^{95}$ M. Chamizo Llatas, ${ }^{95, c}$ N. Colino,${ }^{95}$ B. De La Cruz,${ }^{95}$ A. Delgado Peris, ${ }^{95}$ D. Domínguez Vázquez, ${ }^{95}$ A. Escalante Del Valle, ${ }^{95}$ C. Fernandez Bedoya, ${ }^{95}$ J. P. Fernández Ramos, ${ }^{95}$ J. Flix,${ }^{95}$ M. C. Fouz, ${ }^{95}$ P. Garcia-Abia, ${ }^{95}$ O. Gonzalez Lopez, ${ }^{95}$ S. Goy Lopez, ${ }^{95}$ J. M. Hernandez, ${ }^{95}$ M. I. Josa ${ }^{95}$ G. Merino, ${ }^{95}$ E. Navarro De Martino, ${ }^{95}$ A. Pérez-Calero Yzquierdo, ${ }^{95}$ J. Puerta Pelayo, ${ }^{95}$ A. Quintario Olmeda, ${ }^{95}$ I. Redondo, ${ }^{95}$ L. Romero, ${ }^{95}$ M. S. Soares, ${ }^{95}$ C. Albajar, ${ }^{96}$ J. F. de Trocóniz, ${ }^{96}$ M. Missiroli, ${ }^{96}$ H. Brun, ${ }^{97}$ J. Cuevas, ${ }^{97}$

J. Fernandez Menendez, ${ }^{97}$ S. Folgueras, ${ }^{97}$ I. Gonzalez Caballero, ${ }^{97}$ L. Lloret Iglesias, ${ }^{97}$ J. A. Brochero Cifuentes, ${ }^{98}$ I. J. Cabrillo, ${ }^{98}$ A. Calderon, ${ }^{98}$ J. Duarte Campderros, ${ }^{98}$ M. Fernandez, ${ }^{98}$ G. Gomez,${ }^{98}$ A. Graziano, ${ }^{98}$ A. Lopez Virto, ${ }^{98}$ J. Marco, ${ }^{98}$ R. Marco, ${ }^{98}$ C. Martinez Rivero, ${ }^{98}$ F. Matorras, ${ }^{98}$ F. J. Munoz Sanchez, ${ }^{98}$ J. Piedra Gomez, ${ }^{98}$ T. Rodrigo, ${ }^{98}$ A. Y. Rodríguez-Marrero, ${ }^{98}$ A. Ruiz-Jimeno,${ }^{98}$ L. Scodellaro, ${ }^{98}$ I. Vila, ${ }^{98}$ R. Vilar Cortabitarte, ${ }^{98}$ D. Abbaneo, ${ }^{99}$ E. Auffray, ${ }^{99}$ G. Auzinger, ${ }^{99}$ M. Bachtis, ${ }^{99}$ P. Baillon, ${ }^{99}$ A. H. Ball, ${ }^{99}$ D. Barney, ${ }^{99}$ A. Benaglia, ${ }^{99}$ J. Bendavid,${ }^{99}$ L. Benhabib,${ }^{99}$ J. F. Benitez, ${ }^{99}$ C. Bernet, ${ }^{99, i}$ G. Bianchi, ${ }^{99}$ P. Bloch, ${ }^{99}$ A. Bocci, ${ }^{99}$ A. Bonato,${ }^{99}$ O. Bondu, ${ }^{99}$ C. Botta, ${ }^{99}$ H. Breuker, ${ }^{99}$ T. Camporesi, ${ }^{99}$ G. Cerminara, ${ }^{99}$ S. Colafranceschi,${ }^{99, \text { hh }}$ M. D'Alfonso, ${ }^{99}$ D. d'Enterria, ${ }^{99}$ A. Dabrowski, ${ }^{99}$ A. David,${ }^{99}$ F. De Guio, ${ }^{99}$ A. De Roeck, ${ }^{99}$ S. De Visscher, ${ }^{99}$ M. Dobson, ${ }^{99}$ N. Dupont-Sagorin, ${ }^{99}$ A. Elliott-Peisert, ${ }^{99}$ J. Eugster, ${ }^{99}$ G. Franzoni, ${ }^{99}$ W. Funk,${ }^{99}$ D. Gigi,${ }^{99}$ K. Gill, ${ }^{99}$ D. Giordano, ${ }^{99}$ M. Girone,${ }^{99}$ F. Glege, ${ }^{99}$ R. Guida,${ }^{99}$ S. Gundacker, ${ }^{99}$ M. Guthoff, ${ }^{99}$ J. Hammer, ${ }^{99}$ M. Hansen,${ }^{99}$ P. Harris, ${ }^{99}$ J. Hegeman, ${ }^{99}$ V. Innocente, ${ }^{99}$ P. Janot, ${ }^{99}$ K. Kousouris, ${ }^{99}$

K. Krajczar, ${ }^{99}$ P. Lecoq ${ }^{99}$ C. Lourenço, ${ }^{99}$ N. Magini, ${ }^{99}$ L. Malgeri, ${ }^{99}$ M. Mannelli, ${ }^{99}$ J. Marrouche, ${ }^{99}$ L. Masetti, ${ }^{99}$ F. Meijers ${ }^{99}$ S. Mersi,${ }^{99}$ E. Meschi,${ }^{99}$ F. Moortgat,${ }^{99}$ S. Morovic, ${ }^{99}$ M. Mulders,${ }^{99}$ P. Musella,${ }^{99}$ L. Orsini, ${ }^{99}$ L. Pape, ${ }^{99}$ E. Perez,${ }^{99}$ L. Perrozzi,${ }^{99}$ A. Petrilli, ${ }^{99}$ G. Petrucciani, ${ }^{99}$ A. Pfeiffer,${ }^{99}$ M. Pierini, ${ }^{99}$ M. Pimiä, ${ }^{99}$ D. Piparo, ${ }^{99}$ M. Plagge,${ }^{99}$ A. Racz, ${ }^{99}$ G. Rolandi, ${ }^{99, i i}$ M. Rovere,${ }^{99}$ H. Sakulin, ${ }^{99}$ C. Schäfer, ${ }^{99}$ C. Schwick,${ }^{99}$ S. Sekmen, ${ }^{99}$ A. Sharma,${ }^{99}$ P. Siegrist,${ }^{99}$ P. Silva, ${ }^{99}$ M. Simon, ${ }^{99}$ P. Sphicas, ${ }^{99, j j}$ D. Spiga, ${ }^{99}$ J. Steggemann, ${ }^{99}$ B. Stieger,${ }^{99}$ M. Stoye, ${ }^{99}$ D. Treille, ${ }^{99}$ A. Tsirou, ${ }^{99}$ G. I. Veres, ${ }^{99, \mathrm{~s}}$ J. R. Vlimant, ${ }^{99}$ N. Wardle, ${ }^{99}$ H. K. Wöhri, ${ }^{99}$ W. D. Zeuner, ${ }^{99}$ W. Bertl, ${ }^{100}$ K. Deiters,${ }^{100}$ W. Erdmann, ${ }^{100}$ R. Horisberger, ${ }^{100}$ Q. Ingram, ${ }^{100}$ H. C. Kaestli, ${ }^{100}$ S. König, ${ }^{100}$ D. Kotlinski, ${ }^{100}$ U. Langenegger, ${ }^{100}$ D. Renker ${ }^{100}$ T. Rohe, ${ }^{100}$ F. Bachmair, ${ }^{101}$ L. Bäni, ${ }^{101}$ L. Bianchini,${ }^{101}$ P. Bortignon, ${ }^{101}$ M. A. Buchmann, ${ }^{101}$ B. Casal, ${ }^{101}$ N. Chanon,${ }^{101}$ A. Deisher, ${ }^{101}$ G. Dissertori, ${ }^{101}$ M. Dittmar, ${ }^{101}$ M. Donegà, ${ }^{101}$ M. Dünser, ${ }^{101}$ P. Eller, ${ }^{101}$ C. Grab, ${ }^{101}$ D. Hits, ${ }^{101}$ W. Lustermann, ${ }^{101}$ B. Mangano, ${ }^{101}$ A. C. Marini, ${ }^{101}$ P. Martinez Ruiz del Arbol, ${ }^{101}$ D. Meister, ${ }^{101}$ N. Mohr, ${ }^{101}$ C. Nägeli, ${ }^{101, k k}$ P. Nef, ${ }^{101}$ F. Nessi-Tedaldi, ${ }^{101}$ F. Pandolfi, ${ }^{101}$ F. Pauss, ${ }^{101}$ M. Peruzzi, ${ }^{101}$ M. Quittnat, ${ }^{101}$ L. Rebane, ${ }^{101}$ M. Rossini, ${ }^{101}$ A. Starodumov, ${ }^{101,11}$ M. Takahashi, ${ }^{101}$ K. Theofilatos, ${ }^{101}$ R. Wallny, ${ }^{101}$ H. A. Weber, ${ }^{101}$ C. Amsler, ${ }^{102, m m}$ M. F. Canelli, ${ }^{102}$ V. Chiochia, ${ }^{102}$ A. De Cosa, ${ }^{102}$ A. Hinzmann, ${ }^{102}$ T. Hreus, ${ }^{102}$ B. Kilminster, ${ }^{102}$ B. Millan Mejias, ${ }^{102}$ J. Ngadiuba, ${ }^{102}$ P. Robmann, ${ }^{102}$ F. J. Ronga, ${ }^{102}$ H. Snoek, ${ }^{102}$ S. Taroni, ${ }^{102}$ M. Verzetti, ${ }^{102}$ Y. Yang, ${ }^{102}$ M. Cardaci, ${ }^{103}$ K. H. Chen, ${ }^{103}$ C. Ferro, ${ }^{103}$ C. M. Kuo, ${ }^{103}$ W. Lin, ${ }^{103}$ Y. J. Lu, ${ }^{103}$ R. Volpe, ${ }^{103}$ S. S. Yu ${ }^{103}$ P. Chang, ${ }^{104}$ Y. H. Chang, ${ }^{104}$ Y. W. Chang, ${ }^{104}$ Y. Chao, ${ }^{104}$ K. F. Chen, ${ }^{104}$ P. H. Chen,${ }^{104}$ C. Dietz, ${ }^{104}$ U. Grundler, ${ }^{104}$ W.-S. Hou, ${ }^{104}$ K. Y. Kao, ${ }^{104}$ Y. J. Lei, ${ }^{104}$ Y. F. Liu, ${ }^{104}$ R.-S. Lu, ${ }^{104}$ D. Majumder, ${ }^{104}$ E. Petrakou, ${ }^{104}$ Y. M. Tzeng, ${ }^{104}$ R. Wilken, ${ }^{104}$ B. Asavapibhop, ${ }^{105}$ N. Srimanobhas, ${ }^{105}$ N. Suwonjandee, ${ }^{105}$ A. Adiguzel, ${ }^{106}$ M. N. Bakirci, ${ }^{106, n n}$ S. Cerci, ${ }^{106,00}$ C. Dozen, ${ }^{106}$ I. Dumanoglu, ${ }^{106}$ E. Eskut, ${ }^{106}$ S. Girgis, ${ }^{106}$ G. Gokbulut, ${ }^{106}$ E. Gurpinar, ${ }^{106}$ I. Hos, ${ }^{106}$ E. E. Kangal, ${ }^{106}$ A. Kayis Topaksu, ${ }^{106}$ G. Onengut, ${ }^{106, p p}$ K. Ozdemir, ${ }^{106}$ S. Ozturk, ${ }^{106, n n}$ A. Polatoz, ${ }^{106}$ K. Sogut, ${ }^{106, q q}$ D. Sunar Cerci, ${ }^{106,00}$ B. Tali, ${ }^{106,00}$ H. Topakli, ${ }^{106, n n}$ M. Vergili, ${ }^{106}$ I. V. Akin, ${ }^{107}$ B. Bilin, ${ }^{107}$ S. Bilmis, ${ }^{107}$ H. Gamsizkan, ${ }^{107}$ G. Karapinar, ${ }^{107, r r}$ K. Ocalan, ${ }^{107}$ U. E. Surat, ${ }^{107}$ M. Yalvac, ${ }^{107}$ M. Zeyrek, ${ }^{107}$ E. Gülmez, ${ }^{108}$ B. Isildak, ${ }^{108, \text { ss }}$ M. Kaya, ${ }^{108, t t}$ O. Kaya, ${ }^{108, t t}$ H. Bahtiyar, ${ }^{109, \text { uu }}$ E. Barlas, ${ }^{109}$ K. Cankocak, ${ }^{109}$ F. I. Vardarl1 ${ }^{109}$ M. Yücel, ${ }^{109}$ L. Levchuk, ${ }^{110}$ P. Sorokin, ${ }^{110}$ J. J. Brooke, ${ }^{111}$ E. Clement,${ }^{111}$ D. Cussans, ${ }^{111}$ H. Flacher, ${ }^{111}$ R. Frazier, ${ }^{111}$ J. Goldstein,${ }^{111}$ M. Grimes, ${ }^{111}$ G. P. Heath,${ }^{111}$ H. F. Heath, ${ }^{111}$ J. Jacob,${ }^{111}$ L. Kreczko, ${ }^{111}$ C. Lucas, ${ }^{111}$ Z. Meng, ${ }^{111}$ D. M. Newbold, ${ }^{11, \text { vv }}$ S. Paramesvaran, ${ }^{111}$ A. Poll, ${ }^{111}$ S. Senkin, ${ }^{111}$ V. J. Smith, ${ }^{111}$ T. Williams, ${ }^{111}$ K. W. Bell, ${ }^{112}$ A. Belyaev, ${ }^{112, w w}$ C. Brew, ${ }^{112}$ R. M. Brown, ${ }^{112}$ D. J. A. Cockerill, ${ }^{112}$ J. A. Coughlan, ${ }^{112}$ K. Harder, ${ }^{112}$ S. Harper, ${ }^{112}$ E. Olaiya, ${ }^{112}$ D. Petyt, ${ }^{112}$ C. H. Shepherd-Themistocleous, ${ }^{112}$ A. Thea,${ }^{112}$ I. R. Tomalin, ${ }^{112}$ W. J. Womersley, ${ }^{112}$ S. D. Worm, ${ }^{112}$ M. Baber, ${ }^{113}$ R. Bainbridge, ${ }^{113}$ O. Buchmuller, ${ }^{113}$ D. Burton, ${ }^{113}$ D. Colling, ${ }^{113}$ N. Cripps,${ }^{113}$ M. Cutajar, ${ }^{113}$ P. Dauncey, ${ }^{113}$ G. Davies, ${ }^{113}$ M. Della Negra, ${ }^{113}$ P. Dunne, ${ }^{113}$ W. Ferguson, ${ }^{113}$ J. Fulcher, ${ }^{113}$ D. Futyan, ${ }^{113}$ A. Gilbert, ${ }^{113}$ 
G. Hall, ${ }^{113}$ G. Iles, ${ }^{113}$ M. Jarvis, ${ }^{113}$ G. Karapostoli, ${ }^{113}$ M. Kenzie, ${ }^{113}$ R. Lane, ${ }^{113}$ R. Lucas, ${ }^{113, v v}$ L. Lyons, ${ }^{113}$ A.-M. Magnan, ${ }^{113}$ S. Malik, ${ }^{113}$ B. Mathias ${ }^{113}$ J. Nash,${ }^{113}$ A. Nikitenko, ${ }^{113,11}$ J. Pela, ${ }^{113}$ M. Pesaresi, ${ }^{113}$ K. Petridis, ${ }^{113}$ D. M. Raymond ${ }^{113}$ S. Rogerson, ${ }^{113}$ A. Rose, ${ }^{113}$ C. Seez, ${ }^{113}$ P. Sharp, ${ }^{113, a}$ A. Tapper, ${ }^{113}$ M. Vazquez Acosta, ${ }^{113}$ T. Virdee, ${ }^{113}$ J. E. Cole, ${ }^{114}$ P. R. Hobson, ${ }^{114}$ A. Khan, ${ }^{114}$ P. Kyberd, ${ }^{114}$ D. Leggat, ${ }^{114}$ D. Leslie, ${ }^{114}$ W. Martin, ${ }^{114}$ I. D. Reid,${ }^{114}$

P. Symonds, ${ }^{114}$ L. Teodorescu, ${ }^{114}$ M. Turner, ${ }^{114}$ J. Dittmann, ${ }^{115}$ K. Hatakeyama,${ }^{115}$ A. Kasmi, ${ }^{115}$ H. Liu, ${ }^{115}$

T. Scarborough, ${ }^{115}$ O. Charaf,${ }^{116}$ S. I. Cooper,${ }^{116}$ C. Henderson, ${ }^{116}$ P. Rumerio, ${ }^{116}$ A. Avetisyan, ${ }^{117}$ T. Bose,${ }^{117}$ C. Fantasia, ${ }^{117}$ A. Heister ${ }^{117}$ P. Lawson, ${ }^{117}$ C. Richardson, ${ }^{117}$ J. Rohlf, ${ }^{117}$ D. Sperka, ${ }^{117}$ J. St. John, ${ }^{117}$ L. Sulak, ${ }^{117}$ J. Alimena, ${ }^{118}$ S. Bhattacharya, ${ }^{118}$ G. Christopher, ${ }^{118}$ D. Cutts, ${ }^{118}$ Z. Demiragli, ${ }^{18}$ A. Ferapontov, ${ }^{118}$ A. Garabedian, ${ }^{118}$ U. Heintz, ${ }^{118}$ S. Jabeen, ${ }^{118}$ G. Kukartsev, ${ }^{118}$ E. Laird ${ }^{118}$ G. Landsberg, ${ }^{118}$ M. Luk, ${ }^{118}$ M. Narain, ${ }^{118}$ M. Segala, ${ }^{118}$ T. Sinthuprasith, ${ }^{118}$ T. Speer, ${ }^{118}$ J. Swanson, ${ }^{118}$ R. Breedon, ${ }^{119}$ G. Breto, ${ }^{119}$ M. Calderon De La Barca Sanchez, ${ }^{119}$ S. Chauhan, ${ }^{119}$ M. Chertok, ${ }^{119}$ J. Conway, ${ }^{119}$ R. Conway, ${ }^{119}$ P. T. Cox,${ }^{119}$ R. Erbacher, ${ }^{119}$ M. Gardner, ${ }^{119}$ W. Ko, ${ }^{119}$ R. Lander, ${ }^{119}$ T. Miceli, ${ }^{119}$ M. Mulhearn, ${ }^{119}$ D. Pellett, ${ }^{119}$ J. Pilot, ${ }^{119}$ F. Ricci-Tam, ${ }^{119}$ M. Searle, ${ }^{119}$ S. Shalhout, ${ }^{119}$ J. Smith, ${ }^{119}$ M. Squires,${ }^{119}$ D. Stolp, ${ }^{119}$ M. Tripathi, ${ }^{119}$ S. Wilbur, ${ }^{119}$ R. Yohay, ${ }^{119}$ R. Cousins, ${ }^{120}$ P. Everaerts, ${ }^{120}$ C. Farrell,,${ }^{120}$ J. Hauser, ${ }^{120}$ M. Ignatenko, ${ }^{120}$ G. Rakness, ${ }^{120}$ E. Takasugi, ${ }^{120}$ V. Valuev, ${ }^{120}$ M. Weber, ${ }^{120} \mathrm{~J}$. Babb, ${ }^{121}$ R. Clare, ${ }^{121} \mathrm{~J}$. Ellison, ${ }^{121}$ J. W. Gary, ${ }^{121}$ G. Hanson, ${ }^{121}$ J. Heilman, ${ }^{121}$ M. Ivova Rikova, ${ }^{121}$ P. Jandir, ${ }^{121}$ E. Kennedy, ${ }^{121}$ F. Lacroix, ${ }^{121}$ H. Liu, ${ }^{121}$ O. R. Long, ${ }^{121}$ A. Luthra, ${ }^{121}$ M. Malberti, ${ }^{121}$ H. Nguyen, ${ }^{121}$ A. Shrinivas, ${ }^{121}$ S. Sumowidagdo, ${ }^{121}$ S. Wimpenny, ${ }^{121}$ W. Andrews, ${ }^{122}$ J. G. Branson, ${ }^{122}$ G. B. Cerati, ${ }^{122}$ S. Cittolin, ${ }^{122}$ R. T. D’Agnolo, ${ }^{122}$ D. Evans, ${ }^{122}$ A. Holzner, ${ }^{122}$ R. Kelley, ${ }^{122}$ D. Klein, ${ }^{122}$ M. Lebourgeois, ${ }^{122}$ J. Letts, ${ }^{122}$ I. Macneill, ${ }^{122}$ D. Olivito, ${ }^{122}$ S. Padhi, ${ }^{122}$ C. Palmer, ${ }^{122}$ M. Pieri, ${ }^{122}$ M. Sani, ${ }^{122}$ V. Sharma, ${ }^{122}$ S. Simon, ${ }^{122}$ E. Sudano, ${ }^{122}$ M. Tadel, ${ }^{122}$ Y. Tu, ${ }^{122}$ A. Vartak, ${ }^{122}$ C. Welke, ${ }^{122}$ F. Würthwein, ${ }^{122}$ A. Yagil, ${ }^{122}$

J. Yoo, ${ }^{122}$ D. Barge, ${ }^{123}$ J. Bradmiller-Feld, ${ }^{123}$ C. Campagnari, ${ }^{123}$ T. Danielson, ${ }^{123}$ A. Dishaw, ${ }^{123}$ K. Flowers,${ }^{123}$ M. Franco Sevilla, ${ }^{123}$ P. Geffert, ${ }^{123}$ C. George,${ }^{123}$ F. Golf, ${ }^{123}$ L. Gouskos, ${ }^{123}$ J. Incandela, ${ }^{123}$ C. Justus,${ }^{123}$ N. Mccoll, ${ }^{123}$ J. Richman, ${ }^{123}$ D. Stuart, ${ }^{123}$ W. To, ${ }^{123}$ C. West, ${ }^{123}$ A. Apresyan, ${ }^{124}$ A. Bornheim, ${ }^{124}$ J. Bunn, ${ }^{124}$ Y. Chen, ${ }^{124}$ E. Di Marco, ${ }^{124}$ J. Duarte, ${ }^{124}$ A. Mott, ${ }^{124}$ H. B. Newman, ${ }^{124}$ C. Pena, ${ }^{124}$ C. Rogan,,${ }^{124}$ M. Spiropulu, ${ }^{124}$ V. Timciuc,,${ }^{124}$ R. Wilkinson, ${ }^{124}$ S. Xie, ${ }^{124}$ R. Y. Zhu, ${ }^{124}$ V. Azzolini, ${ }^{125}$ A. Calamba, ${ }^{125}$ T. Ferguson, ${ }^{125}$ Y. Iiyama, ${ }^{125}$ M. Paulini, ${ }^{125}$ J. Russ, ${ }^{125}$ H. Vogel, ${ }^{125}$ I. Vorobiev, ${ }^{125}$ J. P. Cumalat, ${ }^{126}$ B. R. Drell, ${ }^{126}$ W. T. Ford, ${ }^{126}$ A. Gaz, ${ }^{126}$ E. Luiggi Lopez, ${ }^{126}$ U. Nauenberg, ${ }^{126}$ J. G. Smith, ${ }^{126}$ K. Stenson, ${ }^{126}$ K. A. Ulmer, ${ }^{126}$ S. R. Wagner, ${ }^{126}$ J. Alexander, ${ }^{127}$ A. Chatterjee, ${ }^{127}$ J. Chu, ${ }^{127}$ S. Dittmer, ${ }^{127}$ N. Eggert, ${ }^{127}$ N. Mirman, ${ }^{127}$ G. Nicolas Kaufman, ${ }^{127}$ J. R. Patterson, ${ }^{127}$ A. Ryd, ${ }^{127}$ E. Salvati, ${ }^{127}$ L. Skinnari, ${ }^{127}$ W. Sun, ${ }^{127}$ W. D. Teo, ${ }^{127}$

J. Thom, ${ }^{127}$ J. Thompson, ${ }^{127}$ J. Tucker, ${ }^{127}$ Y. Weng, ${ }^{127}$ L. Winstrom, ${ }^{127}$ P. Wittich, ${ }^{127}$ D. Winn, ${ }^{128}$ S. Abdullin, ${ }^{129}$ M. Albrow, ${ }^{129}$ J. Anderson, ${ }^{129}$ G. Apollinari, ${ }^{129}$ L. A. T. Bauerdick, ${ }^{129}$ A. Beretvas, ${ }^{129}$ J. Berryhill, ${ }^{129}$ P. C. Bhat, ${ }^{129}$ K. Burkett, ${ }^{129}$ J. N. Butler, ${ }^{129}$ H. W. K. Cheung, ${ }^{129}$ F. Chlebana, ${ }^{129}$ S. Cihangir, ${ }^{129}$ V. D. Elvira, ${ }^{129}$ I. Fisk, ${ }^{129}$ J. Freeman, ${ }^{129}$

Y. Gao, ${ }^{129}$ E. Gottschalk, ${ }^{129}$ L. Gray, ${ }^{129}$ D. Green, ${ }^{129}$ S. Grünendahl, ${ }^{129}$ O. Gutsche, ${ }^{129}$ J. Hanlon, ${ }^{129}$ D. Hare, ${ }^{129}$

R. M. Harris, ${ }^{129}$ J. Hirschauer, ${ }^{129}$ B. Hooberman, ${ }^{129}$ S. Jindariani, ${ }^{129}$ M. Johnson, ${ }^{129}$ U. Joshi, ${ }^{129}$ K. Kaadze, ${ }^{129}$ B. Klima, ${ }^{129}$

B. Kreis, ${ }^{129}$ S. Kwan, ${ }^{129}$ J. Linacre, ${ }^{129}$ D. Lincoln, ${ }^{129}$ R. Lipton, ${ }^{129}$ T. Liu, ${ }^{129}$ J. Lykken, ${ }^{129}$ K. Maeshima, ${ }^{129}$

J. M. Marraffino, ${ }^{129}$ V. I. Martinez Outschoorn, ${ }^{129}$ S. Maruyama, ${ }^{129}$ D. Mason, ${ }^{129}$ P. McBride, ${ }^{129}$ K. Mishra,${ }^{129}$ S. Mrenna, ${ }^{129}$ Y. Musienko, ${ }^{129, e \mathrm{ee}}$ S. Nahn, ${ }^{129}$ C. Newman-Holmes, ${ }^{129}$ V. O’Dell, ${ }^{129}$ O. Prokofyev, ${ }^{129}$ E. Sexton-Kennedy, ${ }^{129}$ S. Sharma, ${ }^{129}$ A. Soha, ${ }^{129}$ W. J. Spalding, ${ }^{129}$ L. Spiegel, ${ }^{129}$ L. Taylor, ${ }^{129}$ S. Tkaczyk, ${ }^{129}$ N. V. Tran, ${ }^{129}$ L. Uplegger, ${ }^{129}$ E. W. Vaandering, ${ }^{129}$ R. Vidal, ${ }^{129}$ A. Whitbeck, ${ }^{129}$ J. Whitmore, ${ }^{129}$ F. Yang, ${ }^{129}$ D. Acosta,${ }^{130}$ P. Avery, ${ }^{130}$ D. Bourilkov, ${ }^{130}$ M. Carver, ${ }^{130}$

T. Cheng, ${ }^{130}$ D. Curry, ${ }^{130}$ S. Das, ${ }^{130}$ M. De Gruttola, ${ }^{130}$ G. P. Di Giovanni, ${ }^{130}$ R. D. Field, ${ }^{130}$ M. Fisher, ${ }^{130}$ I. K. Furic, ${ }^{130}$ J. Hugon, ${ }^{130}$ J. Konigsberg, ${ }^{130}$ A. Korytov, ${ }^{130}$ T. Kypreos, ${ }^{130}$ J. F. Low, ${ }^{130}$ K. Matchev, ${ }^{130}$ P. Milenovic, ${ }^{130, x x}$ G. Mitselmakher, ${ }^{130}$ L. Muniz, ${ }^{130}$ A. Rinkevicius, ${ }^{130}$ L. Shchutska, ${ }^{130}$ N. Skhirtladze, ${ }^{130}$ M. Snowball, ${ }^{130}$ J. Yelton, ${ }^{130}$ M. Zakaria, ${ }^{130}$ S. Hewamanage, ${ }^{131}$ S. Linn, ${ }^{131}$ P. Markowitz, ${ }^{131}$ G. Martinez, ${ }^{131}$ J. L. Rodriguez, ${ }^{131}$ T. Adams, ${ }^{132}$ A. Askew, ${ }^{132}$ J. Bochenek, ${ }^{132}$ B. Diamond, ${ }^{132}$ J. Haas, ${ }^{132}$ S. Hagopian, ${ }^{132}$ V. Hagopian, ${ }^{132}$ K. F. Johnson, ${ }^{132}$ H. Prosper, ${ }^{132}$ V. Veeraraghavan, ${ }^{132}$ M. Weinberg, ${ }^{132}$ M. M. Baarmand, ${ }^{133}$ M. Hohlmann, ${ }^{133}$ H. Kalakhety, ${ }^{133}$ F. Yumiceva, ${ }^{133}$ M. R. Adams, ${ }^{134}$ L. Apanasevich, ${ }^{134}$ V. E. Bazterra, ${ }^{134}$ D. Berry ${ }^{134}$ R. R. Betts, ${ }^{134}$ I. Bucinskaite,${ }^{134}$ R. Cavanaugh, ${ }^{134}$ O. Evdokimov, ${ }^{134}$ L. Gauthier, ${ }^{134}$ C. E. Gerber, ${ }^{134}$ D. J. Hofman, ${ }^{134}$ S. Khalatyan, ${ }^{134}$ P. Kurt, ${ }^{134}$ D. H. Moon, ${ }^{134}$ C. O'Brien, ${ }^{134}$ C. Silkworth, ${ }^{134}$ P. Turner, ${ }^{134}$ N. Varelas, ${ }^{134}$ E. A. Albayrak,,${ }^{135, \text { uu }}$ B. Bilki, ${ }^{135, y y}$ W. Clarida, ${ }^{135}$ K. Dilsiz, ${ }^{135}$ F. Duru, ${ }^{135}$ M. Haytmyradov, ${ }^{135}$ J.-P. Merlo, ${ }^{135}$ H. Mermerkaya, ${ }^{135, z z}$ A. Mestvirishvili, ${ }^{135}$ A. Moeller, ${ }^{135}$ J. Nachtman, ${ }^{135}$ H. Ogul, ${ }^{135}$ Y. Onel, ${ }^{135}$ F. Ozok, ${ }^{135, \text { uu }}$ A. Penzo, ${ }^{135}$ R. Rahmat, ${ }^{135}$ S. Sen, ${ }^{135}$ P. Tan,${ }^{135}$ E. Tiras,${ }^{135}$ J. Wetzel,,${ }^{135}$ T. Yetkin, ${ }^{135, \text { aaa }}$ K. Yi, ${ }^{135}$ B. A. Barnett, ${ }^{136}$ B. Blumenfeld, ${ }^{136}$ S. Bolognesi, ${ }^{136}$ D. Fehling, ${ }^{136}$ A. V. Gritsan, ${ }^{136}$ 
P. Maksimovic ${ }^{136}$ C. Martin, ${ }^{136}$ M. Swartz, ${ }^{136}$ P. Baringer, ${ }^{137}$ A. Bean ${ }^{137}$ G. Benelli, ${ }^{137}$ C. Bruner, ${ }^{137}$ J. Gray, ${ }^{137}$ R. P. Kenny III ${ }^{137}$ M. Murray, ${ }^{137}$ D. Noonan, ${ }^{137}$ S. Sanders, ${ }^{137}$ J. Sekaric,${ }^{137}$ R. Stringer, ${ }^{137}$ Q. Wang,${ }^{137}$ J. S. Wood, ${ }^{137}$ A. F. Barfuss, ${ }^{138}$ I. Chakaberia, ${ }^{138}$ A. Ivanov, ${ }^{138}$ S. Khalil, ${ }^{138}$ M. Makouski, ${ }^{138}$ Y. Maravin, ${ }^{138}$ L. K. Saini, ${ }^{138}$ S. Shrestha, ${ }^{138}$ I. Svintradze, ${ }^{138}$ J. Gronberg, ${ }^{139}$ D. Lange, ${ }^{139}$ F. Rebassoo, ${ }^{139}$ D. Wright, ${ }^{139}$ A. Baden, ${ }^{140}$ A. Belloni, ${ }^{140}$ B. Calvert, ${ }^{140}$ S. C. Eno, ${ }^{140}$ J. A. Gomez, ${ }^{140}$ N. J. Hadley, ${ }^{140}$ R. G. Kellogg, ${ }^{140}$ T. Kolberg, ${ }^{140}$ Y. Lu, ${ }^{140}$ M. Marionneau, ${ }^{140}$

A. C. Mignerey ${ }^{140}$ K. Pedro, ${ }^{140}$ A. Skuja, ${ }^{140}$ M. B. Tonjes, ${ }^{140}$ S. C. Tonwar, ${ }^{140}$ A. Apyan, ${ }^{141}$ R. Barbieri, ${ }^{141}$ G. Bauer, ${ }^{141}$ W. Busza, ${ }^{141}$ I. A. Cali, ${ }^{141}$ M. Chan,${ }^{141}$ L. Di Matteo,${ }^{141}$ V. Dutta,${ }^{141}$ G. Gomez Ceballos, ${ }^{141}$ M. Goncharov, ${ }^{141}$ D. Gulhan, ${ }^{141}$ M. Klute, ${ }^{141}$ Y. S. Lai, ${ }^{141}$ Y.-J. Lee, ${ }^{141}$ A. Levin, ${ }^{141}$ P. D. Luckey,${ }^{141}$ T. Ma,${ }^{141}$ C. Paus, ${ }^{141}$ D. Ralph,${ }^{141}$ C. Roland,${ }^{141}$ G. Roland, ${ }^{141}$ G. S. F. Stephans, ${ }^{141}$ F. Stöckli, ${ }^{141}$ K. Sumorok,${ }^{141}$ D. Velicanu,${ }^{141}$ J. Veverka, ${ }^{141}$ B. Wyslouch,${ }^{141}$ M. Yang, ${ }^{141}$ M. Zanetti, ${ }^{141}$ V. Zhukova, ${ }^{141}$ B. Dahmes, ${ }^{142}$ A. Gude ${ }^{142}$ S. C. Kao, ${ }^{142}$ K. Klapoetke, ${ }^{142}$ Y. Kubota, ${ }^{142}$ J. Mans, ${ }^{142}$ N. Pastika, ${ }^{142}$ R. Rusack, ${ }^{142}$ A. Singovsky, ${ }^{142}$ N. Tambe, ${ }^{142}$ J. Turkewitz, ${ }^{142}$ J. G. Acosta, ${ }^{143}$ S. Oliveros, ${ }^{143}$ E. Avdeeva, ${ }^{144}$ K. Bloom, ${ }^{144}$ S. Bose,${ }^{144}$ D. R. Claes ${ }^{144}$ A. Dominguez, ${ }^{144}$ R. Gonzalez Suarez, ${ }^{144}$ J. Keller, ${ }^{144}$ D. Knowlton, ${ }^{144}$ I. Kravchenko, ${ }^{144}$ J. Lazo-Flores ${ }^{144}$ S. Malik, ${ }^{144}$ F. Meier, ${ }^{144}$ G. R. Snow, ${ }^{144}$ J. Dolen, ${ }^{145}$ A. Godshalk, ${ }^{145}$ I. Iashvili, ${ }^{145}$ A. Kharchilava, ${ }^{145}$ A. Kumar, ${ }^{145}$ S. Rappoccio, ${ }^{145}$ G. Alverson, ${ }^{146}$ E. Barberis, ${ }^{146}$ D. Baumgartel, ${ }^{146}$ M. Chasco, ${ }^{146}$ J. Haley, ${ }^{146}$ A. Massironi, ${ }^{146}$ D. M. Morse, ${ }^{146}$ D. Nash,${ }^{146}$ T. Orimoto, ${ }^{146}$ D. Trocino, ${ }^{146}$ R. J. Wang, ${ }^{146}$ D. Wood, ${ }^{146}$ J. Zhang, ${ }^{146}$ K. A. Hahn, ${ }^{147}$ A. Kubik,${ }^{147}$ N. Mucia, ${ }^{147}$ N. Odell, ${ }^{147}$ B. Pollack, ${ }^{147}$ A. Pozdnyakov, ${ }^{147}$ M. Schmitt, ${ }^{147}$ S. Stoynev, ${ }^{147}$ K. Sung, ${ }^{147}$ M. Velasco, ${ }^{147}$ S. Won, ${ }^{147}$ A. Brinkerhoff, ${ }^{148}$ K. M. Chan, ${ }^{148}$ A. Drozdetskiy, ${ }^{148}$ M. Hildreth, ${ }^{148}$ C. Jessop, ${ }^{148}$ D. J. Karmgard, ${ }^{148}$ N. Kellams, ${ }^{148}$ K. Lannon, ${ }^{148}$ W. Luo, ${ }^{148}$ S. Lynch, ${ }^{148}$ N. Marinelli, ${ }^{148}$ T. Pearson,,${ }^{148}$ M. Planer,${ }^{148}$ R. Ruchti, ${ }^{148}$ N. Valls, ${ }^{148}$ M. Wayne, ${ }^{148}$ M. Wolf, ${ }^{148}$ A. Woodard ${ }^{148}$ L. Antonelli, ${ }^{149}$ J. Brinson, ${ }^{149}$ B. Bylsma, ${ }^{149}$ L. S. Durkin, ${ }^{149}$ S. Flowers, ${ }^{149}$ C. Hill, ${ }^{149}$ R. Hughes,${ }^{149}$ K. Kotov,${ }^{149}$ T. Y. Ling, ${ }^{149}$ D. Puigh,${ }^{149}$ M. Rodenburg, ${ }^{149}$ G. Smith, ${ }^{149}$ C. Vuosalo, ${ }^{149}$ B. L. Winer, ${ }^{149}$ H. Wolfe ${ }^{149}$ H. W. Wulsin, ${ }^{149}$ E. Berry, ${ }^{150}$ O. Driga, ${ }^{150}$ P. Elmer, ${ }^{150}$ P. Hebda,${ }^{150}$ A. Hunt,,${ }^{150}$ S. A. Koay, ${ }^{150}$ P. Lujan, ${ }^{150}$ D. Marlow, ${ }^{150}$ T. Medvedeva, ${ }^{150}$ M. Mooney, ${ }^{150}$ J. Olsen,,${ }^{150}$ P. Piroué, ${ }^{150}$ X. Quan, ${ }^{150}$ H. Saka, ${ }^{150}$ D. Stickland,${ }^{150, c}$ C. Tully, ${ }^{150}$ J. S. Werner, ${ }^{150}$ S. C. Zenz, ${ }^{150}$ A. Zuranski, ${ }^{150}$ E. Brownson, ${ }^{151}$ H. Mendez,${ }^{151}$ J. E. Ramirez Vargas, ${ }^{151}$ E. Alagoz,${ }^{152}$ V. E. Barnes,${ }^{152}$ D. Benedetti, ${ }^{152}$ G. Bolla, ${ }^{152}$ D. Bortoletto, ${ }^{152}$ M. De Mattia, ${ }^{152}$ Z. Hu, ${ }^{152}$ M. K. Jha, ${ }^{152}$ M. Jones, ${ }^{152}$ K. Jung, ${ }^{152}$ M. Kress,${ }^{152}$ N. Leonardo, ${ }^{152}$ D. Lopes Pegna, ${ }^{152}$ V. Maroussov, ${ }^{152}$ P. Merkel, ${ }^{152}$ D. H. Miller, ${ }^{152}$ N. Neumeister, ${ }^{152}$ B. C. Radburn-Smith, ${ }^{152}$ X. Shi, ${ }^{152}$ I. Shipsey, ${ }^{152}$ D. Silvers, ${ }^{152}$ A. Svyatkovskiy, ${ }^{152}$ F. Wang, ${ }^{152}$ W. Xie, ${ }^{152}$ L. Xu,${ }^{152}$ H. D. Yoo, ${ }^{152}$ J. Zablocki, ${ }^{152}$ Y. Zheng, ${ }^{152}$ N. Parashar, ${ }^{153}$ J. Stupak, ${ }^{153}$ A. Adair, ${ }^{154}$ B. Akgun, ${ }^{154}$ K. M. Ecklund, ${ }^{154}$ F. J. M. Geurts, ${ }^{154}$ W. Li ${ }^{154}$ B. Michlin, ${ }^{154}$ B. P. Padley, ${ }^{154}$ R. Redjimi, ${ }^{154}$ J. Roberts, ${ }^{154}$ J. Zabel, ${ }^{154}$ B. Betchart, ${ }^{155}$ A. Bodek, ${ }^{155}$ R. Covarelli, ${ }^{155}$ P. de Barbaro, ${ }^{155}$ R. Demina, ${ }^{155}$ Y. Eshaq, ${ }^{155}$ T. Ferbel, ${ }^{155}$ A. Garcia-Bellido, ${ }^{155}$ P. Goldenzweig ${ }^{155}$ J. Han ${ }^{155}$ A. Harel, ${ }^{155}$

A. Khukhunaishvili, ${ }^{155}$ D. C. Miner, ${ }^{155}$ G. Petrillo, ${ }^{155}$ D. Vishnevskiy, ${ }^{155}$ R. Ciesielski, ${ }^{156}$ L. Demortier, ${ }^{156}$ K. Goulianos, ${ }^{156}$ G. Lungu, ${ }^{156}$ C. Mesropian, ${ }^{156}$ S. Arora, ${ }^{157}$ A. Barker, ${ }^{157}$ J. P. Chou, ${ }^{157}$ C. Contreras-Campana, ${ }^{157}$ E. Contreras-Campana, ${ }^{157}$ D. Duggan, ${ }^{157}$ D. Ferencek, ${ }^{157}$ Y. Gershtein, ${ }^{157}$ R. Gray, ${ }^{157}$ E. Halkiadakis, ${ }^{157}$ D. Hidas, ${ }^{157}$ A. Lath, ${ }^{157}$ S. Panwalkar, ${ }^{157}$ M. Park, ${ }^{157}$ R. Patel, ${ }^{157}$ V. Rekovic, ${ }^{157}$ S. Salur, ${ }^{157}$ S. Schnetzer, ${ }^{157}$ C. Seitz, ${ }^{157}$ S. Somalwar, ${ }^{157}$ R. Stone,${ }^{157}$ S. Thomas, ${ }^{157}$ P. Thomassen, ${ }^{157}$ M. Walker, ${ }^{157}$ K. Rose,${ }^{158}$ S. Spanier, ${ }^{158}$ A. York, ${ }^{158}$ O. Bouhali, ${ }^{159, b b b}$ R. Eusebi, ${ }^{159}$ W. Flanagan,${ }^{159}$ J. Gilmore, ${ }^{159}$ T. Kamon, ${ }^{159, \text { ccc }}$ V. Khotilovich, ${ }^{159}$ V. Krutelyov, ${ }^{159}$ R. Montalvo, ${ }^{159}$ I. Osipenkov, ${ }^{159}$ Y. Pakhotin, ${ }^{159}$

A. Perloff, ${ }^{159}$ J. Roe, ${ }^{159}$ A. Rose, ${ }^{159}$ A. Safonov, ${ }^{159}$ T. Sakuma, ${ }^{159}$ I. Suarez, ${ }^{159}$ A. Tatarinov, ${ }^{159}$ N. Akchurin, ${ }^{160}$ C. Cowden ${ }^{160}$ J. Damgov, ${ }^{160}$ C. Dragoiu, ${ }^{160}$ P. R. Dudero, ${ }^{160}$ J. Faulkner, ${ }^{160}$ K. Kovitanggoon, ${ }^{160}$ S. Kunori,${ }^{160}$ S. W. Lee, ${ }^{160}$ T. Libeiro, ${ }^{160}$ I. Volobouev, ${ }^{160}$ E. Appelt, ${ }^{161}$ A. G. Delannoy, ${ }^{161}$ S. Greene, ${ }^{161}$ A. Gurrola, ${ }^{161}$ W. Johns,${ }^{161}$ C. Maguire, ${ }^{161}$ Y. Mao, ${ }^{161}$ A. Melo, ${ }^{161}$ M. Sharma, ${ }^{161}$ P. Sheldon, ${ }^{161}$ B. Snook, ${ }^{161}$ S. Tuo, ${ }^{161}$ J. Velkovska, ${ }^{161}$ M. W. Arenton, ${ }^{162}$ S. Boutle, ${ }^{162}$ B. Cox ${ }^{162}$ B. Francis, ${ }^{162}$ J. Goodell, ${ }^{162}$ R. Hirosky, ${ }^{162}$ A. Ledovskoy, ${ }^{162}$ H. Li,${ }^{162}$ C. Lin, ${ }^{162}$ C. Neu, ${ }^{162}$ J. Wood, ${ }^{162}$

S. Gollapinni, ${ }^{163}$ R. Harr, ${ }^{163}$ P. E. Karchin, ${ }^{163}$ C. Kottachchi Kankanamge Don, ${ }^{163}$ P. Lamichhane, ${ }^{163}$ J. Sturdy, ${ }^{163}$ D. A. Belknap, ${ }^{164}$ D. Carlsmith, ${ }^{164}$ M. Cepeda, ${ }^{164}$ S. Dasu, ${ }^{164}$ S. Duric, ${ }^{164}$ E. Friis, ${ }^{164}$ R. Hall-Wilton, ${ }^{164}$ M. Herndon, ${ }^{164}$ A. Hervé, ${ }^{164}$ P. Klabbers, ${ }^{164}$ A. Lanaro, ${ }^{164}$ C. Lazaridis, ${ }^{164}$ A. Levine, ${ }^{164}$ R. Loveless, ${ }^{164}$ A. Mohapatra, ${ }^{164}$ I. Ojalvo, ${ }^{164}$ T. Perry, ${ }^{164}$ G. A. Pierro, ${ }^{164}$ G. Polese, ${ }^{164}$ I. Ross, ${ }^{164}$ T. Sarangi, ${ }^{164}$ A. Savin, ${ }^{164}$ W. H. Smith, ${ }^{164}$ and N. Woods ${ }^{164}$

(CMS Collaboration)

\author{
${ }^{1}$ Yerevan Physics Institute, Yerevan, Armenia \\ ${ }^{2}$ Institut für Hochenergiephysik der OeAW, Wien, Austria
}


${ }^{3}$ National Centre for Particle and High Energy Physics, Minsk, Belarus

${ }^{4}$ Universiteit Antwerpen, Antwerpen, Belgium

${ }^{5}$ Vrije Universiteit Brussel, Brussel, Belgium

${ }^{6}$ Université Libre de Bruxelles, Bruxelles, Belgium

${ }^{7}$ Ghent University, Ghent, Belgium

${ }^{8}$ Université Catholique de Louvain, Louvain-la-Neuve, Belgium

${ }^{9}$ Université de Mons, Mons, Belgium

${ }^{10}$ Centro Brasileiro de Pesquisas Fisicas, Rio de Janeiro, Brazil

${ }^{11}$ Universidade do Estado do Rio de Janeiro, Rio de Janeiro, Brazil

${ }^{12 a}$ Universidade Estadual Paulista, São Paulo, Brazil

${ }^{12 \mathrm{~b}}$ Universidade Federal do ABC, São Paulo, Brazil

${ }^{13}$ Institute for Nuclear Research and Nuclear Energy, Sofia, Bulgaria

${ }^{14}$ University of Sofia, Sofia, Bulgaria

${ }^{15}$ Institute of High Energy Physics, Beijing, China

${ }^{16}$ State Key Laboratory of Nuclear Physics and Technology, Peking University, Beijing, China

${ }^{17}$ Universidad de Los Andes, Bogota, Colombia

${ }^{18}$ University of Split, Faculty of Electrical Engineering, Mechanical Engineering and Naval Architecture, Split, Croatia

${ }^{19}$ University of Split, Faculty of Science, Split, Croatia

${ }^{20}$ Institute Rudjer Boskovic, Zagreb, Croatia

${ }^{21}$ University of Cyprus, Nicosia, Cyprus

${ }^{22}$ Charles University, Prague, Czech Republic

${ }^{23}$ Academy of Scientific Research and Technology of the Arab Republic of Egypt,

Egyptian Network of High Energy Physics, Cairo, Egypt

${ }^{24}$ National Institute of Chemical Physics and Biophysics, Tallinn, Estonia

${ }^{25}$ Department of Physics, University of Helsinki, Helsinki, Finland

${ }^{26}$ Helsinki Institute of Physics, Helsinki, Finland

${ }^{27}$ Lappeenranta University of Technology, Lappeenranta, Finland

${ }^{28}$ DSM/IRFU, CEA/Saclay, Gif-sur-Yvette, France

${ }^{29}$ Laboratoire Leprince-Ringuet, Ecole Polytechnique, IN2P3-CNRS, Palaiseau, France

${ }^{30}$ Institut Pluridisciplinaire Hubert Curien, Université de Strasbourg,

Université de Haute Alsace Mulhouse, CNRS/IN2P3, Strasbourg, France

${ }^{31}$ Centre de Calcul de l'Institut National de Physique Nucleaire et de Physique des Particules, CNRS/IN2P3, Villeurbanne, France

${ }^{32}$ Université de Lyon, Université Claude Bernard Lyon 1, CNRS-IN2P3, Institut de Physique Nucléaire de Lyon, Villeurbanne, France

${ }^{33}$ Institute of High Energy Physics and Informatization, Tbilisi State University, Tbilisi, Georgia

${ }^{34}$ RWTH Aachen University, I. Physikalisches Institut, Aachen, Germany

${ }^{35}$ RWTH Aachen University, III. Physikalisches Institut A, Aachen, Germany

${ }^{36}$ RWTH Aachen University, III. Physikalisches Institut B, Aachen, Germany

${ }^{37}$ Deutsches Elektronen-Synchrotron, Hamburg, Germany

${ }^{38}$ University of Hamburg, Hamburg, Germany

${ }^{39}$ Institut für Experimentelle Kernphysik, Karlsruhe, Germany

${ }^{40}$ Institute of Nuclear and Particle Physics (INPP), NCSR Demokritos, Aghia Paraskevi, Greece

${ }^{41}$ University of Athens, Athens, Greece

${ }^{42}$ University of Ioánnina, Ioánnina, Greece

${ }^{43}$ Wigner Research Centre for Physics, Budapest, Hungary

${ }^{44}$ Institute of Nuclear Research ATOMKI, Debrecen, Hungary

${ }^{45}$ University of Debrecen, Debrecen, Hungary

${ }^{46}$ National Institute of Science Education and Research, Bhubaneswar, India

${ }^{47}$ Panjab University, Chandigarh, India

${ }^{48}$ University of Delhi, Delhi, India

${ }^{49}$ Saha Institute of Nuclear Physics, Kolkata, India

${ }^{50}$ Bhabha Atomic Research Centre, Mumbai, India

${ }^{51}$ Tata Institute of Fundamental Research, Mumbai, India

${ }^{52}$ Institute for Research in Fundamental Sciences (IPM), Tehran, Iran

${ }^{53}$ University College Dublin, Dublin, Ireland

${ }^{54 a}$ INFN Sezione di Bari, Bari, Italy

${ }^{54 \mathrm{~b}}$ Università di Bari, Bari, Italy

${ }^{54}$ Politecnico di Bari, Bari, Italy 
${ }^{55 a}$ INFN Sezione di Bologna, Bologna, Italy

${ }^{55 \mathrm{~b}}$ Università di Bologna, Bologna, Italy

${ }^{56 a}$ INFN Sezione di Catania, Catania, Italy

${ }^{56 \mathrm{~b}}$ Università di Catania, Catania, Italy

${ }^{56}$ CSFNSM, Catania, Italy

${ }^{57 \mathrm{a}}$ INFN Sezione di Firenze, Firenze, Italy

${ }^{57 \mathrm{~b}}$ Università di Firenze, Firenze, Italy

${ }^{58}$ INFN Laboratori Nazionali di Frascati, Frascati, Italy

${ }^{59 a}$ INFN Sezione di Genova, Genova, Italy

${ }^{59 \mathrm{~b}}$ Università di Genova, Genova, Italy

${ }^{60 a}$ INFN Sezione di Milano-Bicocca, Milano, Italy

${ }^{60 \mathrm{~b}}$ Università di Milano-Bicocca, Milano, Italy

${ }^{61 a}$ INFN Sezione di Napoli, Napoli, Italy

${ }^{61 \mathrm{~b}}$ Università di Napoli 'Federico II', Napoli, Italy

${ }^{61 \mathrm{c}}$ Università della Basilicata (Potenza), Napoli, Italy

${ }^{61 \mathrm{~d}}$ Università G. Marconi (Roma), Napoli, Italy

${ }^{62 \mathrm{a}}$ INFN Sezione di Padova, Padova, Italy

${ }^{62 \mathrm{~b}}$ Università di Padova, Padova, Italy

${ }^{62 \mathrm{c}}$ Università di Trento (Trento), Padova, Italy

${ }^{63 a}$ INFN Sezione di Pavia, Pavia, Italy

${ }^{63 \mathrm{~b}}$ Università di Pavia, Pavia, Italy

${ }^{64 a}$ INFN Sezione di Perugia, Perugia, Italy

${ }^{64 \mathrm{~b}}$ Università di Perugia, Perugia, Italy

${ }^{65}$ INFN Sezione di Pisa, Pisa, Italy

${ }^{65 b}$ Università di Pisa, Pisa, Italy

${ }^{65 \mathrm{c}}$ Scuola Normale Superiore di Pisa, Pisa, Italy

${ }^{66 a}$ INFN Sezione di Roma, Roma, Italy

${ }^{66 \mathrm{~b}}$ Università di Roma, Roma, Italy

${ }^{67 a}$ INFN Sezione di Torino, Torino, Italy

${ }^{67 b}$ Università di Torino, Torino, Italy

${ }^{67 \mathrm{c}}$ Università del Piemonte Orientale (Novara), Torino, Italy

${ }^{68 \mathrm{a}}$ INFN Sezione di Trieste, Trieste, Italy

${ }^{68 \mathrm{~b}}$ Università di Trieste, Trieste, Italy

${ }^{69}$ Kangwon National University, Chunchon, Korea

${ }^{70}$ Kyungpook National University, Daegu, Korea

${ }^{71}$ Chonnam National University, Institute for Universe and Elementary Particles, Kwangju, Korea

${ }^{72}$ Korea University, Seoul, Korea

${ }^{73}$ University of Seoul, Seoul, Korea

${ }^{74}$ Sungkyunkwan University, Suwon, Korea

${ }^{75}$ Vilnius University, Vilnius, Lithuania

${ }^{76}$ National Centre for Particle Physics, Universiti Malaya, Kuala Lumpur, Malaysia

${ }^{77}$ Centro de Investigacion y de Estudios Avanzados del IPN, Mexico City, Mexico

${ }^{78}$ Universidad Iberoamericana, Mexico City, Mexico

${ }^{79}$ Benemerita Universidad Autonoma de Puebla, Puebla, Mexico

${ }^{80}$ Universidad Autónoma de San Luis Potosí, San Luis Potosí, Mexico

${ }^{81}$ University of Auckland, Auckland, New Zealand

${ }^{82}$ University of Canterbury, Christchurch, New Zealand

${ }^{83}$ National Centre for Physics, Quaid-I-Azam University, Islamabad, Pakistan

${ }^{84}$ National Centre for Nuclear Research, Swierk, Poland

${ }^{85}$ Institute of Experimental Physics, Faculty of Physics, University of Warsaw, Warsaw, Poland

${ }^{86}$ Laboratório de Instrumentação e Física Experimental de Partículas, Lisboa, Portugal

${ }^{87}$ Joint Institute for Nuclear Research, Dubna, Russia

${ }^{88}$ Petersburg Nuclear Physics Institute, Gatchina (St. Petersburg), Russia

${ }^{89}$ Institute for Nuclear Research, Moscow, Russia

${ }^{90}$ Institute for Theoretical and Experimental Physics, Moscow, Russia

${ }^{91}$ P.N. Lebedev Physical Institute, Moscow, Russia

${ }^{92}$ Skobeltsyn Institute of Nuclear Physics, Lomonosov Moscow State University, Moscow, Russia

${ }^{93}$ State Research Center of Russian Federation, Institute for High Energy Physics, Protvino, Russia

${ }^{94}$ University of Belgrade, Faculty of Physics and Vinca Institute of Nuclear Sciences, Belgrade, Serbia

${ }^{95}$ Centro de Investigaciones Energéticas Medioambientales y Tecnológicas (CIEMAT), Madrid, Spain 
${ }^{96}$ Universidad Autónoma de Madrid, Madrid, Spain

${ }^{97}$ Universidad de Oviedo, Oviedo, Spain

${ }^{98}$ Instituto de Física de Cantabria (IFCA), CSIC-Universidad de Cantabria, Santander, Spain

${ }^{99}$ CERN, European Organization for Nuclear Research, Geneva, Switzerland

${ }^{100}$ Paul Scherrer Institut, Villigen, Switzerland

${ }^{101}$ Institute for Particle Physics, ETH Zurich, Zurich, Switzerland

${ }^{102}$ Universität Zürich, Zurich, Switzerland

${ }^{103}$ National Central University, Chung-Li, Taiwan

${ }^{104}$ National Taiwan University (NTU), Taipei, Taiwan

${ }^{105}$ Chulalongkorn University, Faculty of Science, Department of Physics, Bangkok, Thailand

${ }^{106}$ Cukurova University, Adana, Turkey

${ }^{107}$ Middle East Technical University, Physics Department, Ankara, Turkey

${ }^{108}$ Bogazici University, Istanbul, Turkey

${ }^{109}$ Istanbul Technical University, Istanbul, Turkey

${ }^{110}$ National Scientific Center, Kharkov Institute of Physics and Technology, Kharkov, Ukraine

${ }^{111}$ University of Bristol, Bristol, United Kingdom

${ }^{112}$ Rutherford Appleton Laboratory, Didcot, United Kingdom

${ }^{113}$ Imperial College, London, United Kingdom

${ }^{114}$ Brunel University, Uxbridge, United Kingdom

${ }^{115}$ Baylor University, Waco, Texas 76798, USA

${ }^{116}$ The University of Alabama, Tuscaloosa, Alabama 35487, USA

${ }^{117}$ Boston University, Boston, Massachusetts 02215, USA

${ }^{118}$ Brown University, Providence, Rhode Island 02912, USA

${ }^{119}$ University of California, Davis, California 95616, USA

${ }^{120}$ University of California, Los Angeles, California 90095, USA

${ }^{121}$ University of California, Riverside, California 92521, USA

${ }^{122}$ University of California, San Diego, La Jolla, California 92093, USA

${ }^{123}$ University of California, Santa Barbara, California 93106, USA

${ }^{124}$ California Institute of Technology, Pasadena, California 91125, USA

${ }^{125}$ Carnegie Mellon University, Pittsburgh, Pennsylvania 15213, USA

${ }^{126}$ University of Colorado at Boulder, Boulder, Colorado 80309, USA

${ }^{127}$ Cornell University, Ithaca, New York 14853, USA

${ }^{128}$ Fairfield University, Fairfield, Connecticut 06430, USA

${ }^{129}$ Fermi National Accelerator Laboratory, Batavia, Illinois 60510, USA

${ }^{130}$ University of Florida, Gainesville, Florida 32611, USA

${ }^{131}$ Florida International University, Miami, Florida 33199, USA

${ }^{132}$ Florida State University, Tallahassee, Florida 32306, USA

${ }^{133}$ Florida Institute of Technology, Melbourne, Florida 32901, USA

${ }^{134}$ University of Illinois at Chicago (UIC), Chicago, Illinois 60607, USA

${ }^{135}$ The University of Iowa, Iowa City, Iowa 52242, USA

${ }^{136}$ Johns Hopkins University, Baltimore, Maryland 21218, USA

${ }^{137}$ The University of Kansas, Lawrence, Kansas 66045, USA

${ }^{138}$ Kansas State University, Manhattan, Kansas 66506, USA

${ }^{139}$ Lawrence Livermore National Laboratory, Livermore, California 94551, USA

${ }^{140}$ University of Maryland, College Park, Maryland 20742, USA

${ }^{141}$ Massachusetts Institute of Technology, Cambridge, Massachusetts 02139, USA

${ }^{142}$ University of Minnesota, Minneapolis, Minnesota 55455, USA

${ }^{143}$ University of Mississippi, Oxford, Mississippi 38677, USA

${ }^{144}$ University of Nebraska-Lincoln, Lincoln, Nebraska 68588, USA

${ }^{145}$ State University of New York at Buffalo, Buffalo, New York 14260, USA

${ }^{146}$ Northeastern University, Boston, Massachusetts 02115, USA

${ }^{147}$ Northwestern University, Evanston, Illinois 60208, USA

${ }^{148}$ University of Notre Dame, Notre Dame, Indiana 46556, USA

${ }^{149}$ The Ohio State University, Columbus, Ohio 43210, USA

${ }^{150}$ Princeton University, Princeton, New Jersey 08542, USA

${ }^{151}$ University of Puerto Rico, Mayaguez, Puerto Rico 00681, USA

${ }^{152}$ Purdue University, West Lafayette, Indiana 47907, USA

${ }^{153}$ Purdue University Calumet, Hammond, Indiana 46323, USA

${ }^{154}$ Rice University, Houston, Texas 77251, USA

${ }^{155}$ University of Rochester, Rochester, New York 14627, USA 
${ }^{156}$ The Rockefeller University, New York, New York 10021, USA

${ }^{157}$ Rutgers, The State University of New Jersey, Piscataway, New Jersey 08854, USA

${ }^{158}$ University of Tennessee, Knoxville, Tennessee 37996, USA

${ }^{159}$ Texas A\&M University, College Station, Texas 77843, USA

${ }^{160}$ Texas Tech University, Lubbock, Texas 79409, USA

${ }^{161}$ Vanderbilt University, Nashville, Tennessee 37235, USA

${ }^{162}$ University of Virginia, Charlottesville, Virginia 22904, USA

${ }^{163}$ Wayne State University, Detroit, Michigan 48202, USA

${ }^{164}$ University of Wisconsin, Madison, Wisconsin 53706, USA

${ }^{\mathrm{a}}$ Deceased.

${ }^{\mathrm{b}}$ Also at Vienna University of Technology, Vienna, Austria.

${ }^{\mathrm{c}}$ Also at CERN, European Organization for Nuclear Research, Geneva, Switzerland.

${ }^{\mathrm{d}}$ Also at Institut Pluridisciplinaire Hubert Curien, Université de Strasbourg, Université de Haute Alsace Mulhouse, CNRS/IN2P3, Strasbourg, France.

${ }^{\mathrm{e}}$ Also at National Institute of Chemical Physics and Biophysics, Tallinn, Estonia.

${ }^{\mathrm{f}}$ Also at Skobeltsyn Institute of Nuclear Physics, Lomonosov Moscow State University, Moscow, Russia.

${ }^{\mathrm{g}}$ Also at Universidade Estadual de Campinas, Campinas, Brazil.

${ }^{\mathrm{h}}$ Also at California Institute of Technology, Pasadena, USA.

${ }^{\mathrm{i}}$ Also at Laboratoire Leprince-Ringuet, Ecole Polytechnique, IN2P3-CNRS, Palaiseau, France.

${ }^{\mathrm{j}}$ Also at Joint Institute for Nuclear Research, Dubna, Russia.

${ }^{\mathrm{k}}$ Also at Suez University, Suez, Egypt.

${ }^{1}$ Also at British University in Egypt, Cairo, Egypt.

${ }^{\mathrm{m}}$ Also at Fayoum University, El-Fayoum, Egypt.

${ }^{\mathrm{n}}$ Also at Ain Shams University, Cairo, Egypt.

${ }^{\circ}$ Also at Université de Haute Alsace, Mulhouse, France.

${ }^{\mathrm{p}}$ Also at Brandenburg University of Technology, Cottbus, Germany.

${ }^{\mathrm{q}}$ Also at The University of Kansas, Lawrence, USA.

${ }^{\mathrm{r}}$ Also at Institute of Nuclear Research ATOMKI, Debrecen, Hungary.

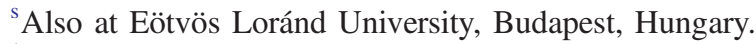

${ }^{\mathrm{t}}$ Also at University of Debrecen, Debrecen, Hungary.

${ }^{\mathrm{u}}$ Also at King Abdulaziz University, Jeddah, Saudi Arabia.

${ }^{\mathrm{v}}$ Also at University of Visva-Bharati, Santiniketan, India.

${ }^{\mathrm{w}}$ Also at University of Ruhuna, Matara, Sri Lanka.

${ }^{\mathrm{x}}$ Also at Isfahan University of Technology, Isfahan, Iran.

${ }^{y}$ Also at Sharif University of Technology, Tehran, Iran.

${ }^{\mathrm{z}}$ Also at Plasma Physics Research Center, Science and Research Branch, Islamic Azad University, Tehran, Iran.

${ }^{\text {aa } A l s o ~ a t ~ U n i v e r s i t a ̀ ~ d e g l i ~ S t u d i ~ d i ~ S i e n a, ~ S i e n a, ~ I t a l y . ~}$

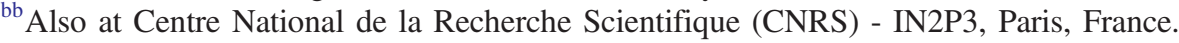

${ }^{c c}$ Also at Purdue University, West Lafayette, USA.

${ }^{\mathrm{dd}}$ Also at Universidad Michoacana de San Nicolas de Hidalgo, Morelia, Mexico.

${ }^{e e}$ Also at Institute for Nuclear Research, Moscow, Russia.

${ }^{\mathrm{ff}}$ Also at St. Petersburg State Polytechnical University, St. Petersburg, Russia.

${ }^{g g}$ Also at Faculty of Physics, University of Belgrade, Belgrade, Serbia.

${ }^{\text {hh }}$ Also at Facoltà Ingegneria, Università di Roma, Roma, Italy.

${ }^{\text {ii }}$ Also at Scuola Normale e Sezione dell'INFN, Pisa, Italy.

${ }^{\mathrm{jj}}$ Also at University of Athens, Athens, Greece.

${ }^{\mathrm{kk}}$ Also at Paul Scherrer Institut, Villigen, Switzerland.

${ }^{11}$ Also at Institute for Theoretical and Experimental Physics, Moscow, Russia.

${ }^{\mathrm{mm}}$ Also at Albert Einstein Center for Fundamental Physics, Bern, Switzerland.

${ }^{n n}$ Also at Gaziosmanpasa University, Tokat, Turkey.

${ }^{\circ o}$ Also at Adiyaman University, Adiyaman, Turkey.

${ }^{\mathrm{pp}}$ Also at Cag University, Mersin, Turkey.

${ }^{\mathrm{qq}}$ Also at Mersin University, Mersin, Turkey.

${ }^{\mathrm{rr}}$ Also at Izmir Institute of Technology, Izmir, Turkey.

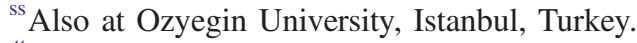

${ }^{\text {tt}}$ Also at Kafkas University, Kars, Turkey.

${ }^{\mathrm{uu}}$ Also at Mimar Sinan University, Istanbul, Istanbul, Turkey.

${ }^{\mathrm{vv}}$ Also at Rutherford Appleton Laboratory, Didcot, United Kingdom.

${ }^{w w}$ Also at School of Physics and Astronomy, University of Southampton, Southampton, United Kingdom. 
${ }^{\mathrm{xx}}$ Also at University of Belgrade, Faculty of Physics and Vinca Institute of Nuclear Sciences, Belgrade, Serbia.

${ }^{y y}$ Also at Argonne National Laboratory, Argonne, USA.

${ }^{z z}$ Also at Erzincan University, Erzincan, Turkey.

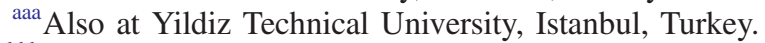

${ }^{b b b}$ Also at Texas A\&M University at Qatar, Doha, Qatar.

${ }^{\mathrm{ccc}}$ Also at Kyungpook National University, Daegu, Korea. 